\title{
17. MINERAL CHEMISTRY OF PRIMARY AND SECONDARY PHASES IN BASALTIC ROCKS, LEG 1291
}

\author{
G. Rowbotham ${ }^{2}$ and P. A. Floyd ${ }^{2}$
}

\begin{abstract}
Primary magmatic phases (spinel, olivine, plagioclase, clinopyroxene, amphibole, and biotite) and secondary phyllosilicates (smectite, chlorite-smectite, and celadonite) were analyzed by electron microprobe in alkalic and tholeiitic dolerites and basalts from Ocean Drilling Program Sites 800, 801, and 802. Aphyric alkalic dolerite sills (Hole 800A) and basalt flows (Holes 801B and $801 \mathrm{C}$ ) share common mineralogical features: matrix feldspars are strongly zoned from labradorite cores to discrete sodic rims of alkali feldspar with a high Or component, which overlaps that of quench microlites in glassy mesostasis; little fractionated clinopyroxenes are $\mathrm{Ti}$-rich diopsides and augites (with marked aegirine-augite rims at $\mathrm{Site} 801$ ); rare, brown, $\mathrm{Fe}^{3+}$-rich amphibole is winchite; and late biotites exhibit variable Ti contents. Alkalic rims to feldspars probably developed at the same time as quenched mesostasis feldspars and late-stage magmatic biotite, and represent the buildup of K-rich hydrous fluids during crystallization. Phenocryst phases in primitive mid-ocean ridge tholeiites from Hole $801 \mathrm{C}(\mathrm{Mg}$ numbers about 70 ) have extreme compositions with chrome spinel $(\mathrm{Cr} / \mathrm{Cr}+\mathrm{Al}$ ratios about $0.2-0.4)$, Ni-rich olivine $\left(\mathrm{Fo}_{90}\right)$, and highly calcic plagioclase $\left(\mathrm{An}_{90}\right)$. Later glomerophyric clumps of plagioclase $\left(\mathrm{An}_{75-80}\right)$ and clinopyroxene (diopside-augite) are strongly zoned and probably reflect rapidly changing melt conditions during upward transport, prior to seafloor quenching. In contrast, phenocryst phases (olivine, plagioclase, and clinopyroxene) in the Hole 802A tholeiites show limited variation and do not have such primitive compositions, reflecting the uniform and different chemical composition of all the bulk rocks.

Replacive phyllosilicates in both alkalic and tholeiitic basalts include various colored smectites ( $\mathrm{Fe}-, \mathrm{Mg}$-, and $\mathrm{Al}$-saponites), chlorite-smectite and celadonite. Smectite compositions typically reflect the replaced host composition: glass is replaced by brown $\mathrm{Fe}$-saponites (variable $\mathrm{Fe} / \mathrm{Mg}$ ratios) and olivine by greenish $\mathrm{Mg}$-saponites (or $\mathrm{Al}$-rich chlorite-smectite).
\end{abstract}

\section{INTRODUCTION}

Tholeiitic and alkalic basalts and dolerites were sampled from three sites during Ocean Drilling program (ODP) Leg 129 in the western Pacific Ocean (Fig. 1). This paper reports the chemistry of the dominant primary and secondary phases in selected basement samples in terms of magma type, petrogenesis, and the subsequent effects of secondary alteration.

The local acoustic basement at Site 800 in the northern Pigafetta Basin is composed of a series of massive alkalic dolerite sills characterized by high incompatible element contents and well-fractionated rare earth element (REE) patterns. They are comparable with wellevolved ocean island basalts (OIB) of predominantly hawaiite composition and probably represent deep-sea lateral offshoots from nearby seamounts of similar composition and age (Floyd et al., this volume). The best recovery of igneous basement was from Site 801 in the southern Pigafetta Basin, where the oldest segment of ocean crust in the Pacific Ocean, of Middle Jurassic age, was sampled (Lancelot, Larson, et al., 1990; Matsuoka, 1990) with radiometric ages ranging from about 157 to $167 \mathrm{Ma}$ (Pringle, this volume). The lava flows and pillow lavas of Site 801 comprise an upper alkalic basalt sequence separated from a lower tholeiitic basalt sequence by a distinctive yellow hydrothermal deposit (Floyd et al., this volume). The lower tholeitic sequence is mineralogically and chemically similar to incompatible element depleted basalts from normal mid-ocean ridge segments (N-MORB) and thus represents ocean crust of Jurassic age (Floyd et al., 1991). The alkalic basalt cap has affinities with typical OIB and probably represents off-axis volcanism rather than the last-stage product of highly fractionated axial seamounts. Drilling at Site 802 in the Mariana Basin penetrated acoustic basement, but

${ }^{\prime}$ Larson, R. L., Lancelot, Y., et al., 1992. Proc. ODP. Sci. Results, 129: College Station, TX (Ocean Drilling Program).

${ }^{2}$ Department of Geology, University of Keele, Staffordshire, ST5 5BG, United Kingdom.

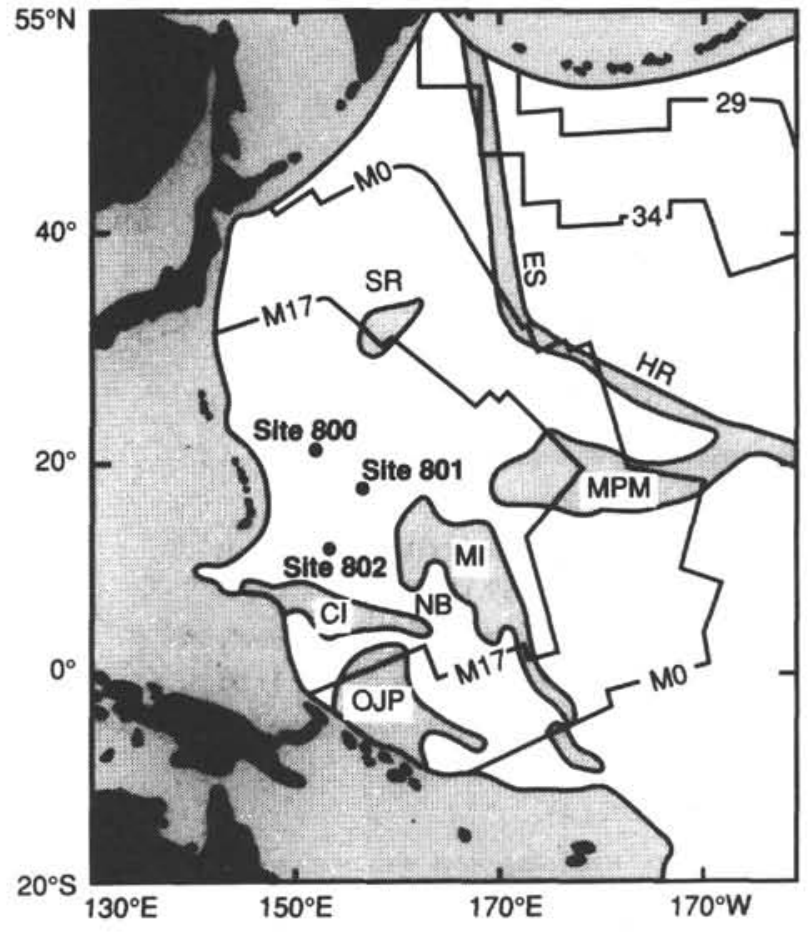

Figure 1. Map of the western Pacific Ocean showing the position of Leg 129 Sites 800, 801, and 802 (Lancelot, Larson, et al., 1990). The unshaded area represents normal Pacific Ocean crust with magnetic lineation contours, whereas shaded areas represent thickened crustal sections and younger crust to the west of the Pacific subduction zones. Feature abbreviations are as follows: Caroline Islands (CI), Ontong-Java Plateau (OJP), Marshall Islands (MI), Nauru Basin (NB), Mid-Pacific Mountains (MPM), Shatsky Rise (SR), Hawaiian Ridge (HR), and Emperor Seamounts (ES). 
the composition of tholeiitic pillow lavas of Cretaceous age (about $115 \mathrm{Ma}$; Pringle, this volume) was considered to represent an extrusive event postdating the local underlying basement of Jurassic age (Lancelot, Larson, et al., 1990). Relative to N-MORB, the predominantly olivine-plagioclase-clinopyroxene phyric tholeiites are enriched in large-ion-lithophile (LIL) elements with flat, unfractionated REE patterns, and exhibit compositions similar to those of ocean plateau basalts (Floyd, 1989) of similar age in the western Pacific.

In summary, the basic rocks of Sites 800 and 802 are representative of the widespread mid-Cretaceous volcanic event in the western Pacific (Lancelot, Larson, et al., 1990), with the former composed of alkalic dolerite sills and the latter of submarine tholeiitic basalts. Site 801 , on the other hand, is a section through oceanic crust of Jurassic age and is composed of alkalic and tholeiitic sequences.

\section{ANALYTICAL TECHNIQUES}

Analyses of all mineral phases were obtained using both Cameca (Camebax) and Cambridge Instruments Geoscan electron microprobes in the Department of Geology, University of Manchester. The operating conditions were $15 \mathrm{kV}$ and $3.5 \times 10^{-8} \mathrm{~A}$ and a spot size of about $3 \mu \mathrm{m}$, except in the analysis of hydrous phases, when the beam was defocused. Metals, oxides, and silicate materials were used in calibration; an estimate of the accuracy and precision of the technique employed is given by Dunham and Wilkinson (1978), with corrections using standard software program ZAF4-FLS from Link Analytical. $\mathrm{Fe}^{3+}$ values were determined via stoichiometry after the method of Droop (1987) and with reference to the limitations of the estimates (Schumacher, 1991). Olivine analyses, while in equilibrium with the adjacent glass, contain variable $\mathrm{Na}$ and $\mathrm{Al}$ contents that probably reflect some contamination by secondary replacement minerals. Determination and calculation of carbonate end-members can only be considered estimates.

\section{PETROGRAPHY OF ANALYZED SAMPLES}

Mineral phases analyzed by microprobe in basalt and dolerite samples from Holes $800 \mathrm{~A}, 801 \mathrm{~B}, 801 \mathrm{C}$, and $802 \mathrm{~A}$ were selected to show as complete a range of primary and secondary mineral compositions as possible. A checklist of the phases analyzed in different rock samples is given in Table 1 and the corresponding bulk-rock geochemistry is presented elsewhere in this volume (Floyd and Castillo; Floyd et al.). The general petrography of each bulk-rock sample from the different holes is outlined in the following.

\section{Hole 800A}

Both of the samples selected for phase analysis (129-800A-58R-1, $86-91 \mathrm{~cm}$, and $800 \mathrm{~A}-58 \mathrm{R}-2,67-73 \mathrm{~cm}$ ) are from cooling unit 3 of the alkalic dolerites and are well evolved mineralogically and chemically. They have a similar primary petrography, although in Sample 800 A-58R-2, 67-73 cm, the secondary phases are better developed. Olivine is not present in this cooling unit.

In both samples the plagioclase laths (up to $2 \mathrm{~mm}$ long) exhibit an oscillatory zoning, although there is a sharp optical discontinuity between the core and rim portions of many individual crystals. Albite twinning, characteristic of the plagioclase cores, is not present at the margins and suggests that the more sodic rims represent a later overgrowth, which may in some cases be intergrown with mafic minerals. Most feldspars are relatively fresh, although the cores are invariably replaced by secondary opaque material. Mafic minerals are pink pleochroic clinopyroxene, a pale brown amphibole, and minor biotite, of which the latter may be largely secondary in origin. Clinopyroxene varies widely in shape and size, and is commonly replaced by a pale green smectite. The amphibole either replaces clinopyroxene or occurs as discrete ragged subhedral crystals and is clearly later in development than either plagioclase or clinopyroxene. Biotite commonly fringes titanomagnetite grains and needles or replaces clays in earlier replacement domains. Much of the biotite growth may have been in response to subsequent contact metamorphism by adjacent sills (Floyd et al., this volume). Rare, euhedral pseudohexagonal basal sections of biotite exhibit a color zonation showing dark brown rims, as well as partial altered to a green phyllosilicate. Apatite needles are abundant.

The nucleation sites of secondary phyllosilicates are grain boundaries and along cleavages, but are particularly well-developed in

Table 1. Mineral phases analyzed (+) in selected samples of dolerites and basalts from Leg 129.

\begin{tabular}{|c|c|c|c|c|c|c|c|c|}
\hline $\begin{array}{l}\text { Core, section, } \\
\text { interval }(\mathrm{cm})\end{array}$ & Feldspar & Pyroxene & Olivine & Spinel & Biotite & Amphibole & Smectite & Celadonite \\
\hline \multicolumn{9}{|l|}{$129-800 \mathrm{~A}-$} \\
\hline $58 \mathrm{R}-1,86-91$ & + & + & & & + & + & + & \\
\hline $58 \mathrm{R}-2,67-73$ & + & + & & & + & + & + & \\
\hline \multicolumn{9}{|l|}{ 129-801B- } \\
\hline $41 \mathrm{R}-1,130-136$ & + & + & & & + & & + & \\
\hline $43 \mathrm{R}-1,4-9$ & + & + & & & + & & + & \\
\hline $44 R-2,140-145$ & + & + & & & + & & + & \\
\hline $44 \mathrm{R}-3,28-33$ & + & & & + & & & & \\
\hline \multicolumn{9}{|l|}{$129-801 \mathrm{C}-$} \\
\hline IR-1, 14-20 & + & + & & & + & & + & \\
\hline $2 R-5,101-106$ & + & + & & & + & 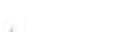 & + & + \\
\hline $5 R-2,123-130$ & + & & & & & & + & + \\
\hline $5 R-3,125-131$ & + & & & & & & + & + \\
\hline $6 R-4,49-54$ & + & + & + & + & & & + & \\
\hline $7 R-2,122-127$ & + & + & & & & & & + \\
\hline $9 \mathrm{R}-5,0-7$ & + & + & & & & & + & + \\
\hline \multicolumn{9}{|l|}{$129-802 \mathrm{~A}-$} \\
\hline $58 \mathrm{R}-1.96-100$ & + & + & & & & & + & \\
\hline $58 \mathrm{R}-1,99-102$ & + & + & & & & & + & + \\
\hline $58 \mathrm{R}-3,90-95$ & + & + & + & & & & + & \\
\hline $59 \mathrm{R}-1,138-142$ & + & + & & & & & + & + \\
\hline $59 \mathrm{R}-2,40-44$ & + & + & & & & & + & + \\
\hline $62 \mathrm{R}-2,53-59$ & + & + & & & & & + & \\
\hline
\end{tabular}


primary mafic silicates and interstitial areas, where they form smectite-rich alteration domains. Most smectites are fibrous and green in color, although the more granular-looking varieties are invariably brownish. At the boundary between primary and secondary phases, phyllosilicates are commonly a dark green color, which is replaced outward by birefringent pale green fibers $(0.05-0.075 \mathrm{~mm}$ long), which are intimately associated with late replacive carbonate.

\section{Site 801}

\section{Alkalic Basalts and Microdolerites}

The alkalic rocks of Site 801 are distinct from the alkalic dolerites of Hole $800 \mathrm{~A}$ in being generally finer-grained throughout (basalts and microdolerites), extrusive thin flows rather than sills (with quenchedtextured matrices and glassy mesostasis in the microdolerites), and olivine bearing (as phenocrysts and in the groundmass). They are generally aphyric, although some plagioclase-olivine phyric basalts occur toward the base of Hole 801B. On chemical grounds two groups of alkalic basalts can be recognized (Floyd and Castillo, this volume): a primitive olivine-rich group and a more evolved, olivine-poor group that contains some plagioclase-olivine phyric basalts. The dominant flows are intruded by a minor plagioclase-olivine phyric quenched basalt of similar mineralogy to the more evolved phyric flows.

The main petrographic differences between the olivine-rich alkalic basalts (represented by Samples 801B-43R-1,4-9 cm, and 801C-1R-1, $14-20 \mathrm{~cm}$ ) and the remainder is the proportion of olivine and the presence of green rims to the pinkish clinopyroxenes, minor brown amphibole, and slightly less titanomagnetite; otherwise, they are mineralogically and texturally similar. The rest of the samples used for probe work (Table 1) show similar features, apart from differences in texture, as described in the following. Olivine is always pseudomorphed by greenish smectites but is generally recognizable by its characteristic euhedral form. Apart from zoned phenocrysts, there are two generations of plagioclase in the matrix: large (maximum length of up to $3 \mathrm{~mm}$ ), interlocking fresh laths, which are invariably optically zoned and constitute the main phase, and a later, second generation of curved and radiating microlites (up to $0.5 \mathrm{~mm}$ long) developed in the quenched mesostasis. Other minerals in the mesostasis consist of an admixture of clay minerals replacing original glass, titanomagnetite granules, biotite flakes, and titanite. These features are well displayed in Samples 801B-41R-1, 130-136 cm, 801B-43R-1, 4-9 cm, and 801B-44R-2, $140-145 \mathrm{~cm}$. In the coarser flow interiors the euhedral to subhedral clinopyroxenes are lilac to brown in color (up to $5 \mathrm{~mm}$ in length) with a faint pleochroism and optically zoned. They are commonly altered along the cleavages and around the rims to a pale green smectite. Pseudohexagonal plates of dark brown, strongly pleochroic biotite are common as a late primary phase (up to $0.8 \mathrm{~mm}$ diameter) and are found rimming earlier titanomagnetite (for example, Sample 801B-41R-1, $130-136 \mathrm{~cm}$ ). Common accessory phases are skeletal titanomagnetite with a high length-to-width ratio (20:1) and acicular needles of apatite up to $0.6 \mathrm{~mm}$ in length.

Superimposed upon this primary assemblage is a secondary mineralogy dominated by pale green and brownish smectites. In particular they form radiating fibrous patches within the mesostasis of the microdolerites or throughout the matrix of the quenched basalts. Phyllosilicates also nucleate on the margins of primary silicates such as clinopyroxene. The occurrence of a number of generations of smectite growth has also been recorded, with light green radiating crystals apparently representing the recrystallization of pre-existing fine-grained phyllosilicates.

\section{Tholeiitic Basalts}

The tholeiitic rocks below the hydrothermal deposit in Hole $801 \mathrm{C}$ are divided into two petrographic-chemical groups: (1) an upper sequence (Cores $801 \mathrm{C}-1 \mathrm{R}$ to $801 \mathrm{C}-6 \mathrm{R}$, encompassing cooling units C9-C20) of chemically primitive olivine-plagioclase-spinel phyric basalts and (2) a lower sequence (Cores $801 \mathrm{C}-7 \mathrm{R}$ to $801 \mathrm{C}-12 \mathrm{R}$, encompassing cooling units $\mathrm{C} 21-\mathrm{C} 32$ ) of chemically evolved (generally) aphyric, vesicular basalts (Floyd and Castillo, this volume). Compared with the majority of selected samples, those from the upper sequence have been extensively altered by circulating hydrothermal fluids with the subsequent deposition of various green clays and carbonate (especially Samples $801 \mathrm{C}-5 \mathrm{R}-2,123-130 \mathrm{~cm}$, and $801 \mathrm{C}$ $5 R-3,125-131 \mathrm{~cm}$ ).

The primitive phyric basalts are quench-textured throughout, with samples selected for analysis from glassy flow rims (for example, Sample $801 \mathrm{C}-5 \mathrm{R}-2,123-130 \mathrm{~cm}$ ) to variolitic and intersertal-textured flow interiors (for example, Sample 129-801C-5R-5, 73-78 $\mathrm{cm})$. In most samples plagioclase is relatively fresh and forms both megaphenocrysts $(4 \times 3 \mathrm{~mm})$ and either serrated laths in flow interiors or forked microlites in the glassy rims. In Sample 801C$6 \mathrm{R}-4,49-54 \mathrm{~cm}$, however, plagioclase forms zoned glomerocrysts $(2.5 \times 2.5 \mathrm{~mm})$ that often contain rounded inclusions of glass (original melt) and small spinels and olivines. There is also a second generation of plagioclase microphenocrysts $(0.6 \times 0.25 \mathrm{~mm})$ that also contain spinel octahedra, but no glass inclusions. Olivine (invariably pseudomorphed by smectites) occurs as euhedral microphenocrysts or small grains scattered throughout the matrix. In some samples (for example, $801 \mathrm{C}-6 \mathrm{R}-4,49-54 \mathrm{~cm})$ relatively large olivine phenocrysts $(0.8 \times 3.0 \mathrm{~mm})$ contain minute spinels and subrounded inclusions of a quenched dendritic phase. Spinel occurs in two generations: dark brown microphenocrysts commonly loosely associated with olivine in the matrix or minute, pale yellow-brown octahedra within plagioclase and olivine phenocrysts (for example, Samples 801C-5R-5, 73-78, and 801C-6R-4, 49-54 cm). Clinopyroxene is the only major silicate non-phenocrystic phase, but can form two associated textural variants in the coarser flow interiorsvariolitic fans, with or without coaxial plagioclase, and groups of interspersed granular crystals. Coarser clinopyroxene grains may be optically zoned with hour-glass structure or early crystallizing subophitic groups passing outward into quenched variolitic growths. Although opaque oxides are generally partly obscured by dark brown smectitic material, in reflected light they are skeletal in outline.

The two samples of the evolved chemical group (Samples 801C$7 \mathrm{R}-2,122-127 \mathrm{~cm}$, and $801 \mathrm{C}-9 \mathrm{R}-5,0-7 \mathrm{~cm}$ ) are both slightly phyric with scattered plagioclase glomerocrysts, none of which contains inclusions of glass or early primary phases, like the primitive group samples described previously. The outer parts of the flows are characterized by curved and straight plagioclase microlites typically acting as nucleation sites for spherulites. Flow interiors are typically intersertal and variolitic, again exhibiting the variation in clinopyroxene morphology noted previously.

All samples selected for probe work have been affected by alteration with the development of various smectites, celadonite, carbonate, pyrite, and rare zeolites. Phyllosilicates were analyzed from a number of different locations, including zoned vesicles, olivines and coarse vein material. For example, in the chilled margin of Sample 801C-5R$2,123-130 \mathrm{~cm}$ are mineralogically zoned vesicles with walls lined initially with brown and subsequently various bright green clays. Veins contain green smectites and coarser grained colorless birefringent phyllosilicates, together with late replacive carbonate. The habit of the secondary minerals varies from fine-grained fibers that nucleate on a precursor phase to large plates, which are late-stage vein infillings.

\section{Hole 802A}

The tholeiitic basalts selected from Hole $802 \mathrm{~A}$ are predominantly phyric with olivine, plagioclase, and clinopyroxene phenocrysts, commonly developed in medium-grained glomerophyric groups. Textural variability throughout the flows is largely related to cooling history, ranging from fresh glassy rims (Sample 802A-58R-2, 99-102 cm) and a spherulitic or variolitic zone to flow interiors with an intersertal texture and subophitic growth centers (Sample 802A-62R-2, 53-59 cm). 
Plagioclase forms two generations of phenocrysts ranging from large (about $1 \mathrm{~mm}$ ), magmatically corroded, euhedral crystals to smaller laths associated with clinopyroxene in glomerophyric clumps. Both of these early generations have either oscillatory, normal, or reverse zoning with sharp optical discontinuities between core and rim. The latest generation of plagioclase represents matrix and is composed of serrated laths that are intimately associated with clinopyroxene in variolitic growths (for example, Samples 802A-59R-1, 138-142 cm and $802 \mathrm{~A}-59 \mathrm{R}-3,40-44 \mathrm{~cm}$ ). Clinopyroxene may also form three growth generations: early subhedral glomerocrysts associated with plagioclase laths, granular aggregates dispersed between quenched sheaf variolites composed of radiate clinopyroxene. In coarser grained flow interiors (for example, Sample 802A-62R-2, 53-59 cm) the texture may be sub-ophitic with elongate plagioclase laths (up to $1 \mathrm{~mm}$ long). Olivine always occurs as (smectite-replaced) microphenocrysts scattered throughout both glassy rims and variolitic flow interiors.

Fresh glass preserved at flow margins is rare, but may exhibit all three phenocryst phases (olivine, plagioclase, and clinopyroxene) that are commonly used as nucleation centers for dark spherulites (for example, Sample 802A-58R-2, 99-102 cm). Away from the glassy spherulitic rind the texture is variolitic, but the phenocryst phases have similar dimensions to those in the glass: olivine $(0.5 \mathrm{~mm})$, clinopyroxene $(0.5 \mathrm{~mm})$, and plagioclase $(0.75-0.80 \mathrm{~mm})$.

Secondary alteration is confined to smectites, celadonite, pyrite, and carbonate. Secondary assemblages are well-developed in Sample 802A58R-2, 99-102 cm, where alteration domains ( $>5 \mathrm{~mm}$ diameter) consist of late carbonate replacing green and brown phyllosilicates in the matrix. The olivines are always pseudomorphed by brown smectite that initially developed along original fractures before replacing the whole phenocryst.

\section{PRIMARY MINERAL PHASE CHEMISTRY}

Because of the marked chemical differences and compositional range exhibited by alkalic and tholeiitic basic rocks, primary magmatic phases in the alkalic rocks (Hole 800Adolerites and Holes 801B and $801 \mathrm{C}$ basalts) will be described first, followed by the tholeiitic basic rocks (Hole $801 \mathrm{C}$ basalts and Hole $802 \mathrm{~A}$ basalts). Fresh glass is relatively rare throughout Leg 129 basalts and although a few analyses are recorded here (see Table 10) no comment has been made at this stage on their composition or petrogenesis.

\section{Alkalic Dolerites and Basalts}

\section{Feldspars}

The main petrographic characteristics of the feldspars (Table 2) in both the alkali dolerites and basalts are the presence of normal and oscillatory zoning within the albite twinned portions of plagioclase laths and a lack of multiple twinning in the outmost zones. Many matrix feldspars (for example, Samples 801B-43R-1, 4-9 cm; 801B-44R-2, $140-145 \mathrm{~cm}$, and $801 \mathrm{C}-1 \mathrm{R}-1,14-20 \mathrm{~cm}$ ) exhibit sharp chemical discontinuities between the highly calcic cores and twin-free sodic rims, whereas other crystals show almost continuous zoning. The most calcic cores of plagioclases from Hole $800 \mathrm{~A}$ alkaline dolerites (Samples 800A-58R-2, 67-73 cm and 800A-58R-1, 86-91 cm) are sodic labradorites $\left(\mathrm{An}_{55-53} \mathrm{Ab}_{44-40} \mathrm{Or}_{1-7}\right)$ (Fig. 2) and are about $15 \%$ less anorthitic than plagioclase cores from Hole $801 \mathrm{C}$ alkali basalts, which are typically labradorites/bytownites (for example, $\mathrm{An}_{69} \mathrm{Ab}_{29} \mathrm{Or}_{2}$ in Sample 801C-43R-1,4-9 cm). As seen in Figure 2, the compositional trends of the plagioclase feldspars are all towards enrichment in the albite component, although there are differences in detail whereby the dolerite plagioclases have systematically lower Or contents at the sodic end than the plagioclases from the basalts. For example, a sodic plagioclase composition of $\mathrm{An}_{16} \mathrm{Ab}_{68} \mathrm{Or}_{26}$ from Hole 801B basalts (Sample 801B-44R-2, 140-145 cm) is about 20\% richer in Or than a corresponding plagioclase in the dolerites. All further sodic compositions then change toward the alkali feldspars (Fig. 2). The range of compositions is identical in all of the feldspars from Holes $801 \mathrm{~B}$ and $801 \mathrm{C}$.

Most of the analyzed grains showed normal zoning (with calcic cores and sodic rims), but oscillatory zoning is not uncommon. For example, a plagioclase from Sample 800A-58R-2, 67-73 cm demonstrates the changes in composition in adjacent zones outward: $\mathrm{An}_{56} \mathrm{Ab}_{43} \mathrm{Or}_{0}$ (labradorite core); $\mathrm{An}_{31} \mathrm{Ab}_{67} \mathrm{Or}_{2} ; \mathrm{An}_{39} \mathrm{Ab}_{59} \mathrm{Or}_{2} ; \mathrm{An}_{18} \mathrm{Ab}_{76} \mathrm{Or}_{6} ; \mathrm{An}_{2} \mathrm{Ab}_{63} \mathrm{Or}_{35}$ (alkali feldspar rim). These compositional changes are quite large and must reflect rapid changes in the liquid from which these feldspars crystallized. As illustrated in the preceding case, most of the rims of the Hole $800 \mathrm{~A}$ feldspar laths are true alkali feldspars. The width of the alkali feldspar zone in the crystals varies from the extreme rim to occupying a large proportion of the feldspar lath itself. Alkali feldspar rims are also common in the basalts from Holes 801B and 801C. The most extreme alkali feldspar compositions encountered were $\mathrm{An}_{0} \mathrm{Ab}_{31} \mathrm{Or}_{69}$ in an alkalic basalt (Hole 801B) and $\mathrm{An}_{2} \mathrm{Ab}_{45} \mathrm{Or}_{54}$ in an alkalic dolerite (Hole 800A). No reversed zoning has been noted in these new compositions.

Quench crystals of alkali feldspars also occur in alkalic basalt mesostasis (Site 801) and have compositions that (1) completely overlap those of the rims of the matrix laths and (2) show little systematic chemical variation across or along individual alkali feldspar microlites, for example, $\mathrm{An}_{0} \mathrm{Ab}_{43} \mathrm{Or}_{57}$ (at growth point); $\mathrm{An}_{3} \mathrm{Ab}_{61} \mathrm{Or}_{36} ; \mathrm{An}_{2} \mathrm{Ab}_{56} \mathrm{Or}_{42} ; \mathrm{An}_{3} \mathrm{Ab}_{63} \mathrm{Or}_{34}$ (to extreme tip).

One alkali basalt analyzed (Sample $801 \mathrm{~B}-44 \mathrm{R}-3,28-33 \mathrm{~cm}$ ) is texturally different and contains both phenocrystic and groundmass plagioclase. The phenocrysts again exhibit the continuous zoning of the cores with sharp transitions to alkali feldspar rims; for example, $\mathrm{An}_{69} \mathrm{Ab}_{29} \mathrm{Or}_{2}$ (core) to $\mathrm{An}_{5} \mathrm{Ab}_{62} \mathrm{Or}_{33}$ (rim). The groundmass plagioclase do not show such pronounced alkalic rims and have an average composition of about $\mathrm{An}_{56} \mathrm{Ab}_{40} \mathrm{Or}_{4}$. The main minor elements in these feldspars are $\mathrm{Fe}$ and $\mathrm{Mg}$, but their concentrations are quite low, almost exclusively below 0.50 cations per formula unit (cfu).

Overall the feldspars analyzed in both the Hole $800 \mathrm{~A}$ dolerites and Site 801 basalts have similar textural and chemical characteristics to those described by Upton et al. (1985) from the Tugtutoq Older Giant Dyke Complex in south Greenland. They also exhibit a unidirectional variation trend (as shown by the Group 1 feldspars described by Henderson and Gibb, 1983), ranging from plagioclase to Or-rich compositions, with no indication of convergence of two compositionally distinct feldspars. The alkali feldspar rims of both the Greenland samples and those of the present study extend beyond the projected position of the experimental $5 \mathrm{~kb}$ cotectic $\left(\mathrm{Ab}_{50} \mathrm{Or}_{50}\right)$ - a feature that has been interpreted as due to the presence of an additional phase, nepheline (Upton et al., 1985). Although the alkalic rocks of Leg 129 are nepheline-normative (Floyd and Castillo, this volume) no modal nepheline has been observed, including the most evolved samples. This may suggest that the true position of the cotectic is closer to the Or apex than indicated by experimental systems. The other significant feature is that the alkalic rims to the feldspars probably developed at the same time as the abundant biotite and represent the buildup of late aqueous potassic fluids during the final stages of alkalic melt crystallization.

\section{Pyroxenes}

The composition of the pyroxenes (Tables 3 and 4) from the alkaline dolerite sills (Hole 800A) straddles the boundary between diopside and augite (Morimoto, 1988) and is typical of clinopyroxenes developed in alkalic sills and dikes (Wilkinson, 1956; Henderson and Gibb, 1988). They show little systematic variation in terms of pyroxene quadrilateral components (Fig. 3) with the maximum compositional variation represented by a zoned crystal from Sample 800A-58R-1, 86-91 cm, with a core composition of $\mathrm{Ca}_{44} \mathrm{Mg}_{42} \mathrm{Fe}_{14}$ and rim of $\mathrm{Ca}_{42} \mathrm{Mg}_{40} \mathrm{Fe}_{18}$. In contrast, clinopyroxenes from the alkali basalts (Holes 801B and 801C) are all diopsides (Fig. 3), although once again little fractionation is observed between uniform core compositions (for example, $\mathrm{Ca}_{50} \mathrm{Mg}_{33} \mathrm{Fe}_{17}$ ) and slightly Fe-enriched 


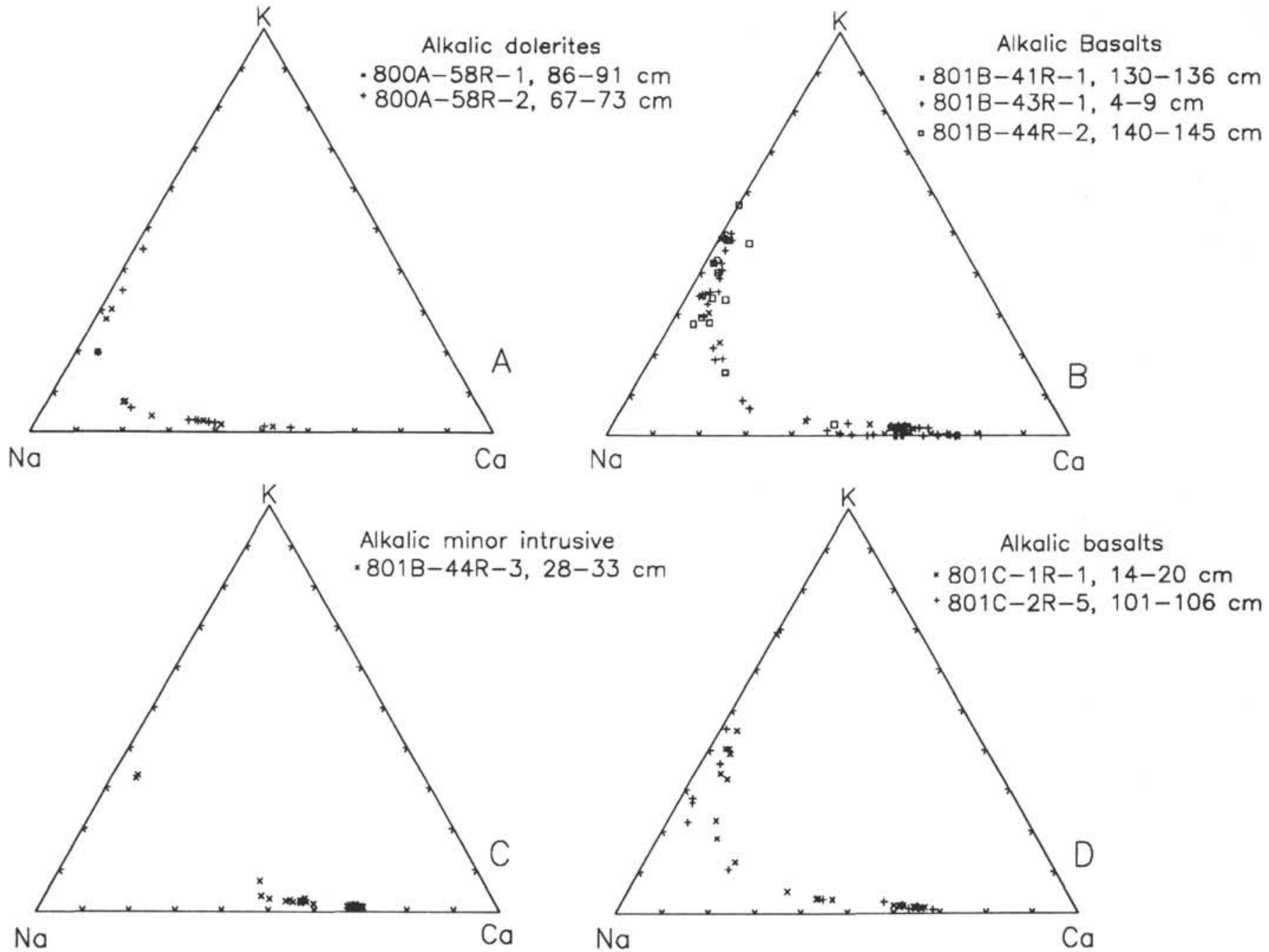

Figure 2. Composition of feldspars from alkalic dolerites and basalts, in terms of orthoclase (K), albite (Na) and anorthite (Ca) components. A. Dolerite sills from Hole 800A. B. Basalt flows from Hole 801B. C. Minor basalt intrusive from Hole 801C. D. Basalt flows from Hole 801C.

rims. These calculations have been made on the basis that $\mathrm{Ca}^{2+}$ substitutions are within the field of the quadrilateral components and exclude the small component of a possible Tschermak's substitution. The ferric iron content of the diopsidic clinopyroxenes (Site 801) has been calculated for a limited number of analyses and is quite variable $\left(0.04-0.14 \mathrm{Fe}^{3+} \mathrm{cfu}\right)$. There is, however, little systematic increase or decrease between core and rim.

The dominant minor constituents in all the alkalic clinopyroxenes analyzed are $\mathrm{Al}$ and $\mathrm{Ti}$. The maximum value of $\mathrm{Ti}$ is $0.07 \mathrm{cfu}$ and $\mathrm{Al}$ $0.25 \mathrm{cfu}$. Both these elements are positively correlated in the clinopyroxenes with Ti:Al ratios between 1:2 and 1:4 (Fig. 4). Tracy and Robinson (1977) suggested that there was a relationship between the entry of $\mathrm{Ti}^{4+}$ into the pyroxene lattice and substitutions in both the octahedral and tetrahedral sites of the type: $\mathrm{Fe}^{2+}+\mathrm{Ti}^{4+}+2 \mathrm{Al}^{3+}=2 \mathrm{Mg}^{2+}$ $+2 \mathrm{Si}^{4+}$. The substitution is common within alkali basalt sills in general (Robinson, 1980; Henderson and Gibb, 1988); the validity of this substitution has been supported experimentally by Sack and Carmichael (1984). A similar relationship is also recorded in Leg 129 intrusive and extrusive alkalic rocks. If this were the sole coupled substitution involving $\mathrm{Al}^{3+}$ in the clinopyroxenes, the ratio $\mathrm{Ti}^{4+} / \mathrm{Al}^{3+}$ would be 1:2, whereas it varies to below 1:4 (Fig. 4) and suggests that the excess $\mathrm{Al}^{3+}$ occurs in the limited substitution of Tschermak's molecule $\left(\mathrm{CaAlAlSiO}_{6}\right)$. Other nonquadrilateral components such as $\mathrm{Na}^{+}(<0.07 \mathrm{cfu})$ and $\mathrm{K}^{+}(<0.01 \mathrm{cfu})$ are extremely low.
In addition to the dominant diopsidic clinopyroxenes of Site 801 there are some with strongly alkalic rims. Alkali pyroxenes were analyzed (Table 4) in three Samples (801B-43R-1, 4-9 cm, 801B-44R$2,140-145 \mathrm{~cm}$ and $801 \mathrm{C}-1 \mathrm{R}-1,14-20 \mathrm{~cm}$ ) and in all cases showed a sharp chemical discontinuity between the diopside core (for example, $\mathrm{Di}_{54} \mathrm{Hd}_{38} \mathrm{Ac}_{8}$ ) and the alkali pyroxene rim (for example, $\mathrm{Di}_{25} \mathrm{Hd}_{48} \mathrm{Ac}_{27}$ ) (Fig. 5). The alkali pyroxenes zone outward to some highly aegirinerich compositions, such as $\mathrm{Di}_{8} \mathrm{Hd}_{10} \mathrm{Ac}_{82}$ (Samples 801B-43R-1, 4-9 $\mathrm{cm}$ and $801 \mathrm{~B}-44 \mathrm{R}-2,140-145 \mathrm{~cm}$ ). In addition, the alkali pyroxenes are all highly titaniferous (up to $0.15 \mathrm{cfu}$ ) and are classified as titanian aegirine-augites and titanian augites (Morimoto, 1988). Naturally occurring highly titaniferous alkali pyroxenes have been described from peralkaline volcanic and plutonic rocks (Schmincke, 1969; Ferguson, 1977; Curtis and Gittins, 1979) where they are commonly found as a late-stage magmatic phase after Ti-poor aegirine-augite and growing discontinuously on diopsidic pyroxenes. Laverne (1987) has also reported occurrences in veins cross-cutting oceanic basalts (Hole $504 \mathrm{~B}$ ), but the compositions are more diverse than those reported here and include aegirine-augite and fassaite. The main difference between the data presented by Laverne (1987) and the present study is that Leg 129 alkali pyroxenes exhibit a much more extended solid solution toward the aegirine end-member composition and also little jadeite substitution, that is, no $\mathrm{Al}^{3+}$ in octahedral coordination sites (Table 4). The entry of $\mathrm{Ti}^{4+}$ into the octahedral sites of these clinopy- 
G. ROWBOTHAM, P. A. FLOYD

Table 2. Feldspar analyses from alkalic dolerites and basalts, Holes 800A, 801B, and $801 \mathrm{C}$.

\begin{tabular}{|c|c|c|c|c|c|c|c|c|c|c|c|}
\hline Hole & $800 \mathrm{~A}-$ & $800 \mathrm{~A}-$ & $800 \mathrm{~A}-$ & 800A. & $800 \mathrm{~A}-$ & $800 \mathrm{~A}$ - & 801B- & 801B - & 801B- & 801B- & 801B- \\
\hline Core/section & $58 R-1$ & $58 R-1$ & $58 R-1$ & $58 R-2$ & $58 R-2$ & 58R-2. & $43 R-1$ & $43 R-1$ & $43 R-1$ & $43 R-1$ & 44R-3. \\
\hline Interval $(\mathrm{cm})$ & $86-91$ & $86-91$ & $86-91$ & 67.73 & $67-73$ & $67-73$ & $4-9$ & $4-9$ & $4-9$ & $4-9$ & $28-33$ \\
\hline & C & C & $\mathbf{R}$ & C & $R / C$ & $\mathbf{R}$ & G & c & $R / C$ & $\mathbf{R}$ & c \\
\hline $\mathrm{SiO}_{2}$ & 64.85 & 60.73 & 62.43 & 54.88 & 63.02 & 67.23 & 66.86 & 51.73 & 60.86 & 65.62 & 51.32 \\
\hline $\mathrm{TiO}_{2}$ & 0.25 & 0.10 & 0.21 & 0.13 & 0.24 & 0.00 & 0.06 & 0.15 & 0.24 & 0.09 & 0.08 \\
\hline $\mathrm{Al}_{2} \mathrm{O}_{3}$ & 22.13 & 23.96 & 23.23 & 27.80 & 21.48 & 17.87 & 19.04 & 29.88 & 23.48 & 19.44 & 30.74 \\
\hline $\mathrm{FeO}$ & 0.12 & 0.35 & 0.22 & 0.61 & 0.24 & 0.86 & 0.29 & 0.48 & 0.46 & 0.28 & 0.50 \\
\hline MnO & 0.00 & 0.09 & 0.00 & 0.00 & 0.06 & 0.00 & 0.05 & 0.00 & 0.01 & 0.00 & 0.00 \\
\hline $\mathrm{MgO}$ & 0.12 & 0.00 & 0.02 & 0.00 & 0.00 & 0.06 & 0.00 & 0.02 & 0.00 & 0.00 & 0.00 \\
\hline $\mathrm{CaO}$ & 3.46 & 6.22 & 5.02 & 11.28 & 3.60 & 0.06 & 0.36 & 13.57 & 5.57 & 1.02 & 14.41 \\
\hline $\mathrm{Na}_{2} \mathrm{O}$ & 8.89 & 7.64 & 8.23 & 4.84 & 8.47 & 6.57 & 5.60 & 3.55 & 7.38 & 6.58 & 3.31 \\
\hline $\mathrm{K}_{2} \mathrm{O}$ & 1.34 & 0.61 & 0.68 & 0.20 & 1.11 & 7.17 & 8.25 & 0.33 & 1.17 & 6.89 & 0.23 \\
\hline $\mathrm{Cr}_{2} \mathrm{O}_{3}$ & 0.11 & 0.03 & 0.03 & 0.03 & 0.00 & 0.02 & 0.00 & 0.00 & 0.00 & 0.09 & 0.00 \\
\hline NiO & 0.00 & 0.00 & 0.00 & 0.10 & 0.00 & 0.00 & 0.09 & 0.00 & 0.40 & 0.22 & 0.00 \\
\hline 0 & 0.00 & 0.00 & 0.00 & 0.00 & 0.00 & 0.00 & 0.00 & 0.00 & 0.00 & 0.00 & 0.00 \\
\hline TOTAL\% & 101.26 & 99.73 & 100.09 & 99.87 & 98.21 & 99.86 & 100.59 & 99.70 & 99.57 & 100.21 & 100.59 \\
\hline Recalculation & to $8(0)$ & & & & & & & & & & \\
\hline si & 2.84 & 2.72 & 2.77 & 2.49 & 2.84 & 3.02 & 2.99 & 2.36 & 2.73 & 2.95 & 2.33 \\
\hline $\mathrm{Ti}$ & 0.01 & 0.00 & 0.01 & 0.01 & 0.01 & 0.00 & 0.00 & 0.01 & 0.01 & 0.00 & 0.00 \\
\hline$A l$ & 1.14 & 1.26 & 1.22 & 1.48 & 1.14 & 0.95 & 1.00 & 1.61 & 1.24 & 1.03 & 1.64 \\
\hline $\mathrm{Fe}$ & 0.00 & 0.01 & 0.01 & 0.02 & 0.01 & 0.03 & 0.01 & 0.02 & 0.02 & 0.01 & 0.02 \\
\hline$M n$ & 0.00 & 0.00 & 0.00 & 0.00 & 0.00 & 0.00 & 0.00 & 0.00 & 0.00 & 0.00 & 0.00 \\
\hline $\mathrm{Mg}$ & 0.01 & 0.00 & 0.00 & 0.00 & 0.00 & 0.00 & 0.00 & 0.00 & 0.00 & 0.00 & 0.00 \\
\hline $\mathrm{Ca}$ & 0.16 & 0.30 & 0.24 & 0.55 & 0.17 & 0.00 & 0.02 & 0.66 & 0.27 & 0.05 & 0.70 \\
\hline $\mathrm{Na}$ & 0.75 & 0.66 & 0.71 & 0.43 & 0.74 & 0.57 & 0.49 & 0.31 & 0.64 & 0.57 & 0.29 \\
\hline$x$ & 0.08 & 0.04 & 0.04 & 0.01 & 0.06 & 0.41 & 0.47 & 0.02 & 0.07 & 0.40 & 0.01 \\
\hline $\mathrm{Cr}$ & 0.00 & 0.00 & 0.00 & 0.00 & 0.00 & 0.00 & 0.00 & 0.00 & 0.00 & 0.00 & 0.00 \\
\hline $\mathrm{Ni}$ & 0.00 & 0.00 & 0.00 & 0.00 & 0.00 & 0.00 & 0.00 & 0.00 & 0.01 & 0.01 & 0.00 \\
\hline 0 & 8.00 & 8.00 & 8.00 & 8.00 & 8.00 & 8.00 & 8.00 & 8.00 & 8.00 & 8.00 & 8.00 \\
\hline Total & 4.99 & 5.00 & 4.99 & 4.99 & 4.98 & 5.00 & 4.98 & 4.99 & 4.99 & 5.02 & 5.00 \\
\hline $\mathrm{Ca} / \mathrm{Ca}+\mathrm{Na}$ & 0.18 & 0.31 & 0.25 & 0.56 & 0.19 & 0.01 & 0.03 & 0.68 & 0.29 & 0.08 & 0.71 \\
\hline
\end{tabular}

Footnote:

$c=$ Core

$R / C=$ Intermediate core-rim

$R=\operatorname{Rim}$

$G=$ Groundmass 
Table 2 (continued).

\begin{tabular}{|c|c|c|c|c|c|c|c|c|}
\hline $8018-$ & 8018- & 801B- & $801 \mathrm{C}-$ & $801 \mathrm{C}-$ & $801 \mathrm{C}-$ & $801 \mathrm{C}-$ & $801 \mathrm{C}-$ & $801 C$ - \\
\hline 44R-3. & $44 R-3$ & $44 R-3$ & $1 R-1$ & $1 R=1$ & $1 R-1$ & $2 R-5$ & $2 R-5$ & $2 R-5$ \\
\hline $28-33$ & $28-33$ & $28-33$ & $14-20$ & $14-20$ & $14-20$ & $101-106$ & $101-106$ & $101-106$ \\
\hline$R$ & G & c & $R$ & 6 & $G$ & $R$ & c & G \\
\hline 54.33 & 65.61 & 52.04 & 65.50 & 65.44 & 63.31 & 53.01 & 53.01 & 66.21 \\
\hline 0.16 & 0.03 & 0.19 & 0.18 & 0.02 & 0.05 & 0.13 & 0.13 & 0.08 \\
\hline 27.78 & 19.64 & 29.73 & 19.87 & 18.59 & 21.96 & 29.55 & 29.55 & 18.84 \\
\hline 0.81 & 0.33 & 0.35 & 0.30 & 0.98 & 0.37 & 0.69 & 0.69 & 0.29 \\
\hline 0.02 & 0.01 & 0.04 & 0.05 & 0.00 & 0.00 & 0.08 & 0.08 & 0.00 \\
\hline 0.04 & 0.00 & 0.01 & 0.00 & 0.43 & 0.00 & 0.00 & 0.00 & 0.00 \\
\hline 11.57 & 1.12 & 13.06 & 1.75 & 0.03 & 3.98 & 13.00 & 13.00 & 0.39 \\
\hline 4.58 & 7.11 & 3.96 & 7.92 & 3.39 & 8.12 & 3.79 & 3.79 & 5.99 \\
\hline 0.58 & 5.81 & 0.31 & 4.18 & 11.46 & 1.90 & 0.33 & 0.33 & 7.84 \\
\hline 0.00 & 0.02 & 0.00 & 0.00 & 0.00 & 0.04 & 0.14 & 0.14 & 0.09 \\
\hline 0.26 & 0.00 & 0.00 & 0.08 & 0.18 & 0.00 & 0.06 & 0.06 & 0.02 \\
\hline 0.00 & 0.00 & 0.00 & 0.00 & 0.00 & 0.00 & 0.00 & 0.00 & 0.00 \\
\hline 100.12 & 99.69 & 99.68 & 99.84 & 100.53 & 99.73 & 100.79 & 100.79 & 99.73 \\
\hline 2.47 & 2.95 & 2.38 & 2.93 & 2.95 & 2.83 & 2.39 & 2.39 & 2.99 \\
\hline 0.01 & 0.00 & 0.01 & 0.01 & 0.00 & 0.00 & 0.00 & 0.00 & 0.00 \\
\hline 1.49 & 1.04 & 1.60 & 1.05 & 1.00 & 1.16 & 1.57 & 1.57 & 1.00 \\
\hline 0.03 & 0.01 & 0.01 & 0.01 & 0.04 & 0.01 & 0.03 & 0.03 & 0.01 \\
\hline 0.00 & 0.00 & 0.00 & 0.00 & 0.00 & 0.00 & 0.00 & 0.00 & 0.00 \\
\hline 0.00 & 0.00 & 0.00 & 0.00 & 0.03 & 0.00 & 0.00 & 0.00 & 0.00 \\
\hline 0.56 & 0.05 & 0.64 & 0.08 & 0.00 & 0.19 & 0.63 & 0.63 & 0.02 \\
\hline 0.40 & 0.62 & 0.35 & 0.69 & 0.30 & 0.70 & 0.33 & 0.33 & 0.52 \\
\hline 0.03 & 0.33 & 0.02 & 0.24 & 0.66 & 0.11 & 0.02 & 0.02 & 0.45 \\
\hline 0.00 & 0.00 & 0.00 & 0.00 & 0.00 & 0.00 & 0.01 & 0.01 & 0.00 \\
\hline 0.01 & 0.00 & 0.00 & 0.00 & 0.01 & 0.00 & 0.00 & 0.00 & 0.00 \\
\hline 8.00 & 8.00 & 8.00 & 8.00 & 8.00 & 8.00 & 8.00 & 8.00 & 8.00 \\
\hline 5.00 & 5.01 & 5.00 & 5.01 & 4.98 & 5.00 & 4.99 & 4.99 & 5.00 \\
\hline 0.58 & 0.08 & 0.65 & 0.11 & 0.00 & 0.21 & 0.65 & 0.65 & 0.03 \\
\hline
\end{tabular}

roxenes cannot be the same as that outlined earlier in this section as there is insufficient $\mathrm{Al}$ in octahedral coordination. Flower (1974) determined the phase relations of titan-acmite and suggested a substitution of the type $\mathrm{TiY}^{4+} \mathrm{MZ}^{3+}=\mathrm{MY}^{3+} \mathrm{SiZ}^{4+}$, where $\mathrm{Y}=$ octahedral co-ordination, $\mathrm{Z}=$ tetrahedral co-ordination and $\mathrm{M}=$ cation. This substitution implies replacement of $\mathrm{Si}^{4+}$ by a trivalent cation, but in the Leg 129 alkali pyroxenes there is limited substitution in these sites and therefore this cannot be an accepted substitution in this particular case. Deer et al. (1978) showed that high $\mathrm{Ti}^{4+}$ contents in alkali pyroxenes are usually accompanied by lower $\mathrm{Fe}^{3+}$ and higher $\mathrm{Mg}^{2+}+\mathrm{Fe}^{2+}$. Leg 129 alkali pyroxenes, however, have insufficient $\mathrm{Fe}^{3+}$ to charge-balance the entry of $\mathrm{Na}^{+}$into the structure and there is probably a substitution of the type (Ferguson, 1977) $\mathrm{NaFe}^{3+} \mathrm{Si}_{2} \mathrm{O}_{6}$ (aegirine) $=\mathrm{Na}\left(\mathrm{MgFe}^{2+}\right)_{0.5} \mathrm{Ti}_{0.5} \mathrm{O}_{6}$ (neptunite) .

The occurrence of strongly alkalic rims to both the pyroxenes and the feldspars suggests that rim growth was probably broadly synchronous and occurred during the late-stage hydrous crystallization of the alkalic rocks along with amphibole and biotite. 
G. ROWBOTHAM, P. A. FLOYD

Table 3. Clinopyroxene analyses from alkalic dolerites and basalts, Holes $800 \mathrm{~A}, 801 \mathrm{~B}$, and $801 \mathrm{C}$.

\begin{tabular}{|c|c|c|c|c|c|c|c|c|c|c|c|}
\hline Hole & $800 \mathrm{~A}-$ & $800 \mathrm{~A}$ - & $800 \mathrm{~A}-$ & $800 \mathrm{~A}$ - & $800 \mathrm{~A}-$ & $801 \mathrm{~B}$ & $801 \mathrm{~B}$ & $801 \mathrm{~B}$ & $801 \mathrm{~B}$ & $801 \mathrm{~B}$ & $801 \mathrm{~B}$ \\
\hline Core/section & $58 R-1$ & $58 R-1$ & $58 R-1$ & $58 R-2$ & $58 R-2$ & $41 R-1$ & $41 R-1$ & $41 R-1$ & $43 R-1$ & 43R-1. & $43 R-1$ \\
\hline Interval $(\mathrm{cm})$ & $86-91$ & $86-91$ & $86-91$ & $67-73$ & $67-73$ & $130-136$ & $130-136$ & $130-136$ & $4-9$ & $4-9$ & $4-9$ \\
\hline & c & $R / C$ & $R$ & c & $\mathbf{R}$ & c & c & $\mathbf{R}$ & R & c & $\mathbf{R}$ \\
\hline $\mathrm{SiO}_{2}$ & 49.12 & 50.50 & 50.85 & 49.23 & 51.13 & 48.60 & 45.10 & 48.81 & 49.01 & 44.28 & 49.45 \\
\hline $\mathrm{TiO}_{2}$ & 1.98 & 1.57 & 0.99 & 1.73 & 1.20 & 2.13 & 3.73 & 1.92 & 1.72 & 4.96 & 1.74 \\
\hline $\mathrm{Al}_{2} \mathrm{O}_{3}$ & 4.43 & 3.28 & 1.70 & 3.68 & 1.93 & 4.50 & 6.31 & 4.01 & 4.04 & 7.89 & 3.98 \\
\hline $\mathrm{FeO}$ & 8.67 & 9.69 & 10.79 & 9.45 & 8.60 & 9.27 & 8.80 & 10.33 & 9.80 & 8.79 & 9.47 \\
\hline Mno & 0.14 & 0.31 & 0.39 & 0.28 & 0.18 & 0.14 & 0.14 & 0.26 & 0.25 & 0.11 & 0.31 \\
\hline $\mathrm{MgO}$ & 14.03 & 13.80 & 13.80 & 13.04 & 14.65 & 12.14 & 11.15 & 11.60 & 12.22 & 10.82 & 12.70 \\
\hline $\mathrm{CaO}$ & 20.29 & 19.70 & 19.34 & 21.34 & 21.07 & 21.90 & 22.37 & 21.34 & 21.61 & 22.24 & 21.80 \\
\hline $\mathrm{Na}_{2} \mathrm{O}$ & 0.51 & 0.44 & 0.56 & 0.51 & 0.50 & 0.69 & 0.56 & 0.74 & 0.67 & 0.82 & 0.52 \\
\hline $\mathrm{K}_{2} \mathrm{O}$ & 0.06 & 0.04 & 0.10 & 0.06 & 0.00 & 0.00 & 0.06 & 0.07 & 0.00 & 0.05 & 0.00 \\
\hline $\mathrm{Cr}_{2} \mathrm{O}_{3}$ & 0.30 & 0.03 & 0.00 & 0.04 & 0.03 & 0.04 & 0.10 & 0.15 & 0.00 & 0.10 & 0.08 \\
\hline NiO & 0.00 & 0.10 & 0.00 & 0.10 & 0.19 & 0.40 & 0.00 & 0.05 & 0.24 & 0.09 & 0.45 \\
\hline 0 & 0.00 & 0.00 & 0.00 & 0.00 & 0.00 & 0.00 & 0.00 & 0.00 & 0.00 & 0.00 & 0.00 \\
\hline TOTAL $\%$ & 99.56 & 99.72 & 98.43 & 99.45 & 99.46 & 99.81 & 98.31 & 99.29 & 99.57 & 100.15 & 100.49 \\
\hline Recalculation & to $6(0)$ & & & & & & & & & & \\
\hline si & 1.84 & 1.89 & 1.94 & 1.86 & 1.92 & 1.84 & 1.74 & 1.86 & 1.86 & 1.68 & 1.86 \\
\hline $\mathrm{Ti}$ & 0.06 & 0.04 & 0.03 & 0.05 & 0.03 & 0.06 & 0.11 & 0.06 & 0.05 & 0.14 & 0.05 \\
\hline Al & 0.20 & 0.15 & 0.08 & 0.16 & 0.09 & 0.20 & 0.29 & 0.18 & 0.18 & 0.35 & 0.18 \\
\hline $\mathrm{Fe}$ & 0.27 & 0.30 & 0.33 & 0.30 & 0.27 & 0.29 & 0.28 & 0.33 & 0.31 & 0.28 & 0.30 \\
\hline Mn & 0.00 & 0.01 & 0.01 & 0.01 & 0.01 & 0.00 & 0.01 & 0.01 & 0.01 & 0.00 & 0.01 \\
\hline $\mathrm{Mg}$ & 0.79 & 0.77 & 0.78 & 0.74 & 0.82 & 0.68 & 0.64 & 0.66 & 0.69 & 0.61 & 0.71 \\
\hline $\mathrm{Ca}$ & 0.82 & 0.80 & 0.79 & 0.87 & 0.85 & 0.89 & 0.92 & 0.87 & 0.88 & 0.90 & 0.88 \\
\hline $\mathrm{Na}$ & 0.04 & 0.03 & 0.04 & 0.04 & 0.04 & 0.05 & 0.04 & 0.06 & 0.05 & 0.06 & 0.04 \\
\hline K & 0.00 & 0.00 & 0.00 & 0.00 & 0.00 & 0.00 & 0.00 & 0.00 & 0.00 & 0.00 & 0.00 \\
\hline $\mathrm{Cr}$ & 0.00 & 0.00 & 0.00 & 0.00 & 0.00 & 0.00 & 0.00 & 0.01 & 0.00 & 0.00 & 0.00 \\
\hline $\mathrm{Ni}$ & 0.01 & 0.00 & 0.00 & 0.00 & 0.01 & 0.01 & 0.00 & 0.00 & 0.01 & 0.00 & 0.01 \\
\hline 0 & 6.00 & 6.00 & 6.00 & 6.00 & 6.00 & 6.00 & 6.00 & 6.00 & 6.00 & 6.00 & 6.00 \\
\hline Total & 4.02 & 4.01 & 4.00 & 4.03 & 4.02 & 4.03 & 4.03 & 4.03 & 4.03 & 4.04 & 4.03 \\
\hline
\end{tabular}

Footnote:

$c=$ Core

$R / C=$ Intermediate core-rim

$R=\operatorname{Rim}$ 
Table 3 (continued).

\begin{tabular}{ccccccccc}
\hline $805 B$ & $801 B$ & $801 B$ & $801 C$ & $801 C$ & $801 C$ & $801 C$ & $801 C$ & $801 C$ \\
$44 R-2$, & $44 R-2$, & \multicolumn{1}{c}{$44 R-2}$, & $1 R-1$, & $1 R-1$, & $1 R-1$, & $2 R-5$, & $2 R-5$, & $2 R-5$. \\
$140-145$ & $140-145$ & $140-145$ & $14-20$ & $14-20$ & $14-20$ & $101-106$ & $101-106$ & $101-106$ \\
\hline$R$ & $R / C$ & $C$ & $C$ & $R$ & $C$ & $C$ & $R$ & $C$ \\
44.54 & 45.59 & 49.71 & 42.23 & 50.59 & 51.31 & 45.17 & 44.89 & 44.43 \\
4.46 & 3.60 & 1.52 & 5.91 & 1.43 & 1.30 & 4.00 & 3.97 & 4.06 \\
7.43 & 6.76 & 3.06 & 9.26 & 3.25 & 2.60 & 7.64 & 7.28 & 7.20 \\
9.27 & 9.49 & 11.72 & 8.94 & 8.57 & 8.94 & 9.55 & 9.53 & 9.69 \\
0.10 & 0.18 & 0.17 & 0.22 & 0.26 & 0.34 & 0.16 & 0.06 & 0.04 \\
10.63 & 10.65 & 11.25 & 10.61 & 13.55 & 12.91 & 10.67 & 11.00 & 10.66 \\
22.24 & 22.07 & 21.31 & 22.16 & 21.82 & 22.07 & 22.67 & 21.44 & 22.27 \\
0.78 & 0.69 & 0.72 & 0.85 & 0.68 & 0.78 & 0.57 & 0.64 & 0.72 \\
0.00 & 0.00 & 0.06 & 0.00 & 0.00 & 0.03 & 0.03 & 0.10 & 0.02 \\
0.11 & 0.07 & 0.00 & 0.02 & 0.00 & 0.17 & 0.10 & 0.13 & 0.06 \\
1.02 & 0.28 & 0.00 & 0.00 & 0.00 & 0.00 & 0.00 & 0.00 & 0.61 \\
0.00 & 0.00 & 0.00 & 0.00 & 0.00 & 0.00 & 0.00 & 0.00 & 0.00 \\
100.58 & 99.38 & 99.51 & 100.20 & 100.13 & 100.46 & 100.55 & 99.59 & 99.76
\end{tabular}

\begin{tabular}{lllllllll}
1.69 & 1.74 & 1.90 & 1.61 & 1.89 & 1.91 & 1.71 & 1.71 & 1.70 \\
0.13 & 0.10 & 0.04 & 0.17 & 0.04 & 0.04 & 0.11 & 0.11 & 0.12 \\
0.33 & 0.30 & 0.14 & 0.42 & 0.14 & 0.11 & 0.34 & 0.35 & 0.33 \\
0.29 & 0.03 & 0.37 & 0.28 & 0.27 & 0.28 & 0.30 & 0.30 & 0.31 \\
0.00 & 0.01 & 0.01 & 0.01 & 0.01 & 0.01 & 0.01 & 0.00 & 0.00 \\
0.60 & 0.61 & 0.64 & 0.60 & 0.75 & 0.72 & 0.60 & 0.62 & 0.61 \\
0.90 & 0.90 & 0.87 & 0.90 & 0.87 & 0.88 & 0.92 & 0.87 & 0.91 \\
0.06 & 0.05 & 0.05 & 0.06 & 0.05 & 0.06 & 0.04 & 0.05 & 0.05 \\
0.00 & 0.00 & 0.00 & 0.00 & 0.00 & 0.00 & 0.00 & 0.01 & 0.00 \\
0.00 & 0.00 & 0.00 & 0.00 & 0.00 & 0.01 & 0.00 & 0.00 & 0.00 \\
0.03 & 0.01 & 0.00 & 0.00 & 0.00 & 0.00 & 0.00 & 0.00 & 0.02 \\
6.00 & 6.00 & 6.00 & 6.00 & 6.00 & 6.00 & 6.00 & 6.00 & 6.00 \\
4.04 & 3.76 & 4.02 & 4.05 & 4.02 & 4.02 & 4.03 & 4.03 & 4.05 \\
\hline
\end{tabular}

\section{Amphiboles}

Brown magmatic amphiboles (Table 5) occur in minor proportions in both Hole 800A dolerites and the coarser interiors of the olivinerich microdolerites of Site 801 . Only samples from Hole 801 have been analyzed and are not characteristic of common magmatic calciferous amphiboles (Fig. 6). They are aluminum-poor with a maximum cation content of 0.34 cations $/ 23$ oxygens in the formula unit, all of which is allocated to the tetrahedral sites in the cfu recalculation (Table 5). Calculation of the ferric iron content (method of Droop, 1987) yielded a range of $\mathrm{Fe}^{3+}$ values between 0.65 and $1.28 \mathrm{cfu}$. Both of these features are unusual in igneous clinoamphiboles (Hawthorne, 1981), and following the nomenclature of Leake (1978) these minerals are winchite-ferriwinchite in composition $\left(\mathrm{CaNa}\left(\mathrm{MgFe}^{2+}\right)_{4} \mathrm{Fe}^{3+}\right.$ 
Table 4. Alkali pyroxene analyses from alkalic basalts, Holes 801B and 801C.

\begin{tabular}{|c|c|c|c|c|c|c|c|c|c|c|c|}
\hline Hole & 801B- & 8018- & 801B- & $801 c$ - & 801C. & 801C- & 801C. & 801C. & 801C. & 801C- & $801 \mathrm{C}-$ \\
\hline Core/section & $43 R-1$ & $43 R-1$ & $43 R-1$ & 1R-1. & $1 \mathrm{R}-1$ & 1R-1 & 1R-1. & $1 R-1$ & $1 R-1$ & $1 R-1$ & $1 R-1$ \\
\hline Interval $(\mathrm{cm})$ & 4.9 & 4.9 & 4.9 & $14-20$ & $14-20$ & $14-20$ & $14-20$ & 14.20 & $14-20$ & $14-20$ & $14-20$ \\
\hline & R & R & $\mathrm{R}$ & R & R & $E / R$ & R & R & $E / R$ & R & R \\
\hline $\mathrm{SiO}_{2}$ & 52.84 & 53.07 & 52.83 & 50.27 & 50.04 & 50.71 & 50.51 & 50.08 & 50.96 & 51.10 & 50.88 \\
\hline $\mathrm{TiO}_{2}$ & 3.83 & 3.81 & 3.56 & 3.74 & 2.71 & 4.79 & 4.74 & 4.52 & 4.90 & 5.10 & 5.11 \\
\hline${ }^{A L} 2_{2} \mathrm{O}_{3}$ & 0.46 & 0.78 & 0.72 & 0.22 & 0.00 & 0.20 & 0.47 & 0.47 & 0.31 & 0.47 & 0.21 \\
\hline $\mathrm{Fe}_{2} \mathrm{O}_{3}$ & 23.15 & 24.57 & 22.70 & 14.03 & 8.50 & 12.08 & 11.38 & 11.61 & 13.98 & 11.60 & 11.76 \\
\hline $\mathrm{FeO}$ & 4.06 & 2.79 & 4.74 & 11.88 & 17.29 & 13.36 & 13.65 & 13.11 & 11.90 & 14.00 & 13.54 \\
\hline Mno & 0.33 & 0.20 & 0.23 & 0.40 & 0.98 & 0.63 & 0.66 & 0.32 & 0.30 & 0.59 & 0.40 \\
\hline $\mathrm{MgO}$ & 0.81 & 0.76 & 0.66 & 0.11 & 0.14 & 0.09 & 0.16 & 0.15 & 0.10 & 0.20 & 0.10 \\
\hline $\mathrm{CaO}$ & 2.98 & 2.67 & 2.60 & 10.22 & 14.45 & 9.44 & 9.21 & 8.82 & 7.85 & 9.85 & 10.08 \\
\hline $\mathrm{Na}_{2} \mathrm{O}$ & 12.22 & 12.75 & 12.27 & 8.12 & 5.44 & 8.33 & 8.25 & 8.40 & 9.24 & 8.22 & 8.28 \\
\hline$k_{2} \mathrm{O}$ & 0.10 & 0.00 & 0.00 & 0.08 & 0.00 & 0.02 & 0.00 & 0.00 & 0.02 & 0.00 & 0.00 \\
\hline TOTAL\% & 100.78 & 101.39 & 100.30 & 99.08 & 99.55 & 99.65 & 99.03 & 97.47 & 99.55 & 100.69 & 100.36 \\
\hline \multicolumn{12}{|c|}{ Recalculation to $6(0)$} \\
\hline si & 2.00 & 1.99 & 2.01 & 1.99 & 2.00 & 1.99 & 2.00 & 2.00 & 1.99 & 1.99 & 1.99 \\
\hline $\mathrm{Ti}$ & 0.11 & 0.11 & 0.03 & 0.11 & 0.08 & 0.14 & 0.14 & 0.14 & 0.14 & 0.15 & 0.15 \\
\hline AL & 0.02 & 0.04 & 0.10 & 0.01 & 0.00 & 0.01 & 0.02 & 0.02 & 0.01 & 0.02 & 0.01 \\
\hline $\mathrm{Fe}^{3+}$ & 0.66 & 0.69 & 0.65 & 0.42 & 0.26 & 0.36 & 0.34 & 0.35 & 0.41 & 0.33 & 0.35 \\
\hline $\mathrm{Fe}^{2+}$ & 0.13 & 0.09 & 0.15 & 0.39 & 0.58 & 0.44 & 0.45 & 0.44 & 0.39 & 0.46 & 0.44 \\
\hline Mn & 0.01 & 0.01 & 0.01 & 0.01 & 0.03 & 0.02 & 0.02 & 0.01 & 0.01 & 0.02 & 0.01 \\
\hline $\mathrm{Mg}$ & 0.05 & 0.04 & 0.04 & 0.01 & 0.01 & 0.01 & 0.01 & 0.01 & 0.01 & 0.01 & 0.01 \\
\hline ca & 0.12 & 0.11 & 0.11 & 0.43 & 0.62 & 0.40 & 0.39 & 0.38 & 0.33 & 0.41 & 0.42 \\
\hline $\mathrm{Na}$ & 0.90 & 0.93 & 0.91 & 0.62 & 0.42 & 0.64 & 0.63 & 0.65 & 0.70 & 0.62 & 0.63 \\
\hline $\mathrm{k}$ & 0.01 & 0.00 & 0.00 & 0.00 & 0.00 & 0.00 & 0.00 & 0.00 & 0.00 & 0.00 & 0.00 \\
\hline 0 & 6.00 & 6.00 & 6.00 & 6.00 & 6.00 & 6.00 & 6.00 & 6.00 & 6.00 & 6.00 & 6.00 \\
\hline Total & 4.00 & 4.00 & 4.00 & 4.00 & 4.00 & 4.00 & 4.00 & 4.00 & 4.00 & 4.00 & 4.00 \\
\hline
\end{tabular}

Footnote:

$R=\operatorname{Rim}$

$E / R=$ Extreme $r i m$

$\mathrm{Si}_{8} \mathrm{O}_{22}(\mathrm{OH})_{2}$ end-member composition) with limited solid-solution toward katophorite $\left(\mathrm{NaCaNa}\left[\mathrm{MgFe}^{2+}\right]_{4} \mathrm{Fe}^{3+} \mathrm{Si}_{7} \mathrm{AlO}_{22}[\mathrm{OH}]_{2}\right)$. These end members imply the two coupled substitutions:

$\begin{array}{ll}\mathrm{Ca}\left(\mathrm{MgFe}^{2+}\right) & = \\ \text { tremolite-actinolite } & \begin{array}{l}\mathrm{Na} \mathrm{Fe} \\ \text { winchite-ferriwinchite }\end{array}\end{array}$

and
$($ vacancy $\mathrm{A}$ site $)+\mathrm{Si}=\mathrm{NaA}+\mathrm{Al}_{2}$. winchite

katophorite
In addition to these dominant substitutions, $\mathrm{Fe}-\mathrm{Mg}$ replacement is limited $\left(\mathrm{Mg} / \mathrm{Mg}+\mathrm{Fe}^{2+}\right.$ ratio $\left.=0.44-0.64\right)$. The rims of some of the crystals have higher $\mathrm{Fe}^{3+} / \mathrm{Fe}^{2+}$ ratios, which may reflect increasing $\mathrm{fO}_{2}$ as hydrous crystallization proceeded. Other chemical factors exhibited by the amphiboles, such as low Ti concentrations $(0.09-$ $0.17 \mathrm{cfu}$ ) which incompletely filled the A sites in the lattice and limited replacement of $\mathrm{Si}$ by $\mathrm{Al}$ in the tetrahedral sites, are generally thought to reflect crystallization at relatively low magmatic temperatures (Helz, 1973; 1980). Petrographically they are late in development and herald a change to more hydrous and oxidizing conditions of crystallization. 
Table 4 (continued).

\begin{tabular}{|c|c|c|c|c|c|c|}
\hline $801 C-$ & $801 C$ - & $801 \mathrm{C}-$ & 801C- & $801 \mathrm{C}-$ & 801B- & 801B- \\
\hline $1 R-1$ & R-1. & R-1 & RR-1. & $1 R-1$ & 44R-2. & $44 R-2$ \\
\hline $14-20$ & $14-20$ & $14-20$ & $14-20$ & $14-20$ & $140-145$ & $140-145$ \\
\hline$E / R$ & R & R & $E / R$ & $E / R$ & $\mathrm{R}$ & $E / R$ \\
\hline 50.57 & 50.44 & 50.44 & 51.10 & 50.97 & 50.74 & 50.88 \\
\hline 4.46 & 4.28 & 4.28 & 4.47 & 4.87 & 5.11 & 3.97 \\
\hline 0.45 & 0.64 & 0.64 & 0.64 & 0.69 & 2.06 & 2.39 \\
\hline 19.27 & 10.99 & 10.99 & 14.14 & 13.47 & 20.67 & 22.65 \\
\hline 6.75 & 14.24 & 14.24 & 11.70 & 11.83 & 4.74 & 3.32 \\
\hline 0.29 & 0.81 & 0.81 & 0.43 & 0.52 & 0.30 & 0.25 \\
\hline 0.23 & 0.35 & 0.35 & 0.19 & 0.17 & 1.80 & 2.20 \\
\hline 6.51 & 11.16 & 11.16 & 8.52 & 8.33 & 2.01 & 1.90 \\
\hline 10.48 & 7.37 & 7.37 & 9.00 & 9.05 & 11.72 & 11.75 \\
\hline 0.03 & 0.00 & 0.00 & 0.00 & 0.01 & 0.03 & 0.00 \\
\hline 99.04 & 100.28 & 100.28 & 100.19 & 99.91 & 99.18 & 99.31 \\
\hline 1.97 & 1.98 & 1.98 & 1.99 & 1.99 & 1.94 & 1.94 \\
\hline 0.13 & 0.13 & 0.13 & 0.13 & 0.14 & 0.15 & 0.11 \\
\hline 0.02 & 0.03 & 0.03 & 0.03 & 0.03 & 0.09 & 0.11 \\
\hline 0.57 & 0.32 & 0.32 & 0.41 & 0.40 & 0.60 & 0.65 \\
\hline 0.22 & 0.47 & 0.47 & 0.38 & 0.39 & 0.15 & 0.11 \\
\hline 0.01 & 0.03 & 0.03 & 0.01 & 0.02 & 0.01 & 0.01 \\
\hline 0.01 & 0.02 & 0.02 & 0.01 & 0.01 & 0.10 & 0.13 \\
\hline 0.27 & 0.47 & 0.47 & 0.36 & 0.35 & 0.08 & 0.13 \\
\hline 0.79 & 0.56 & 0.56 & 0.68 & 0.68 & 0.87 & 0.08 \\
\hline 0.00 & 0.00 & 0.00 & 0.00 & 0.00 & 0.00 & 0.87 \\
\hline 6.00 & 6.00 & 6.00 & 6.00 & 6.00 & 6.00 & 6.00 \\
\hline 4.00 & 4.00 & 4.00 & 4.00 & 4.00 & 4.00 & 4.00 \\
\hline
\end{tabular}

\section{Biotites}

In the Hole $800 \mathrm{~A}$ dolerites, biotite (Table 6 ) developed both as a late-stage magmatic phase, replacing brown amphibole, as well as post-magmatically in response to contact metamorphism, nucleating on titanomagnetite and replacing smectite-altered interstitial areas. Site 801 basalts contain magmatic biotite more closely associated with primary minerals, occurring as discrete euhedral crystals, as well as nucleating on opaque phases.

Chemically, the very late-stage biotites (Hole 800A) are characterized by relatively low $\mathrm{Ti}^{4+}(0.49-0.68 \mathrm{cfu})$ and $\mathrm{Al}^{3+}$ contents $(1.43-$ $2.19 \mathrm{cfu})$, deficiencies in the tetrahedral sites $(\mathrm{Si}+\mathrm{Al}<8.00 \mathrm{cfu})$ and to a lesser extent in the interlayer sites, and restricted $\mathrm{Mg} / \mathrm{Mg}+\mathrm{Fe}$ ratios $(0.53-0.70)$ (Fig. 7). On the other hand, the magmatic biotites from Holes $801 \mathrm{~B}$ and $801 \mathrm{C}$ have greater Ti contents (up to $1.0 \mathrm{cfu}$ ) in the octahedral sites, and have tetrahedral sites completely occupied by $\mathrm{Si}^{4+}$ and $\mathrm{Al}^{3+}$, and a wider range of $\mathrm{Mg} / \mathrm{Mg}+\mathrm{Fe}$ values. In all the Site 801 samples there is minor replacement of $\mathrm{K}^{+}$by $\mathrm{Na}^{+}$in the interlayer site (up to $0.36 \mathrm{cfu}$ ) which is not seen to the same extent in the Hole $800 \mathrm{~A}$ samples. In addition, there seems to be an inverse relationship between $\mathrm{Ti}^{4+}$ and total $\mathrm{Fe}^{*}$ in the Site 801 biotites. Roberts (1976) suggested that increased entry of $\mathrm{Ti}^{4+}$ into the biotite structure was favored by higher temperatures, and this interpretation is supported by the observations in the present study. Biotites from Site 801 have the highest $\mathrm{Ti}^{4+}$ contents and appear to be magmatic in development, whereas the Hole $800 \mathrm{~A}$ biotites were probably developed at lower temperatures post-magmatically and/or very late in the crystallization sequence.

\section{Tholeiitic Basalts}

\section{Plagioclases}

Relative to the feldspars from the alkalic rocks described previously, the plagioclases from the tholeiites of Holes $801 \mathrm{C}$ and $802 \mathrm{~A}$ do not exhibit the same compositional range and lack the development of alkali feldspar rims (Table 7). In broad terms they are mainly bytownite-calcic labradorite in composition $\left(\mathrm{An}_{90} \mathrm{An}_{57}\right)$, although the rims of individual crystals may be quite sodic $\left(\mathrm{An}_{24}\right)$.

Plagioclases in the geochemically primitive, plagioclase-olivinespinel phyric tholeiites of Hole $801 \mathrm{C}\left(\mathrm{Mg}^{*}=72-60\right.$; Floyd and Castillo, this volume) characteristically occur in three generations: glomerophyric clusters, microphenocrysts, and quenched variolitic intergrowths with clinopyroxene (for example, Sample 801C-6R-4, 49-54 cm, Table 6). The glomerocrysts are the most anorthitic $\left(\mathrm{An}_{90} \mathrm{Ab}_{10} \mathrm{Or}_{0}\right)$, and some possess normal zoning out to the rims with $\mathrm{An}_{50}$, although zoning is by no means common in these early crystals. There is broad compositional overlap between the glomerocrysts and the microphenocrysts, although the latter with chrome spinel inclusions have slightly lower An contents $\left(\mathrm{An}_{75-80}\right)$. Variolitic plagioclase is distinctly more sodic and exhibits a greater range $\left(\mathrm{An}_{65-24}\right)$. Groundmass microlites and serrated laths are also systematically more albitic than the microphenocrysts and contain higher contents of $\mathrm{Fe}$ and $\mathrm{Mg}$. There does not seem to be any correlation between An content and minor element abundances as documented for some submarine basalts (Bence et al., 1975).

Both microphenocrystic and groundmass plagioclases within the Hole $802 \mathrm{~A}$ tholeiites show marginally more albitic compositions than those in Hole $801 \mathrm{C}$, although once again, with few exceptions, Or contents are minimal. The most anorthitic compositions are bytownites (up to $\left.A n_{86}\right)$, which can range down to calcic andesine $\left(\mathrm{An}_{50-47}\right)$ in rim zones (Fig. 8). In general, microphenocryst and quench crystals overlap in composition with both commonly exhibiting values $>\mathrm{An}_{70}$. One important petrographic characteristic is the presence in all analyzed plagioclase microphenocrysts of complex zoning patterns (oscillatory and reversed), irrespective of whether they occur in glassy matrix, quench-textured groundmass, or intersertal-textured matrix. For example, a plagioclase microphenocryst analyzed from Sample $802 \mathrm{~A}-59 \mathrm{R}-3,40-44 \mathrm{~cm}$, showed the following variation: from $\mathrm{An}_{84.8} \mathrm{Ab}_{14.5} \mathrm{Or}_{0.0}$ (core) to $\mathrm{An}_{79.5} \mathrm{Ab}_{20.5} \mathrm{Or}_{0.0}$ (intermediate zone) to $\mathrm{An}_{74.9} \mathrm{Ab}_{24.4} \mathrm{Or}_{0.7}$ (rim). The minor change in An content (of about $5 \%$ $\mathrm{An}$ ) between adjacent zones in the crystal is typical of many Hole 802 A plagioclases.

Another feature of plagioclases from the Hole 802A tholeiites is the apparent lack of a correlation between An content and minor components, such as $\mathrm{Fe}$ and $\mathrm{Mg}$ in the feldspar lattice. Many primitive mid-ocean ridge basalts (MORB) containing highly calcic plagioclase may display increasing $\mathrm{Mg}$ abundances with decreasing An content (Bence et al., 1975), whereas Nauru Basin plagioclases from evolved tholeiites show a positive correlation between $\mathrm{Mg}$ and $\mathrm{An}$ (Floyd and Rowbotham, 1986). On the other hand, Kempton et al. (1985) noted a positive correlation of $\mathrm{Mg}$ with An content for phenocrysts, xenocrysts, and groundmass feldspar only when the compositions were below $\mathrm{An}_{79}$ and had a complex relationship in compositions more An-rich than this figure. The compositions of the Hole 802A plagioclases are generally more An-rich than those of the Nauru Basin, between $\mathrm{An}_{75}$ and $\mathrm{An}_{85}$, and fall into the area of more complex relationships as noted by Kempton et al. (1985). 

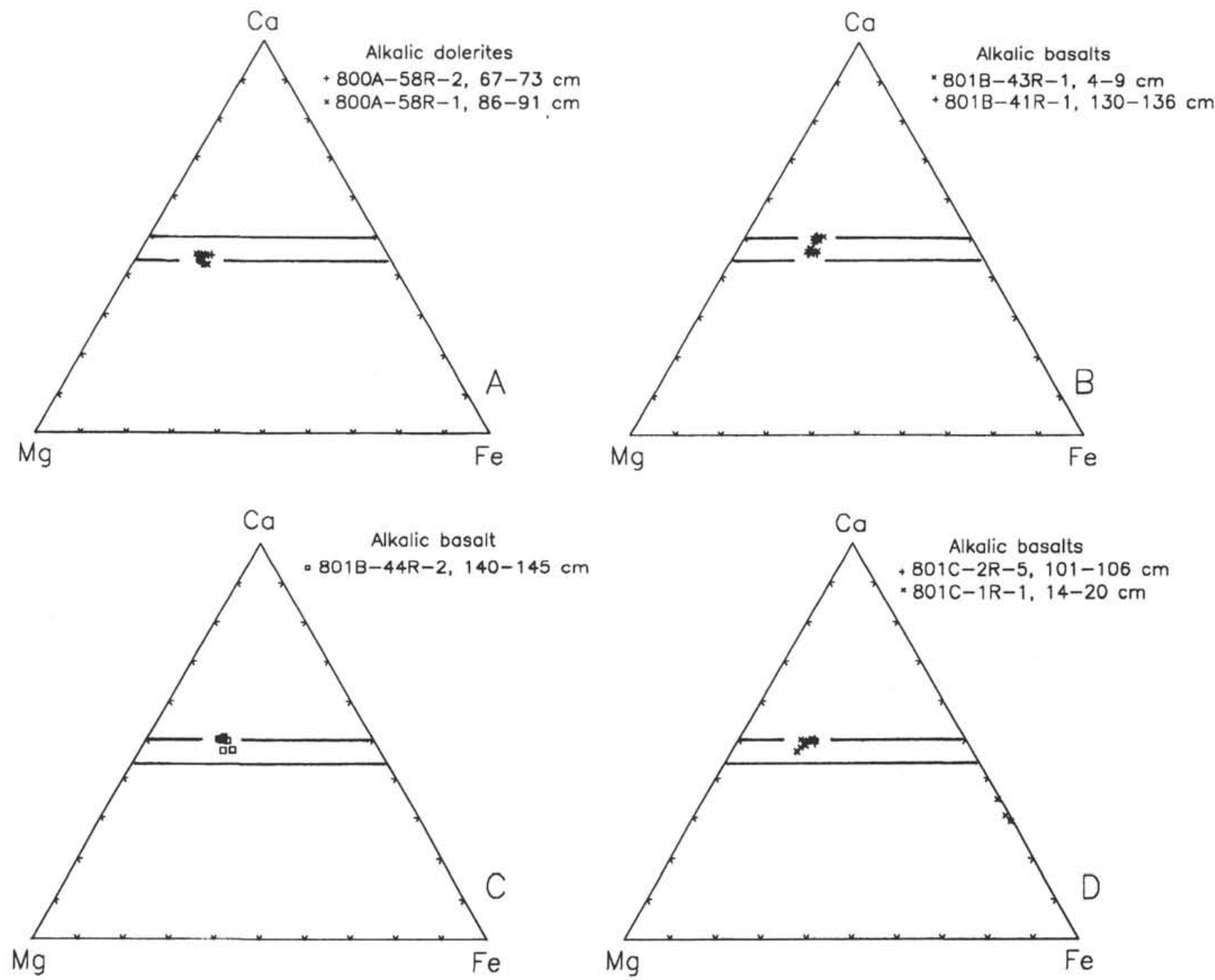

Figure 3. Composition of clinopyroxenes from alkalic dolerites and basalts, in terms of Ca, Fe, and Mg. A. Dolerite sills from Hole 800A. B, C, and D. Various basalt flows from Site 801 .

\section{Pyroxenes}

The pyroxenes in the tholeiitic basalts of Holes $801 \mathrm{C}$ and $802 \mathrm{~A}$ are extremely variable in terms of both quadrilateral and minor element components (Table 8). However, with one exception, core compositions of Hole 802A clinopyroxenes are augite and form a tight cluster around the composition $\mathrm{Ca}_{37.5} \mathrm{Mg}_{51} \mathrm{Fe}_{11.5}$ (Fig. 9). They are very similar to MORB clinopyroxene compositions (for example, Bence et al., 1975), although in this case the limited compositional range reflects the narrow chemical composition exhibited by the bulk rocks, which have essentially uniform $\mathrm{FeO} * / \mathrm{MgO}$ ratios (Floyd et al., this volume). However, composite pyroxene grains from Sample 802A-62R-2, 53$59 \mathrm{~cm}$ do not fit this homogeneous pattern and exhibit wide variations in composition between optically distinguishable segments. The cores of the grains are Ca-poor (for example, $\mathrm{Ca}_{10.0} \mathrm{Mg}_{53.5} \mathrm{Fe}_{36.5}$ ), whereas the rims are more $\mathrm{Ca}$-rich (for example, $\mathrm{Ca}_{30.8} \mathrm{Mg}_{37.7} \mathrm{Fe}_{31.5}$ ). The distribution of the spot analyses (Fig. 9) indicates substitution of the type $\mathrm{Ca}=\mathrm{Mg}, \mathrm{Ca}=\mathrm{Fe}$, and $\mathrm{Mg}=\mathrm{Fe}$, with trends among the quadrilateral components characteristic of compositional variation due to quenching. In terms of the nonquadrilateral components $\mathrm{Ti}, \mathrm{Cr}$, and $\mathrm{Al}$ (Fig. 10), the Hole 802A clinopyroxenes are extremely variable compared to those of the alkalic rocks described previously. Ti abundances are low $(0.01 \mathrm{cfu})$ with $\mathrm{Ti} / \mathrm{Al}$ ratios of approximately $1: 10$, although with the exception of Sample 802A-62R-2, 53-59 cm, within-sample variation is limited. The $\mathrm{Ti}$ and $\mathrm{Cr}$ contents are similar to those of the Nauru Basin clinopyroxenes (Floyd and Rowbotham, 1986) and generally reflect the low $\mathrm{Ti}$ and $\mathrm{Cr}$ contents of the bulk rocks.

The Hole $801 \mathrm{C}$ clinopyroxenes occur as microphenocrysts, sporadically with hour-glass structure, as variolitic fans and, rarely, as clear dendrites within olivine (for example, in Sample 801C-6R-4, 49-54 $\mathrm{cm}$ ). In terms of quadrilateral components (Fig. 9), there is a greater range of variability from sample to sample that reflects wider chemical differences in bulk-rock composition and textural type than in Hole 802A. Microphenocrysts are diopsides and augites, with some crystals showing a marked $\mathrm{Fe}$ enrichment toward grain rims. Sample 801C-7R-2, 122-127 cm exhibits hour-glass structure, with all segments being augite with fairly constant Fe contents. Clinopyroxenes in variolites have been analyzed both along the length and across individual fibers and have a fairly constant composition in terms of quadrilateral components (for example, $\mathrm{Ca}_{44} \mathrm{Mg}_{43} \mathrm{Fe}_{13}$ ). The small groups of granular intersertal clinopyroxenes in flow interiors are commonly zoned with cores of $\mathrm{Ca}_{43} \mathrm{Mg}_{41} \mathrm{Fe}_{16}$ (comparable to the variolitic clinopyroxenes) to extremely $\mathrm{Fe}$-rich rims of $\mathrm{Ca}_{38} \mathrm{Mg}_{12} \mathrm{Fe}_{50}$. The clinopyroxenes of the Hole $801 \mathrm{C}$ tholeiites have marginally greater amounts of the 


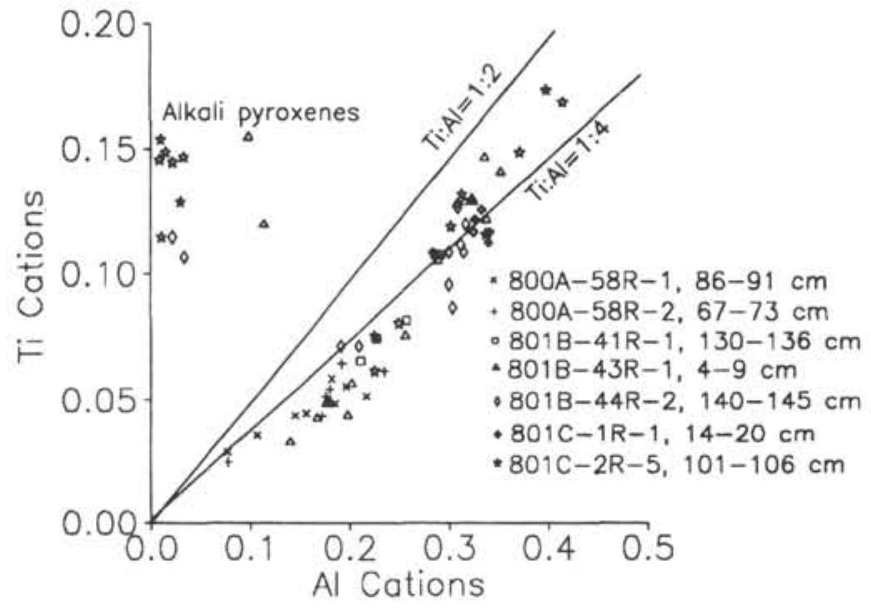

Figure 4. Minor components, Ti and Al, in clinopyroxenes from alkalic dolerite sills and basalt flows, Holes $800 \mathrm{~A}, 801 \mathrm{~B}$, and $801 \mathrm{C}$.

minor, non-quadrilateral components $\mathrm{Ti}$ and $\mathrm{Al}$ compared to Hole $802 \mathrm{~A}$, with a greater spread of Ti/Al ratios (Fig. 10). Clinopyroxenes analyzed in Sample 801C-5R-5, 73-78 cm have the highest Ti content (up to $0.18 \mathrm{cfu}$ ) and $\mathrm{Ti} / \mathrm{Al}$ ratios similar to the alkalic basalts (1:3). The $\mathrm{Cr}$ contents are similar to that found in Hole 802A clinopyroxenes.

Within a number of olivine crystals in the Hole $801 \mathrm{C}$ basalts are rare, highly birefringent, spherical patches composed of small pyroxene dendrites. Probe analyses recalculated to a pyroxene composition give the following average composition: $\left(\mathrm{K}_{0.003} \mathrm{Na}_{0.024} \mathrm{Ca}_{0.919}\right.$ $\left.\mathrm{Mg}_{0.536} \mathrm{Mn}_{0.005} \mathrm{Fe}_{0.182} \mathrm{Ti}_{0.036} \mathrm{Al}_{0.316}\right)_{2.02}\left(\mathrm{Si}_{1.598} \mathrm{Al}_{0.402}\right)_{2} \mathrm{O}_{6}$. The dendrites are highly aluminous with $\mathrm{Al}$ present at high values and commonly in similar proportions in both octahedral and tetrahedral sites suggesting a high proportion of Ca Tschermak's molecule with $\mathrm{R}^{2+}+\mathrm{Si}^{\mathrm{IV}}=\mathrm{Al}^{\mathrm{VI}}$ $+\mathrm{Al}^{\mathrm{IV}}$ substitution. Pyroxenes of this type are of fassaite composition and usually associated with high-pressure conditions (Lovering and White, 1969).

\section{Olivines}

Olivine is commonly replaced completely by alteration products, although rare pristine crystals have been analyzed from two samples (Table 9) in tholeiitic basalts from Hole 801C (cooling unit C20) and Hole $802 \mathrm{~A}$ (cooling unit A5). Sample $801 \mathrm{C}-6 \mathrm{R}-4,49-54 \mathrm{~cm}$ is a primitive group tholeiite with MORB characteristics and contains olivine microphenocrysts that are exceedingly magnesian-rich, reaching compositions of $\mathrm{Fo}_{90}$. Typical across-grain variation is from $\mathrm{Fo}_{80}$ to $\mathrm{Fo}_{88}$ (Fig. 11). Cores are invariably Ni-rich (maximum content 0.72 $\mathrm{wt} \% \mathrm{NiO}$ ), grading to lower values in the rims (minimum content of $0.28 \mathrm{wt} \% \mathrm{NiO}$ ).

Sample 802 A-58R-2, 99-102 cm is an olivine microphyric tholeiite with euhedral olivines embedded in the fresh glass of a pillow rim. There is no detectable chemical zonation in the olivines involving major oxide components, and all have uniform compositions of $\mathrm{Fo}_{79-80}$. The nickel content, however, does vary between 1.0 and 0.2 $\mathrm{wt} \% \mathrm{NiO}$, although there is no systematic variation between core and rim. The olivines were in equilibrium with the original melt, now represented by the enclosing glass. Olivine compositions of $\mathrm{Fo}_{80}$ with iron-magnesium distribution coefficients of 0.30 should be in equilibrium with liquids that have $\mathrm{FeO} / \mathrm{FeO}+\mathrm{MgO}$ ratios of about 0.47 (Roeder, 1974; Longhi et al., 1978), which is close to that calculated (0.48) from the adjacent glass compositions (Table 10).

\section{Spinels}

Spinel occurs as either minute light brown octahedral inclusions within plagioclase or olivine phenocrysts or as larger $(0.5-0.7 \mathrm{~mm})$

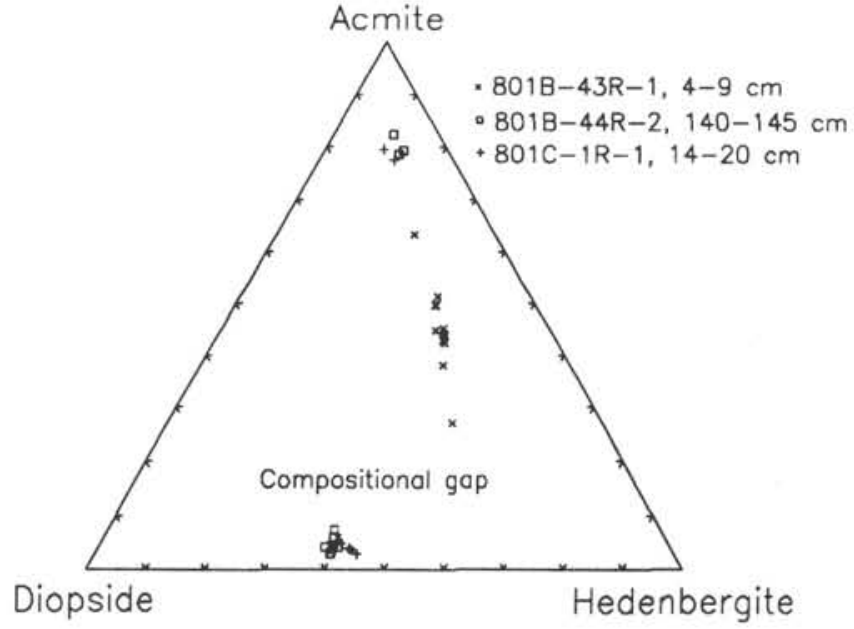

Figure 5. Composition of alkali pyroxenes from alkalic basalts, in terms of acmite, diopside, and hedenbergite components, Holes $801 \mathrm{~B}$ and $801 \mathrm{C}$.

euhedral microphenocrysts associated with olivine in primitive group tholeiites of Hole 801C (for example, Samples 801C-5R-5, 73-78 cm and 801C-6R-4, 49-54) (Table 11). Opaque spinel inclusions also occur within the plagioclase phenocrysts of the alkalic intrusive unit (cooling unit B14) at the base of Hole 801B (Sample 801B-44R-3, 28-33). Spinel paragenesis indicates that they formed at the earliest stages of crystallization.

The opaque spinels within plagioclase crystals from Sample $801 \mathrm{~B}-44 \mathrm{R}-3,28-33 \mathrm{~cm}$ (alkalic basalt) possess highly variable $\mathrm{Al}$ $(2.46-5.05 \mathrm{cfu})$, and $\mathrm{Ti}(1.95-3.56 \mathrm{cfu})$ contents, with smaller variations in total $\mathrm{Fe}(6.11-8.28 \mathrm{cfu})$ and $\mathrm{Cr}(1.67-3.03 \mathrm{cfu}) ; \mathrm{Mg}$ is relatively constant (about $3.20 \mathrm{cfu}$ ). Many of these variations are inter- rather than intracrystalline, making it difficult to assess any systematic zoning within individual crystals. Sigurdsson and Schilling (1976) subdivided spinels from the Mid-Atlantic Ridge into three types: (1) magnesiochromite with $\mathrm{Cr} / \mathrm{Cr}+\mathrm{Al}=0.4-0.5$, (2) titaniferous magnesiochromite, and (3) chrome spinel with $\mathrm{Cr} / \mathrm{Cr}+$ $\mathrm{Al}=0.23-0.27$. On this basis the opaque inclusions in Sample $801 \mathrm{~B}-44 \mathrm{R}-3,28-33 \mathrm{~cm}$ are highly titaniferous spinels and reflect the generally Ti-rich alkalic composition of the initial melt (and bulk rock). The spinels also show strong negative correlations between $\mathrm{Ti}$ and $\mathrm{Mg} /\left(\mathrm{Mg}+\mathrm{Fe}^{2+}\right)$ (Fig. 12) .

The brown spinels in the Hole $801 \mathrm{C}$ tholeiites have a chemistry quite different from those preceding. They are all Ti-poor chrome spinels (criteria of Sigurdsson and Schilling, 1976) with $\mathrm{Cr} /(\mathrm{Cr}+\mathrm{Al}$ ) ratios between 0.191 and $0.380, \mathrm{Mg} /\left(\mathrm{Mg}+\mathrm{Fe}^{2+}\right)$ ratios of $0.567-$ 0.848 , and $\mathrm{Ti}<0.5 \mathrm{cfu}$. Unlike the opaque spinel inclusions described previously, $\mathrm{Ti}$ shows a tendency to increase with $\mathrm{Mg} /\left(\mathrm{Mg}+\mathrm{Fe}^{2+}\right)$ (Fig. 12). There are systematic changes in chemistry between spinel inclusions in plagioclase and olivine and relative to microphenocrysts. Similar variations between spinels in different parageneses have been recorded in MORB younger than the oceanic basalts considered here (Furuta and Tokuyama, 1983; Allan et al., 1988). For example, the $\mathrm{Cr}$ contents of microphenocryst spinels are similar to those of inclusions in plagioclase (5.00-5.55 cfu), but both are richer in $\mathrm{Cr}$ than the inclusions in the olivine microphenocrysts (cf. Furuta and Tokuyama, 1983). $\mathrm{The} \mathrm{Cr} /(\mathrm{Cr}+\mathrm{Al})$ and $\mathrm{Mg} /\left(\mathrm{Mg}+\mathrm{Fe}^{2+}\right)$ ratios for these spinels are similar to chromian spinels from the Mid-Atlantic Ridge picrites (Sigurdsson and Schilling, 1976) and the East Pacific Rise Lamont seamount chain (Allan et al., 1988), although the $\mathrm{Al}$ content exceeds that of many MORB spinels. These features probably reflect the generally primitive nature of the Hole $801 \mathrm{C}$ tholeiites, some of which exhibit high Mg numbers (about 70) not dissimilar to 
Table 5. Amphibole analyses from alkalic dolerites, Holes $800 \mathrm{~A}$ and $802 \mathrm{~A}$.

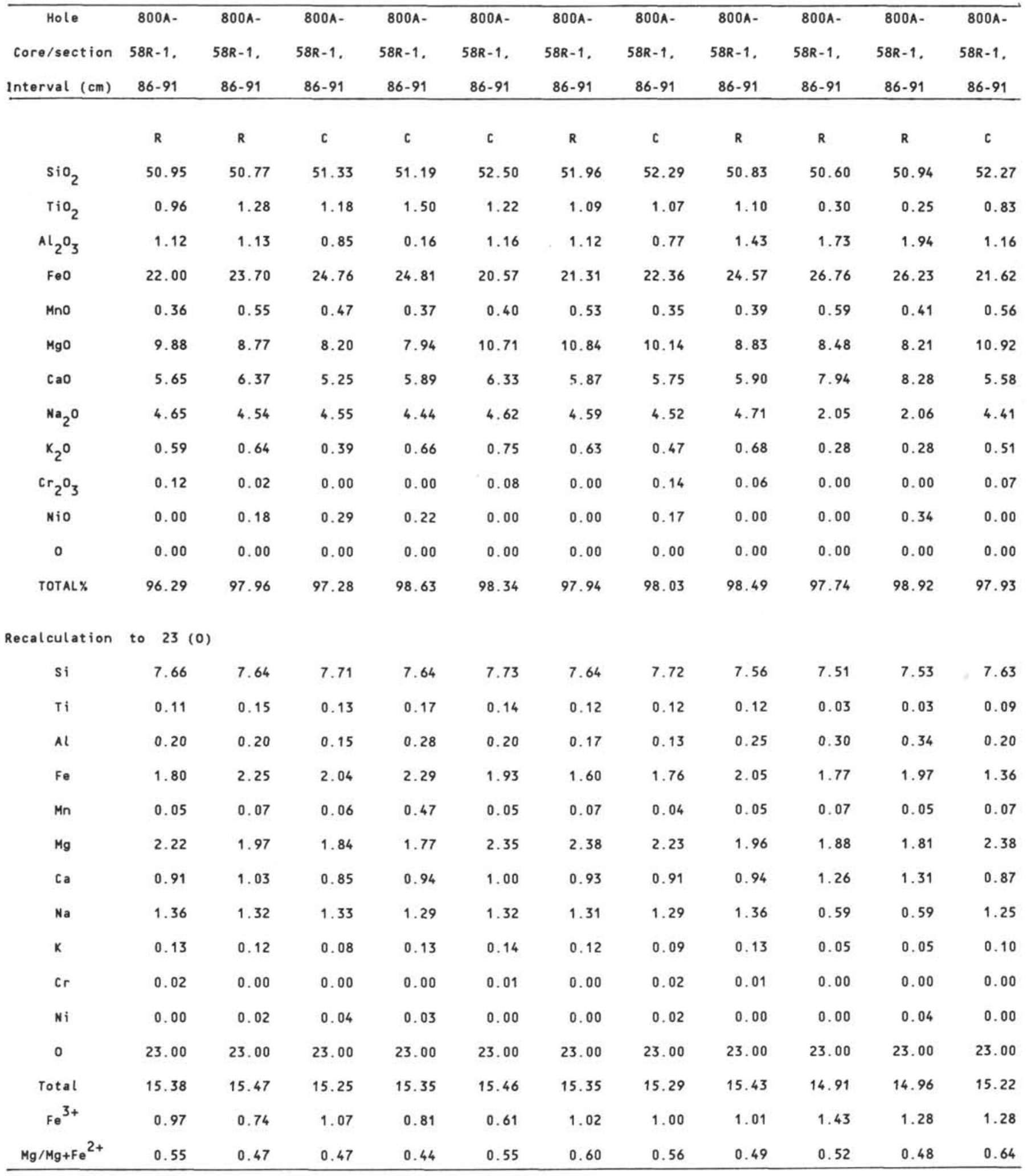

$c=$ Core

$R=\operatorname{Rim}$ 
Table 5 (continued).

\begin{tabular}{|c|c|c|c|c|c|c|c|c|}
\hline $802 A-$ & $802 \mathrm{~A}-$ & $802 \mathrm{~A}-$ & $802 \mathrm{~A}-$ & $802 \mathrm{~A}-$ & $802 \mathrm{~A}-$ & $802 \mathrm{~A}-$ & 802A- & $802 \mathrm{~A}-$ \\
\hline 58R-3. & $58 R-3$ & $58 \mathrm{R}-3$ & $59 R-1$ & $59 R-1$ & $59 R-1$ & $62 R-2$, & $62 R-2$ & $62 R-2$ \\
\hline $90-95$ & $90-95$ & $90-95$ & $138-142$ & $138-142$ & $138-142$ & $53-59$ & $53-59$ & $53-59$ \\
\hline c & $R / C$ & $R$ & c & $R / C$ & $R$ & c & $R / C$ & $R$ \\
\hline 49.68 & 48.32 & 49.44 & 49.04 & 49.45 & 47.95 & 51.11 & 53.36 & 56.83 \\
\hline 0.04 & 0.00 & 0.03 & 0.07 & 0.09 & 0.02 & 0.08 & 0.02 & 0.00 \\
\hline 31.45 & 32.08 & 30.55 & 30.90 & 31.00 & 32.68 & 30.18 & 28.80 & 25.14 \\
\hline 0.85 & 0.65 & 0.65 & 0.93 & 0.79 & 0.67 & 0.77 & 1.03 & 1.22 \\
\hline 0.00 & 0.00 & 0.00 & 0.00 & 0.00 & 0.09 & 0.00 & 0.00 & 0.11 \\
\hline 0.03 & 0.00 & 0.08 & 0.12 & 0.13 & 0.22 & 0.20 & 0.28 & 0.02 \\
\hline 15.86 & 16.71 & 15.40 & 15.60 & 15.72 & 17.04 & 14.43 & 12.79 & 9.85 \\
\hline 2.28 & 2.01 & 2.44 & 2.52 & 2.62 & 1.81 & 3.17 & 4.24 & 5.35 \\
\hline 0.01 & 0.02 & 0.09 & 0.04 & 0.00 & 0.08 & 0.00 & 0.02 & 0.13 \\
\hline 0.06 & 0.10 & 0.00 & 0.00 & 0.04 & 0.01 & 0.08 & 0.00 & 0.06 \\
\hline 0.35 & 0.18 & 0.00 & 0.00 & 0.12 & 0.17 & 0.52 & 0.00 & 0.00 \\
\hline 0.00 & 0.00 & 0.00 & 0.00 & 0.00 & 0.00 & 0.00 & 0.00 & 0.00 \\
\hline 100.59 & 100.07 & 98.68 & 99.23 & 99.94 & 100.72 & 100.53 & 100.53 & 98.70 \\
\hline 2.267 & 2.221 & 2.292 & 2.268 & 2.271 & 2.194 & 2.327 & 2.415 & 2.596 \\
\hline 0.001 & 0.000 & 0.001 & 0.002 & 0.003 & 0.001 & 0.003 & 0.001 & 0.000 \\
\hline 1.691 & 1.738 & 1.670 & 1.685 & 1.678 & 1.762 & 1.620 & 1.536 & 1.353 \\
\hline 0.032 & 0.025 & 0.025 & 0.036 & 0.030 & 0.026 & 0.029 & 0.039 & 0.047 \\
\hline 0.000 & 0.000 & 0.000 & 0.000 & 0.000 & 0.003 & 0.000 & 0.000 & 0.004 \\
\hline 0.002 & 0.000 & 0.006 & 0.009 & 0.009 & 0.015 & 0.013 & 0.019 & 0.001 \\
\hline 0.775 & 0.823 & 0.765 & 0.773 & 0.774 & 0.835 & 0.704 & 0.620 & 0.482 \\
\hline 0.201 & 0.179 & 0.219 & 0.226 & 0.233 & 0.161 & 0.280 & 0.372 & 0.474 \\
\hline 0.001 & 0.001 & 0.006 & 0.003 & 0.000 & 0.004 & 0.000 & 0.001 & 0.007 \\
\hline 0.002 & 0.004 & 0.000 & 0.000 & 0.001 & 0.000 & 0.003 & 0.000 & 0.002 \\
\hline 0.013 & 0.007 & 0.000 & 0.000 & 0.004 & 0.006 & 0.019 & 0.000 & 0.000 \\
\hline 8.000 & 8.000 & 8.000 & 8.000 & 8.000 & 8.000 & 8.000 & 8.000 & 8.000 \\
\hline 4.986 & 4.998 & 4.984 & 5.001 & 5.003 & 5.007 & 4.999 & 5.003 & 4.967 \\
\hline 0.79 & 0.82 & 0.78 & 0.77 & 0.77 & 0.84 & 0.72 & 0.63 & 0.50 \\
\hline
\end{tabular}

primitive seamount compositions (Floyd and Castillo, this volume). This is also substantiated by the inclusion of the spinels in highly magnesian olivines and calcic plagioclases. Overall the $\mathrm{Fe}^{3+}$ contents of the spinels are low and probably indicate that the primitive melts from which the spinels crystallized were extremely reducing $\left(\right.$ low $\mathrm{fO}_{2}$ ). Following the experimental work of Fisk and Bence (1980), a possible sequence of events for the growth of the Hole 801C spinels might be as follows: (1) the individual microphenocrysts of chromian spinel, with some of the highest $\mathrm{Cr} /(\mathrm{Cr}+\mathrm{Al})$ ratios, represent the earliest stage of crystallization and reflect the most primitive melt compositions which have the highest $\mathrm{Mg} /\left(\mathrm{Mg}+\mathrm{Fe}^{2+}\right)$ ratios; (2) as olivine crystallizes the $\mathrm{Cr} /(\mathrm{Cr}+\mathrm{Al})$ ratio decreases in olivine-enclosed spinels, possibly due to a change in $\mathrm{fO}_{2}$ that effects the valency state of the $\mathrm{Cr}$, and (3) as plagioclase begins to crystallize next there is competition for $\mathrm{Al}$ in the melt, such that the $\mathrm{Cr} /(\mathrm{Cr}$ 

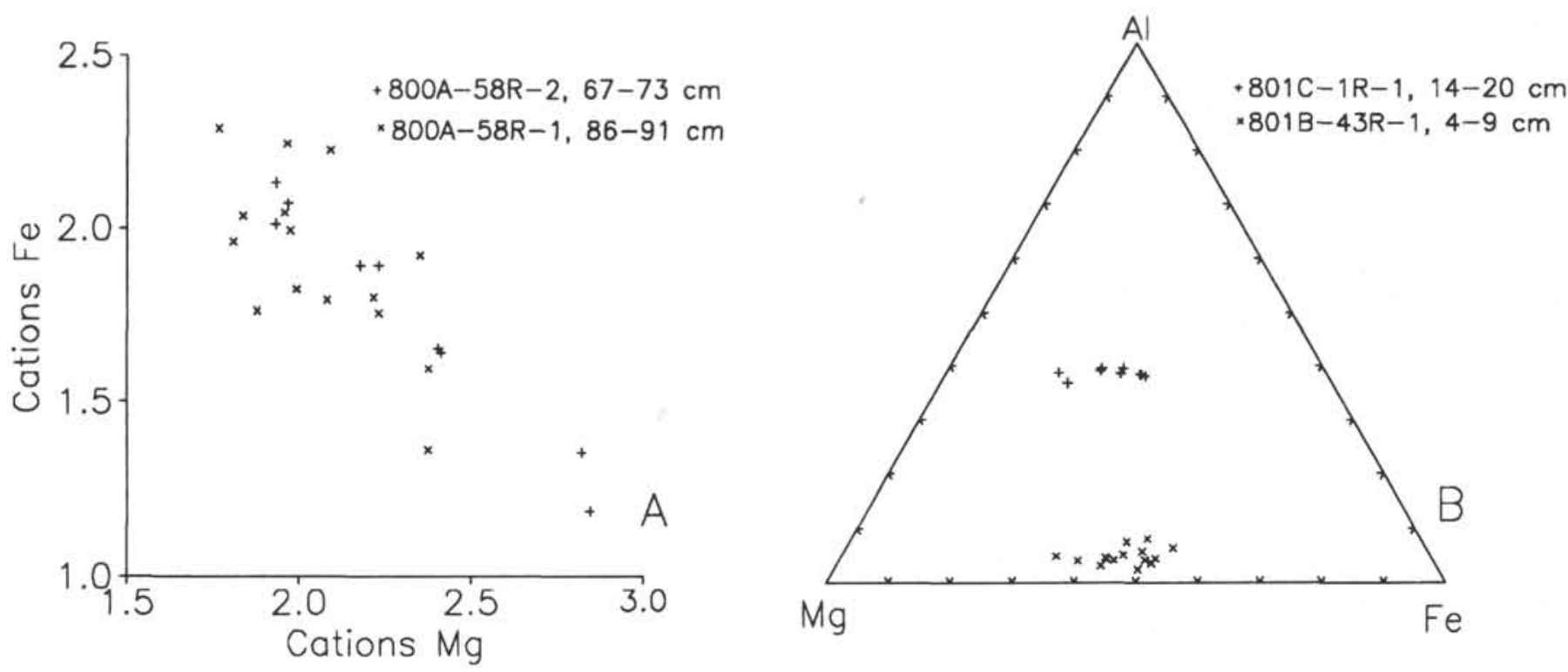

Figure 6. Composition of brown amphiboles from alkalic dolerites and basalts in terms of octahedral components, Al, Mg, and Fe. A. Hole 800A. B. Holes $801 \mathrm{~B}$ and $801 \mathrm{C}$.
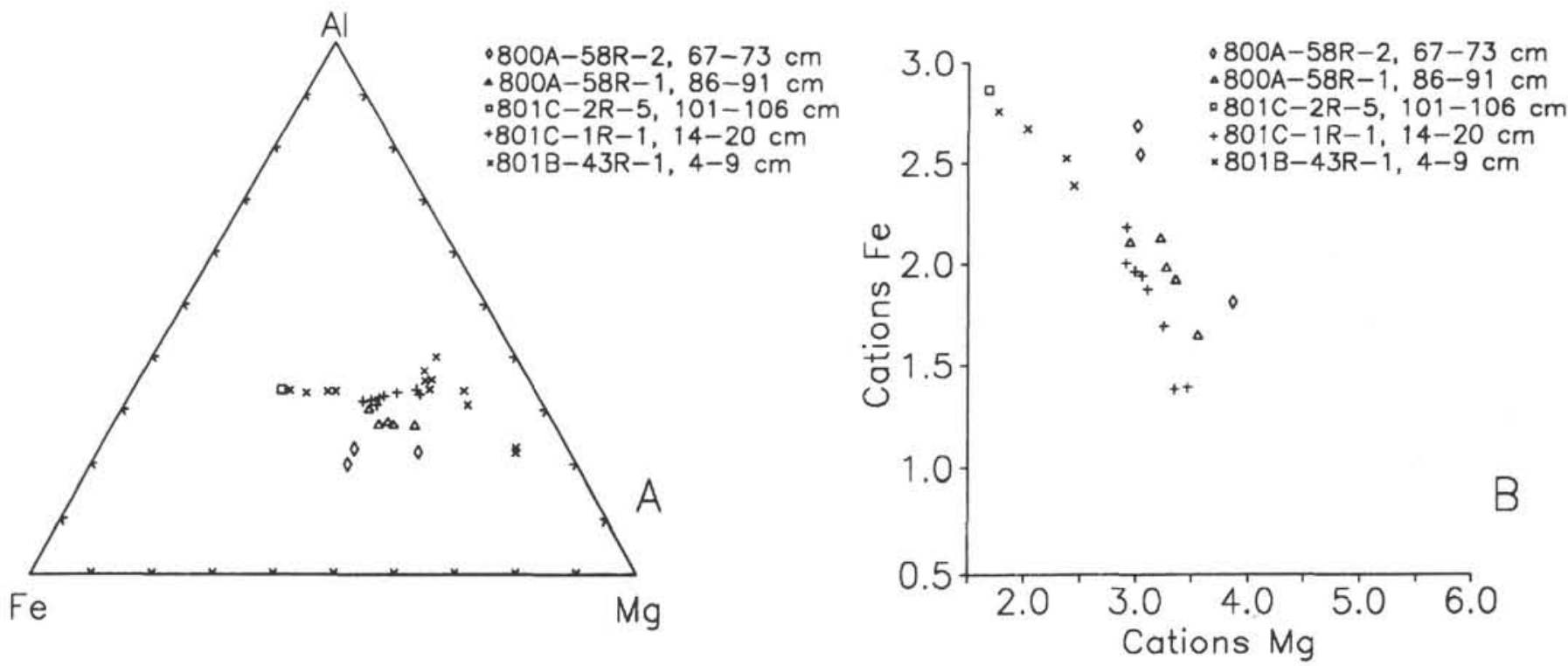

Figure 7. Composition of biotites from alkalic dolerites and basalts in terms of $\mathrm{Al}, \mathrm{Mg}$, and Fe components. A. Holes 800A and 801B. B. Hole 801C.

$+\mathrm{Al}$ ) ratio increases again in spinel inclusions within plagioclase hosts, as the $\mathrm{Mg} /\left(\mathrm{Mg}+\mathrm{Fe}^{2+}\right)$ ratio decreases.

\section{SECONDARY MINERAL PHASE CHEMISTRY}

The majority of the secondary minerals developed in the basaltic and doleritic rocks of Leg 129 are phyllosilicates (smectites and celadonites), together with pyrite, rare zeolite(s), and late replacive carbonate, all occurring within both the bulk rock and veins.

In this section we will be concerned with only the chemical composition of the phyllosilicates (Tables 12-14), which were recalculated on the basis of 22 oxygens in the structural formula. In the accompanying diagrams and tables no account is taken of the presence of ferric iron, as according to Velde (1985) there is no unambiguous method of allocating $\mathrm{Fe}^{3+}$ between octahedral and tetrahedral sites in clay mineral phyllosilicates. Adamson (1981), however, in his study on clay minerals from DSDP Sites 501, 504, and 505, used a recalculation method that determined the $\mathrm{Fe}^{3+}$ content based upon charge-balance requirements in the following formula: $\left(\mathrm{EX}^{+}\right)_{\mathrm{e}}(\mathrm{Al}$, $\left.\mathrm{Fe}^{3+}\right)_{m}\left(\mathrm{Mg}, \mathrm{Fe}^{2+}\right)_{(4+2 t-m)} \mathrm{Si}_{8-5} \mathrm{Al}_{5} \mathrm{O}_{20}(\mathrm{OH})_{4}$.

In the present study, with no $\mathrm{Fe} 3+$ estimation, the total cation addition will be a maximum for that particular analysis. One useful facet (Adamson, 1981) is that by using the total of $\mathrm{Si}+\mathrm{Al}+\mathrm{Mg}+$ $\mathrm{Fe}^{*}$ atoms (SAMF), dioctahedral clays will have a SAMF value of 12 , trioctahedral clays 14 , and mica phyllosilicates 15 . As most clays are mixtures of di- and trioctahedral species, values between the two extremes (12-14) are a function of the interlayering between di- and trioctahedral clays. A recalculation of the structural formula of chlorite from the basis of $28(\mathrm{O})$ to that of $22(\mathrm{O})$ would give a SAMF of 15.7 for a prochlorite composition. As no X-ray powder diffraction analysis was performed on these rocks, there is no independent evidence for the presence of chlorite sensu stricto in these samples. 


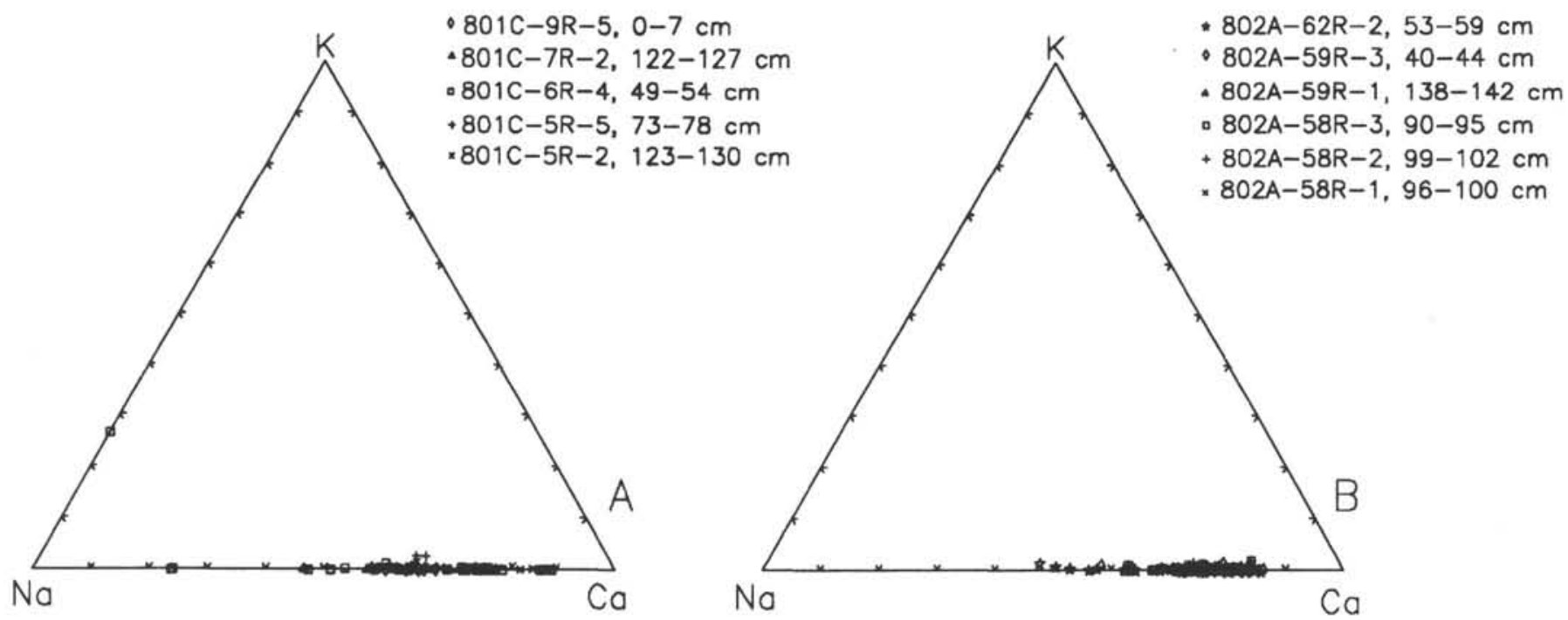

Figure 8. Composition of feldspars from tholeiitic basalt flows, in terms of orthoclase (K), albite (Na), and anorthite (Ca) components. A. Hole 801C. B. Hole $802 \mathrm{~A}$.

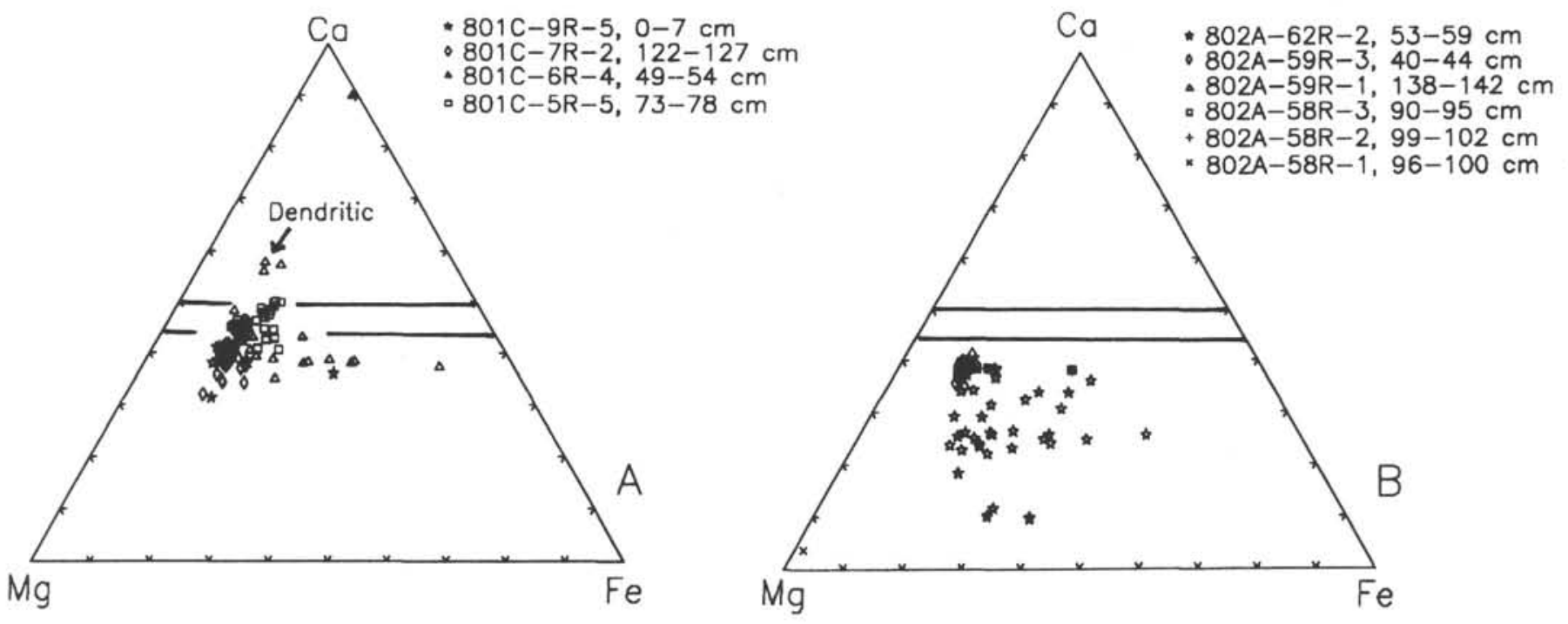

Figure 9. Composition of clinopyroxenes from tholeiitic basalt flows, in terms of Ca, Fe, and Mg. A. Hole 801C. B. Hole 802A.

\section{Phyllosilicates in Alkalic Dolerites and Basalts}

The phyllosilicates analyzed in the Hole $800 \mathrm{~A}$ alkaline dolerites occur either as a replacement phase of pseudohexagonal plates of very late biotite (bright green coloration) or as alteration patches (brownish olive green colored masses) within the matrix and mesostasis. The bright green variety is relatively $\mathrm{Al}$ - and $\mathrm{Fe}$-rich with $\mathrm{Fe} / \mathrm{Mg}$ ratios up to 0.825 and an SAMF value of about 15. The brownish olive green species with lower $\mathrm{Fe} / \mathrm{Mg}$ ratios of 0.306 has an SAMF value of about 14. The interlayer cations of both varieties have $\mathrm{Na}>\mathrm{K}<\mathrm{Ca}$ (Fig. 13B). Using the criteria of Adamson (1981), the bright green phyllosilicate is a chlorite-smectite rather than a true chlorite, as it possesses significant interlayer cation occupancy. The brownish olive green variety is a smectite with significant $\mathrm{Fe}-\mathrm{Mg}$ occupancy and is a member of the saponite-ferrosaponite series of trioctahedral smectites. True chlorites, together with di- and trioctahedral smectites, have been recorded in deeper oceanic basement sequences such as at Sites 501, 504, and 505 (Adamson, 1981; Alt et al., 1985).
The alteration pattern in the alkalic basalts of Holes $801 \mathrm{~B}$ and $801 \mathrm{C}$ is similar to that in Hole 800A dolerites. In Sample 801C-1R-1, 14-20 $\mathrm{cm}$, for example, there are three varieties of phyllosilicate: a fine-grained amorphous brown variety found as interstitial patches throughout the rock, a green fibrous mineral that nucleates on either microfractures in primary minerals or earlier clay minerals, and platelets that pseudomorph olivine. All three varieties have a different chemistry, which in part reflects the composition of the host phase. The mineral commonly replacing olivine is a Mg-saponite close to the boundary with the talc-pyrophyllite series that is $\mathrm{Al}$-poor $(0.34-0.48 \mathrm{cfu})$ with low $\mathrm{Fe} / \mathrm{Mg}$ ratio (0.356) and an SAMF of about 14.00. The brown amorphous clay and the green fibers have lower $\mathrm{Fe} / \mathrm{Mg}$ ratios ( 0.310 and 0.247 , respectively) and higher, but different, $\mathrm{Al}$ contents ( 0.80 and $1.52 \mathrm{cfu}$, respectively). All three have an SAMF of about 14.00 and are therefore saponites with variable proportions of $\mathrm{Al}$ in tetrahedral coordination. Other greenish clays, however (for example, Sample 801B-43R-1,4-9 cm), are highly aluminous (3.64 cfu), with low Fe/Mg (0.33) and SAMF values of about 15.00, and are chlorite-smectite, similar to those in the alkalic dolerites (Table 12). 
Table 6. Biotite analyses from alkalic dolerites and basalts, Holes 800A, 801B, and 801C.

\begin{tabular}{|c|c|c|c|c|c|c|c|c|c|c|c|}
\hline Hole & $800 \mathrm{~A}-$ & $800 \mathrm{~A}$ - & $800 \mathrm{~A}$ - & $800 \mathrm{~A}-$ & $800 \mathrm{~A}-$ & $800 \mathrm{~A}$ - & $800 \mathrm{~A}-$ & 800A- & $801 \mathrm{C}$ - & $801 \mathrm{C}$ - & 801C- \\
\hline Core/section & $58 R-1$ & $58 R-1$ & $58 R-1$ & $58 R-1$ & $58 R-1$ & $58 R-1$ & $58 R-1$ & $58 R-1$ & $1 R-1$ & $1 R=1$ & $1 R-1$ \\
\hline \multirow[t]{2}{*}{ Interval $(\mathrm{cm})$} & $86-91$ & $86-91$ & 86.91 & $86-91$ & $67-73$ & $67-73$ & $67-73$ & $67-73$ & $14-20$ & $14-20$ & $14-20$ \\
\hline & $\mathbf{R}$ & R & c & c & $\mathbf{R}$ & c & c & R & R & c & c \\
\hline $\mathrm{SiO}_{2}$ & 40.02 & 39.67 & 39.42 & 39.01 & 40.47 & 41.81 & 41.90 & 37.83 & 36.61 & 36.39 & 36.75 \\
\hline $\mathrm{TiO}_{2}$ & 5.09 & 5.23 & 5.07 & 5.12 & 3.08 & 2.99 & 3.02 & 4.82 & 7.38 & 5.09 & 7.36 \\
\hline $\mathrm{Al}_{2} \mathrm{O}_{3}$ & 11.65 & 11.07 & 10.96 & 11.50 & 8.12 & 9.57 & 10.22 & 12.18 & 13.77 & 13.78 & 14.38 \\
\hline $\mathrm{FeO}$ & 12.87 & 13.32 & 15.52 & 16.04 & 21.10 & 17.89 & 14.30 & 19.15 & 11.26 & 14.14 & 13.86 \\
\hline Mno & 0.00 & 0.00 & 0.13 & 0.15 & 0.10 & 0.00 & 0.05 & 0.13 & 0.01 & 0.32 & 0.06 \\
\hline $\mathrm{MgO}$ & 16.65 & 16.08 & 14.99 & 14.81 & 13.26 & 15.35 & 17.40 & 12.40 & 15.65 & 15.02 & 14.85 \\
\hline $\mathrm{CaO}$ & 0.08 & 0.00 & 0.00 & 0.33 & 0.00 & 0.01 & 0.08 & 0.12 & 0.14 & 0.34 & 0.18 \\
\hline $\mathrm{Na}_{2} \mathrm{O}$ & 1.22 & 1.11 & 0.86 & 1.00 & 0.69 & 0.85 & 1.04 & 0.85 & 1.20 & 0.89 & 1.02 \\
\hline $\mathrm{K}_{2} \mathrm{O}$ & 8.67 & 8. 51 & 8.42 & 8.48 & 8. 12 & 8.67 & 8.15 & 8.67 & 8.17 & 7.92 & 7.67 \\
\hline $\mathrm{Cr}_{2} \mathrm{O}_{3}$ & 0.04 & 0.01 & 0.00 & 0.00 & 0.07 & 0.08 & 0.02 & 0,10 & 0.03 & 0.00 & 0.05 \\
\hline NiO & 0.00 & 0.00 & 0.23 & 0.10 & 0.17 & 0.49 & 0.18 & 0.28 & 0.33 & 0.00 & 0.00 \\
\hline TOTAL\% & 96.30 & 95.02 & 95.59 & 96.53 & 95.17 & 97.70 & 96.35 & 96.53 & 94.52 & 93.88 & 96.17 \\
\hline \multicolumn{12}{|c|}{ Recalculation to $22(0)$} \\
\hline si & 5.84 & 5.88 & 5.87 & 5.78 & 6.21 & 6.14 & 6.11 & 5.71 & 5.43 & 5.50 & 5.39 \\
\hline Ti & 0.56 & 0.58 & 0.57 & 0.57 & 0.36 & 0.33 & 0.33 & 0.55 & 0.82 & 0.58 & 0.81 \\
\hline AL & 2.01 & 1.93 & 1.93 & 2. 01 & 1.47 & 1.66 & 1.76 & 2.17 & 2.41 & 2.46 & 2.49 \\
\hline $\mathrm{Fe}$ & 1.57 & 1.65 & 1.93 & 1.99 & 2.71 & 2.20 & 1.75 & 2.42 & 1.40 & 1.79 & 1.70 \\
\hline Mn & 0.00 & 0.00 & 0.02 & 0.02 & 0.01 & 0.00 & 0.01 & 0.02 & 0.00 & 0.04 & 0.01 \\
\hline Mg & 3.63 & 3.55 & 3.33 & 3.27 & 3.03 & 3.36 & 3.78 & 2.79 & 3.46 & 3.38 & 3.25 \\
\hline $\mathrm{Ca}$ & 0.01 & 0.00 & 0.00 & 0.05 & 0.00 & 0.00 & 0.01 & 0.02 & 0.02 & 0.05 & 0.03 \\
\hline $\mathrm{Na}$ & 0.34 & 0.32 & 0.25 & 0.29 & 0.20 & 0.24 & 0.29 & 0.25 & 0.35 & 0.26 & 0.29 \\
\hline$k$ & 1.62 & 1.61 & 1.60 & 1.60 & 1.59 & 1.63 & 1.52 & 1.67 & 1.55 & 1.53 & 1.44 \\
\hline $\mathrm{Cr}$ & 0.01 & 0.00 & 0.00 & 0.00 & 0.01 & 0.01 & 0.00 & 0.01 & 0.00 & 0.00 & 0.01 \\
\hline $\mathrm{Ni}$ & 0.00 & 0.00 & 0.03 & 0.01 & 0.02 & 0.06 & 0.02 & 0.03 & 0.04 & 0.00 & 0.00 \\
\hline 0 & 22.00 & 22.00 & 22.00 & 22.00 & 22.00 & 22.00 & 22.00 & 22.00 & 22.00 & 22.00 & 22.00 \\
\hline Total & 15.57 & 15.53 & 15.49 & 15.58 & 15.57 & 15.56 & 15.56 & 15.57 & 15.44 & 15.59 & 15.40 \\
\hline $\mathrm{Mg} / \mathrm{Mg}+\mathrm{Fe}$ & 0.70 & 0.68 & 0.63 & 0.62 & 0.53 & 0.60 & 0.68 & 0.54 & 0.71 & 0.65 & 0.66 \\
\hline
\end{tabular}

Footnote:

$\begin{array}{lll}\text { C } & =\quad \text { Core } \\ \text { R } & =\quad \text { Rim }\end{array}$

Bright green celadonite occurs with smectites in Sample 801C-2R$5,101-106 \mathrm{~cm}$, replacing mesostasis. The interlayer charge is about $1.80, \mathrm{Ex}^{+}$is largely $\mathrm{K}^{+}$, tetrahedral sites are filled by $\mathrm{Si}$, and octahedral cation proportions are $\mathrm{Al}>\mathrm{Mg}>\mathrm{Fe}$, which is unusual for celadonites. The smectite that occurs with this celadonite has a similar octahedral site occupancy and $\mathrm{Fe} / \mathrm{Mg}$ ratio $(0.555$, compared to 0.672 in the celadonite), and an SAMF of about 13.00, denoting that the interlay- ering is approximately 50:50 di- to trioctahedral smectite. Similarity in octahedral cation occupancy appears to be a characteristic of coexisting smectites and celadonites in basaltic rocks and may be a function of the replacement of early smectites by later celadonites. Fig. 13A (modified from Velde, 1985) shows that divalent cations dominate the octahedral sites $\left(3 \mathrm{R}^{2+}\right)$ with K-rich celadonite compositions separated from smectites that display highly variable $\mathrm{Na}$ and $\mathrm{Ca}$ distributions. 
Table 6 (continued).

\begin{tabular}{|c|c|c|c|c|c|c|}
\hline $801 \mathrm{C}$ - & $801 \mathrm{C}$ - & $801 \mathrm{C}-$ & $801 \mathrm{C}-$ & 8016 . & 8018 - & 8018- \\
\hline $1 R-1$ & $1 R-1$ & $1 R-1$ & $1 R-1$ & $1 R-1$ & $44 R-2$ & $44 R-2$ \\
\hline $14-20$ & $14-20$ & $14-20$ & $14-20$ & $14-20$ & $140-145$ & $140-145$ \\
\hline$E / R$ & $R$ & $R$ & $E / R$ & $E / R$ & $R$ & $E / R$ \\
\hline 50.57 & 50.44 & 50.44 & 51.10 & 50.97 & 50.74 & 50.88 \\
\hline 4.46 & 4.28 & 4.28 & 4.47 & 4.87 & 5.11 & 3.97 \\
\hline 0.45 & 0.64 & 0.64 & 0.64 & 0.69 & 2.06 & 2.39 \\
\hline 19.27 & 10.99 & 10.99 & 14.14 & 13.47 & 20.67 & 22.65 \\
\hline 6.75 & 14.24 & 14.24 & 11.70 & 11.83 & 4.74 & 3.32 \\
\hline 0.29 & 0.81 & 0.81 & 0.43 & 0.52 & 0.30 & 0.25 \\
\hline 0.23 & 0.35 & 0.35 & 0.19 & 0.17 & 1.80 & 2.20 \\
\hline 6.51 & 11.16 & 11.16 & 8.52 & 8.33 & 2.01 & 1.90 \\
\hline 10.48 & 7.37 & 7.37 & 9.00 & 9.05 & 11.72 & 11.75 \\
\hline 0.03 & 0.00 & 0.00 & 0.00 & 0.01 & 0.03 & 0.00 \\
\hline 99.04 & 100.28 & 100.28 & 100.19 & 99.91 & 99.18 & 99.31 \\
\hline 1.97 & 1.98 & 1.98 & 1.99 & 1.99 & 1.94 & 1.94 \\
\hline 0.13 & 0.13 & 0.13 & 0.13 & 0.14 & 0.15 & 0.11 \\
\hline 0.02 & 0.03 & 0.03 & 0.03 & 0.03 & 0.09 & 0.11 \\
\hline 0.57 & 0.32 & 0.32 & 0.41 & 0.40 & 0.60 & 0.65 \\
\hline 0.22 & 0.47 & 0.47 & 0.38 & 0.39 & 0.15 & 0.11 \\
\hline 0.01 & 0.03 & 0.03 & 0.01 & 0.02 & 0.01 & 0.01 \\
\hline 0.01 & 0.02 & 0.02 & 0.01 & 0.01 & 0.10 & 0.13 \\
\hline 0.27 & 0.47 & 0.47 & 0.36 & 0.35 & 0.08 & 0.13 \\
\hline 0.79 & 0.56 & 0.56 & 0.68 & 0.68 & 0.87 & 0.08 \\
\hline 0.00 & 0.00 & 0.00 & 0.00 & 0.00 & 0.00 & 0.87 \\
\hline 6.00 & 6.00 & 6.00 & 6.00 & 6.00 & 6.00 & 6.00 \\
\hline 4.00 & 4.00 & 4.00 & 4.00 & 4.00 & 4.00 & 4.00 \\
\hline
\end{tabular}

\section{Phyllosilicates in Tholeiitic Basalts}

There are many similarities between the chemistry of the secondary phyllosilicates in the alkali basalts (Fig. 13) and those in the tholeiitic basalts (Figs. 14 and 15) with both smectites (of variable composition) and celadonites present (Tables 13 and 14, respectively). Although smectite analyses from the same sample tend to group close together, part of the spread reflects the nature of the replaced host, for example, olivine relative to glassy mesostasis.

Color variation in smectites from Site 801 tholeiites is common, although most of the yellow-brown and dark brown varieties replacing interstitial material are intermediate tri- and dioctahedral smectites with SAMF values of about 13. The dark brown varieties are invariably more $\mathrm{Mg}$-and $\mathrm{Al}$-rich than the yellow-brown species, with distinct $\mathrm{Fe} / \mathrm{Mg}$ ratios ( 0.720 and 1.050 respectively). The dominant greenish phyllosilicates of Hole $801 \mathrm{C}$ below the hydrothermal deposit are Fe-rich saponites and celadonites (Tables 13 and 14).

Phyllosilicates analyzed in the Hole $802 \mathrm{~A}$ hypocrystalline tholeiitic basalts are almost exclusively interstitial and represent the replacement of original glass, although smectite pseudomorphs after olivine occur in Sample 802 A-58R-2, 99-102 cm. The smectites are all highly siliceous (the majority of the analyses yield $\mathrm{Si}>7.00 \mathrm{cfu}$; Table 10) and recalculate to a saponite-ferrosaponite formula (Guven, 1988), that is, $\mathrm{Ex}+(\mathrm{Mg}-\mathrm{Fe})_{6}\left(\mathrm{Si}_{8}-x \mathrm{Al}\right) 0_{20}(\mathrm{OH})_{4}$, where $\mathrm{Ex}^{+}$represents the interlayer cation. Figure $15 \mathrm{~B}$ demonstrates the mutual replacement of $\mathrm{Mg}$ by $\mathrm{Fe}$ in the smectites, and the clustering of data for specific samples suggests that their composition is influenced by bulk-rock $\mathrm{Fe} / \mathrm{Mg}$ ratios. In general, saponite $\mathrm{Fe} / \mathrm{Mg}$ ratios from replaced glass are lower and more variable (about $0.43-0.65$ ) than those replacing olivine (0.77). Al substitution for $\mathrm{Si}$ in the tetrahedral sites is extremely limited (Fig. 15) where the Si values tightly group between 7.0 and $7.5 \mathrm{cfu}$. The excess of $\mathrm{Al}$ over that required to fill the tetrahedral sites must indicate that there is limited solid solution either toward chlorite compositions or di-octahedral smectites of beidellite composition. The preponderance of divalent over trivalent cations is demonstrated in Figure 15A, where a tight clustering of smectite compositions is again observed. The total interlayer cation content of the saponites never exceeds $0.60 \mathrm{cfu}$, of which the dominant interlayer cation is $\mathrm{Ca}$ (usually $0.2-0.3 \mathrm{cfu}$ ) with smaller contents of $\mathrm{Na}$ and $\mathrm{K}$ (Fig. 15C). All the analyses have an SAMF of $13-14$ and total interlayer cations $<0.6$, and are therefore dominantly trioctahedral smectites or saponites.

The Hole 802A celadonites (Fig. 15 and Table 14) occur in interstitial patches and have recalculated $\mathrm{Si}$ values $>8.00$ and $\mathrm{Al}$ about $0.50 \mathrm{cfu}$. In comparison to the saponite smectites the $\mathrm{Fe} / \mathrm{Mg}$ ratio in the celadonites is higher and more varied, ranging between 1.569 (Sample 802A-58R-2, 99-102 cm) and 3.896 (Sample 802A$59 \mathrm{R}-1,138-142 \mathrm{~cm}$ ). The interlayer cation is almost exclusively $\mathrm{K}$ with only minor amounts of $\mathrm{Na}$ and $\mathrm{Ca}$.

\section{Carbonates in Tholeiitic Basalts}

Carbonates, intimately associated with phyllosilicates, are present in many of the alkalic and tholeiitic basalts, replacing matrix and individual phases, and also commonly occur as a fracture vein infilling. In Hole $801 \mathrm{C}$, vein carbonate associated with celadonite (for example, Sample 801 C-5R-3, 125-131 cm) exhibits layers deposited in a sequential manner with granular carbonate material adjacent to the walls and coarser rhombic crystals in the vein interior. Individual layers show considerable variation in terms of the molecular proportions of $\mathrm{Ca}, \mathrm{Mg}$, and $\mathrm{Fe}$ carbonate end-members (Table 15) and imply that the fluids passing through the vein had highly variable compositions, initially $\mathrm{Mg}$ and Fe-rich relative to $\mathrm{Ca}$. The carbonates vary from ferroan dolomites to sideritic carbonates to calcite, with the latter forming the last phase to crystallize in the vein interior.

\section{SUMMARY AND CONCLUSIONS}

In this section we briefly summarize the main compositional variations of the primary and secondary phases in Leg 129 basaltic rocks and relate systematic chemical changes in the major magmatic silicates to simple petrogenetic schemes.

\section{Alkalic Dolerites and Basalts}

The alkalic dolerite sills (Hole 800A) and alkalic basalt flows (Site 801 ) are similar petrographically, but differ slightly in their relative degrees of chemical fractionation and levels of incompatible trace elements. Both the sills and lava flows have evolved basic compositions (commonly hawaiites), which are also reflected in the composition of the feldspars (with labradorite cores), presence of abundant titanomagnetite, and late-stage hydrous phases. 
G. ROWBOTHAM, P.A. FLOYD

Table 7. Plagioclase analyses from tholeiitic basalts, Holes $801 \mathrm{C}$ and $802 \mathrm{~A}$.

\begin{tabular}{|c|c|c|c|c|c|c|c|c|c|c|c|}
\hline Hole & $801 \mathrm{C}$ - & $801 \mathrm{C}$ - & $801 \mathrm{C}$ - & $801 \mathrm{C}$ - & $801 \mathrm{C}$. & $801 \mathrm{C}$ - & $801 \mathrm{C}-$ & $801 \mathrm{C}-$ & $801 C-$ & $802 A-$ & 802A- \\
\hline Core/section & $5 R-2$ & $5 R-2$. & $5 R-2$ & $6 R-4$ & $6 R-4$ & $6 R-4$. & $6 R-4$. & $9 R-5$. & $9 R-5$ & $58 R-2$ & $58 R-2$ \\
\hline Interval (cm) & $123-130$ & $123-130$ & $123-130$ & $49-54$ & $49-54$ & 49.54 & $49-54$ & $0-7$ & $0-7$ & $99-102$ & $99-102$ \\
\hline & c & R & 6 & c & $s$ & s & c & c & $\mathbf{R}$ & c & R \\
\hline $\mathrm{SiO}_{2}$ & 47.98 & 49.04 & 52.52 & 44.92 & 50.87 & 61.81 & 46.20 & 52.32 & 60.66 & 51.43 & 49.27 \\
\hline $\mathrm{TiO}_{2}$ & 0.08 & 0.00 & 0.13 & 0.09 & 0.21 & 0.19 & 0.03 & 0.06 & 0.10 & 0.00 & 0.06 \\
\hline $\mathrm{Al}_{2} \mathrm{O}_{3}$ & 33.14 & 31.87 & 27.45 & 34.10 & 28.10 & 22.42 & 33.55 & 29.33 & 23.14 & 30.23 & 31.01 \\
\hline $\mathrm{FeO}$ & 0.47 & 0.47 & 1.91 & 0.35 & 1.24 & 1.39 & 0.20 & 0.82 & 1.02 & 0.79 & 0.81 \\
\hline Mno & 0.09 & 0.00 & 0.00 & 0.00 & 0.01 & 0.00 & 0.00 & 0.00 & 0.00 & 0.00 & 0.10 \\
\hline $\mathrm{MgO}$ & 0.00 & 0.00 & 0.75 & 0.28 & 0.33 & 0.00 & 0.08 & 0.14 & 0.19 & 0.32 & 0.20 \\
\hline $\mathrm{CaO}$ & 17.14 & 16.07 & 12.78 & 18.02 & 12.94 & 4.87 & 17.78 & 13.45 & 6.47 & 14.60 & 15.80 \\
\hline $\mathrm{Na}_{2} \mathrm{O}$ & 1.84 & 2.32 & 3.61 & 1.10 & 3.95 & 8.29 & 1.28 & 3.79 & 7.38 & 3.25 & 2.63 \\
\hline $\mathrm{K}_{2} \mathrm{O}$ & 0.01 & 0.00 & 0.27 & 0.00 & 0.00 & 0.13 & 0.03 & 0.00 & 0.06 & 0.00 & 0.05 \\
\hline $\mathrm{Cr}_{2} \mathrm{O}_{3}$ & 0.00 & 0.16 & 0.10 & 0.00 & 0.00 & 0.06 & 0.01 & 0.09 & 0.00 & 0.08 & 0.03 \\
\hline NiO & 0.02 & 0.00 & 0.22 & 0.25 & 0.11 & 0.09 & 0.00 & 0.12 & 0.15 & 0.00 & 0.16 \\
\hline 0 & 0.00 & 0.00 & 0.00 & 0.00 & 0.00 & 0.00 & 0.00 & 0.00 & 0.00 & 0.00 & 0.00 \\
\hline TOTAL \% & 100.78 & 99.94 & 99.75 & 99.10 & 97.74 & 99.26 & 99.14 & 100.11 & 99.19 & 100.71 & 100.11 \\
\hline Recalculation & to $8(0)$ & & & & & & & & & & \\
\hline si & 2.19 & 2.25 & 2.41 & 2.10 & 2.38 & 2.78 & 2.14 & 2.38 & 2.73 & 2.33 & 2.26 \\
\hline$T i$ & 0.00 & 0.00 & 0.00 & 0.00 & 0.01 & 0.01 & 0.00 & 0.00 & 0.00 & 0.00 & 0.00 \\
\hline Al & 1.78 & 1.72 & 1.49 & 1.87 & 1.55 & 1.19 & 1.84 & 1.57 & 1.23 & 1.62 & 1.68 \\
\hline $\mathrm{Fe}$ & 0.02 & 0.02 & 0.07 & 0.01 & 0.05 & 0.05 & 0.01 & 0.03 & 0.04 & 0.03 & 0.03 \\
\hline $\mathrm{Mn}$ & 0.00 & 0.00 & 0.00 & 0.00 & 0.00 & 0.00 & 0.00 & 0.00 & 0.00 & 0.00 & 0.00 \\
\hline $\mathrm{Mg}$ & 0.00 & 0.00 & 0.05 & 0.02 & 0.02 & 0.00 & 0.01 & 0.01 & 0.01 & 0.02 & 0.01 \\
\hline $\mathrm{Ca}$ & 0.84 & 0.79 & 0.63 & 0.90 & 0.65 & 0.24 & 0.88 & 0.66 & 0.31 & 0.71 & 0.78 \\
\hline $\mathrm{Na}$ & 0.16 & 0.21 & 0.32 & 0.10 & 0.36 & 0.73 & 0.12 & 0.33 & 0.64 & 0.29 & 0.23 \\
\hline$k$ & 0.00 & 0.00 & 0.02 & 0.00 & 0.00 & 0.01 & 0.00 & 0.00 & 0.00 & 0.00 & 0.00 \\
\hline $\mathrm{Cr}$ & 0.00 & 0.01 & 0.00 & 0.00 & 0.00 & 0.00 & 0.00 & 0.00 & 0.00 & 0.00 & 0.00 \\
\hline $\mathrm{Ni}$ & 0.00 & 0.00 & 0.01 & 0.01 & 0.00 & 0.00 & 0.00 & 0.00 & 0.01 & 0.00 & 0.01 \\
\hline 0 & 8.00 & 8.00 & 8.00 & 8.00 & 8.00 & 8.00 & 8.00 & 8.00 & 8.00 & 8.00 & 8.00 \\
\hline Total & 5.00 & 4.99 & 5.01 & 5.01 & 5.02 & 4.99 & 4.99 & 4.99 & 4.98 & 5.00 & 5.01 \\
\hline $\mathrm{Ca} / \mathrm{Ca}+\mathrm{Na}$ & 0.84 & 0.79 & 0.66 & 0.90 & 0.64 & 0.24 & 0.88 & 0.66 & 0.33 & 0.71 & 0.77 \\
\hline
\end{tabular}

Footnote:

$c=$ Core

$R=R i m$

$R / C=$ Intermediate core-rim

$G=$ Groundmass

$S=$ Spherulitic groundmass 
Table 7 (continued).

\begin{tabular}{|c|c|c|c|c|c|c|c|c|}
\hline $802 A-$ & 802A- & 802A- & 802A- & 802A- & $802 A-$ & $802 A-$ & 802A- & 802A- \\
\hline $58 R-3$ & $58 R-3$ & $58 \mathrm{R}-3$ & $59 R-1$. & $59 R-1$. & $59 R-1$ & $62 \mathrm{R}-2$ & $62 R-2$ & $62 R-2$ \\
\hline $90-95$ & $90-95$ & $90-95$ & $138-142$ & $138-142$ & $138-142$ & 53.59 & 53.59 & $53-59$ \\
\hline c & $R / C$ & R & c & $R / C$ & $R$ & c & $R / C$ & $R$ \\
\hline 49.68 & 48.32 & 49.44 & 49.04 & 49.45 & 47.95 & 51.11 & 53.36 & 56.83 \\
\hline 0.04 & 0.00 & 0.03 & 0.07 & 0.09 & 0.02 & 0.08 & 0.02 & 0.00 \\
\hline 31.45 & 32.08 & 30.55 & 30.90 & 31.00 & 32.68 & 30.18 & 28.80 & 25.14 \\
\hline 0.85 & 0.65 & 0.65 & 0.93 & 0.79 & 0.67 & 0.77 & 1.03 & 1.22 \\
\hline 0.00 & 0.00 & 0.00 & 0.00 & 0.00 & 0.09 & 0.00 & 0.00 & 0.11 \\
\hline 0.03 & 0.00 & 0.08 & 0.12 & 0.13 & 0.22 & 0.20 & 0.28 & 0.02 \\
\hline 15.86 & 16.71 & 15.40 & 15.60 & 15.72 & 17.04 & 14.43 & 12.79 & 9.85 \\
\hline 2.28 & 2.01 & 2.44 & 2.52 & 2.62 & 1.81 & 3.17 & 4.24 & 5.35 \\
\hline 0.01 & 0.02 & 0.09 & 0.04 & 0.00 & 0.08 & 0.00 & 0.02 & 0.13 \\
\hline 0.06 & 0.10 & 0.00 & 0.00 & 0.04 & 0.01 & 0.08 & 0.00 & 0.06 \\
\hline 0.35 & 0.18 & 0.00 & 0.00 & 0.12 & 0.17 & 0.52 & 0.00 & 0.00 \\
\hline 0.00 & 0.00 & 0.00 & 0.00 & 0.00 & 0.00 & 0.00 & 0.00 & 0.00 \\
\hline 100.59 & 100.07 & 98.68 & 99.23 & 99.94 & 100.72 & 100.53 & 100.53 & 98.70 \\
\hline 2.27 & 2.22 & 2.29 & 2.27 & 2.27 & 2.19 & 2.33 & 2.42 & 2.60 \\
\hline 0.00 & 0.00 & 0.00 & 0.00 & 0.00 & 0.00 & 0.00 & 0.00 & 0.00 \\
\hline 1.69 & 1.74 & 1.67 & 1.69 & 1.68 & 1.76 & 1.62 & 1.54 & 1.35 \\
\hline 0.03 & 0.03 & 0.03 & 0.04 & 0.03 & 0.03 & 0.03 & 0.04 & 0.05 \\
\hline 0.00 & 0.00 & 0.00 & 0.00 & 0.00 & 0.00 & 0.00 & 0.00 & 0.00 \\
\hline 0.00 & 0.00 & 0.01 & 0.01 & 0.01 & 0.02 & 0.01 & 0.02 & 0.00 \\
\hline 0.78 & 0.82 & 0.77 & 0.77 & 0.77 & 0.84 & 0.70 & 0.62 & 0.48 \\
\hline 0.20 & 0.18 & 0.22 & 0.23 & 0.23 & 0.16 & 0.28 & 0.37 & 0.47 \\
\hline 0.00 & 0.00 & 0.01 & 0.00 & 0.00 & 0.00 & 0.00 & 0.00 & 0.01 \\
\hline 0.00 & 0.00 & 0.00 & 0.00 & 0.00 & 0.00 & 0.00 & 0.00 & 0.00 \\
\hline 0.01 & 0.01 & 0.00 & 0.00 & 0.00 & 0.01 & 0.02 & 0.00 & 0.00 \\
\hline 8.00 & 8.00 & 8.00 & 8.00 & 8.00 & 8.00 & 8.00 & 8.00 & 8.00 \\
\hline 4.99 & 5.00 & 4.98 & 5.00 & 5.00 & 5.01 & 5.00 & 5.00 & 4.97 \\
\hline 0.79 & 0.82 & 0.78 & 0.77 & 0.77 & 0.84 & 0.72 & 0.63 & 0.50 \\
\hline
\end{tabular}

Although erupted under different conditions, both groups of alkalic rocks show a number of similar chemical features.

1. The feldspars are commonly zoned with calcic cores (commonly labradorite in Hole 800A; labradorite-bytownite at Site 801) to sodic rims that invariably have a high orthoclase component, such that many rim zones are essentially alkali feldspars (maximum up to $\mathrm{Or}_{69}$ ). Apart from normal zoning, oscillatory zoning may be present (again labradorite core to alkali feldspar rim), reflecting rapid changes in melt composition.

2. Quench feldspar microlites in mesostasis are alkali feldspars with compositions that overlap that of the alkalic rims of the matrix laths.
3. The pinkish clinopyroxenes are titaniferous diopsides/augites (Hole 800A) or exclusively diopsides (Site 801) with high non-quadrilateral component contents. They show little fractionation and rim zones are not markedly $\mathrm{Fe}$-rich relative to cores. One major difference was noted here in that some of the Site 801 diopsides displayed alkali pyroxene (aegirine-augite) rims separated by a sharp chemical discontinuity from the rest of the host pyroxene.

4. Brown amphibole was a rare hydrous silicate in both groups and proved to be a low-temperature, $\mathrm{Al}$-poor, winchite-ferriwinchite.

5 . Biotite was by far the most abundant hydrous phase in both alkalic groups and developed either very late in the magmatic sequence (Site 801) or during and after final consolidation (Hole 800A). The 


\section{G. ROWBOTHAM, P. A. FLOYD}

Table 8. Clinopyroxene analyses from tholeiitic basalts, Holes $801 \mathrm{C}$ and $802 \mathrm{~A}$.

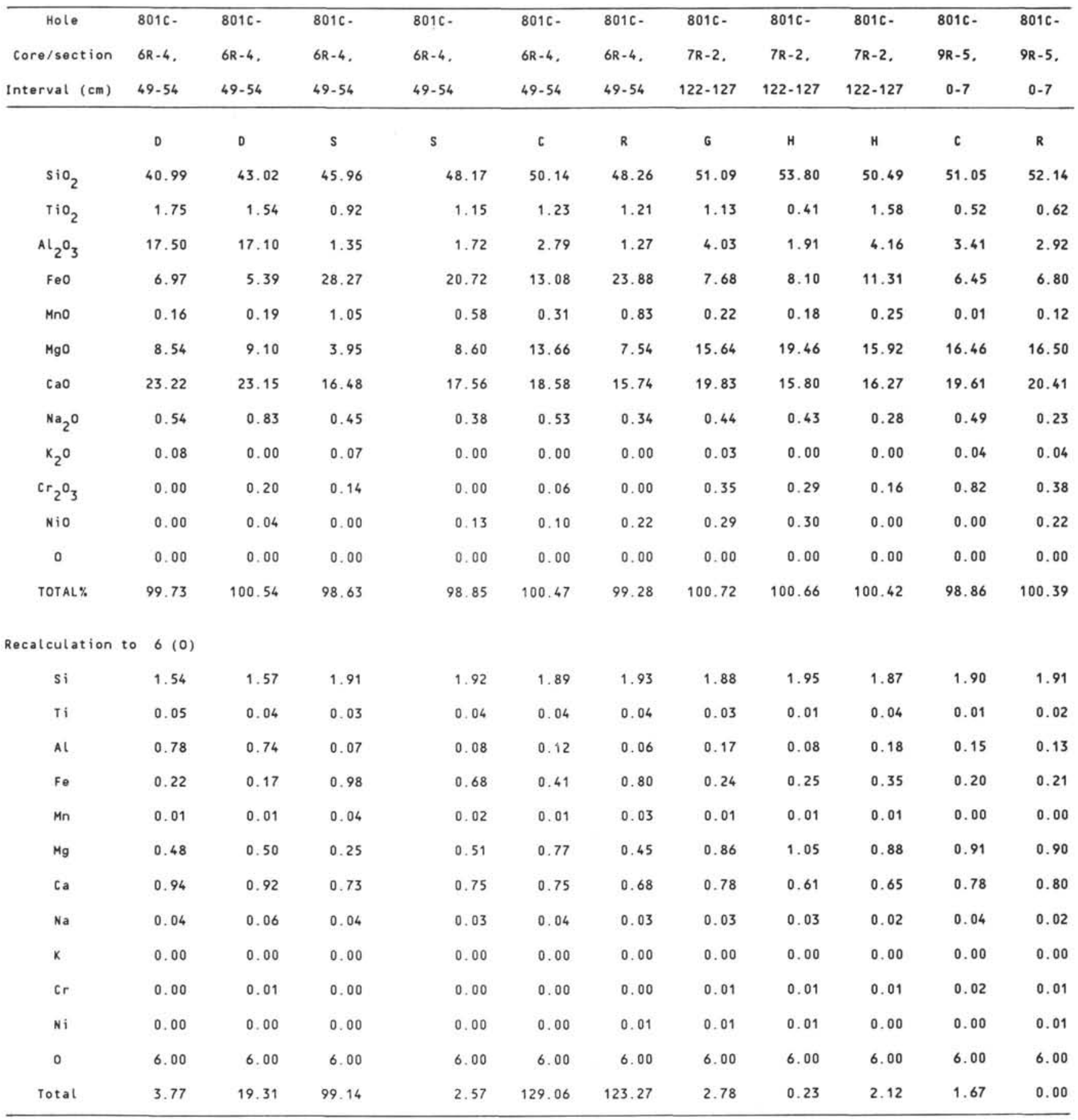

Footnote:

$\begin{array}{lll}D & = & \text { Dendritic inclusions } \\ \text { S } & = & \text { Spherulitic groundmass } \\ \text { H } & =\quad \text { Hour-glass zoned crystal }\end{array}$


Table 8 (continued).

\begin{tabular}{|c|c|c|c|c|c|c|c|c|}
\hline 802A- & 802A- & 802A- & 802A- & $802 \mathrm{~A}-$ & 802A- & 802A- & 802A- & $802 \mathrm{~A}$ - \\
\hline $58 R-3$ & $58 R-3$ & $58 R-3$ & $59 R-3$. & $59 R-3$. & $59 R-3$ & $62 R-2$ & $62 R-2$ & $62 R-2$ \\
\hline $90-95$ & $90-95$ & $90-95$ & 40.44 & $40-44$ & $40-44$ & $53-59$ & $53-59$ & $53-59$ \\
\hline R & c & c & c & c & R & c & R & R \\
\hline 53.13 & 52.13 & 52.71 & 53.10 & 53.55 & 52.69 & 52.73 & 49.73 & 50.32 \\
\hline 0.32 & 0.67 & 0.26 & 0.26 & 0.22 & 0.29 & 0.44 & 0.82 & 0.82 \\
\hline 2.08 & 6.30 & 2.54 & 2.05 & 1.88 & 1.95 & 0.91 & 2.05 & 1.65 \\
\hline 7.03 & 8.29 & 7.10 & 6.52 & 6.86 & 6.28 & 22.43 & 18.90 & 22.77 \\
\hline 0.22 & 0.23 & 0.19 & 0.27 & 0.15 & 0.34 & 0.41 & 0.45 & 0.55 \\
\hline 17.84 & 14.30 & 17.81 & 17.68 & 18.69 & 17.41 & 18.46 & 12.69 & 12.03 \\
\hline 19.14 & 16.61 & 18.56 & 19.49 & 18.42 & 19.55 & 4.78 & 14.42 & 11.45 \\
\hline 0.19 & 0.79 & 0.37 & 0.11 & 0.31 & 0.11 & 0.36 & 0.39 & 0.35 \\
\hline 0.01 & 0.08 & 0.03 & 0.03 & 0.00 & 0.03 & 0.00 & 0.07 & 0.00 \\
\hline 0.17 & 0.16 & 0.40 & 0.50 & 0.41 & 0.36 & 0.09 & 0.12 & 0.08 \\
\hline 0.12 & 0.00 & 0.05 & 0.38 & 0.32 & 0.22 & 0.28 & 0.43 & 0.00 \\
\hline 0.00 & 0.00 & 0.00 & 0.00 & 0.00 & 0.00 & 0.00 & 0.00 & 0.00 \\
\hline 100.25 & 99.54 & 100.00 & 100.37 & 100.81 & 99.21 & 100.88 & 100.05 & 100.02 \\
\hline 1.94 & 1.91 & 1.93 & 1.94 & 1.94 & 1.95 & 1.98 & 1.92 & 1.95 \\
\hline 0.01 & 0.02 & 0.01 & 0.01 & 0.01 & 0.01 & 0.01 & 0.02 & 0.02 \\
\hline 0.09 & 0.27 & 0.11 & 0.09 & 0.08 & 0.09 & 0.04 & 0.09 & 0.08 \\
\hline 0.22 & 0.25 & 0.22 & 0.20 & 0.21 & 0.19 & 0.70 & 0.61 & 0.74 \\
\hline 0.01 & 0.01 & 0.01 & 0.01 & 0.01 & 0.01 & 0.01 & 0.02 & 0.02 \\
\hline 0.97 & 0.78 & 0.97 & 0.96 & 1.01 & 0.96 & 1.03 & 0.73 & 0.69 \\
\hline 0.75 & 0.65 & 0.73 & 0.76 & 0.72 & 0.77 & 0.19 & 0.60 & 0.48 \\
\hline 0.01 & 0.06 & 0.03 & 0.01 & 0.02 & 0.01 & 0.03 & 0.03 & 0.03 \\
\hline 0.00 & 0.00 & 0.00 & 0.00 & 0.00 & 0.00 & 0.00 & 0.00 & 0.00 \\
\hline 0.01 & 0.01 & 0.01 & 0.01 & 0.01 & 0.01 & 0.00 & 0.00 & 0.00 \\
\hline 0.00 & 0.00 & 0.00 & 0.01 & 0.01 & 0.01 & 0.01 & 0.01 & 0.00 \\
\hline 6.00 & 6.00 & 6.00 & 6.00 & 6.00 & 6.00 & 6.00 & 6.00 & 6.00 \\
\hline 800.88 & 15.51 & 4.01 & 4.01 & 4.02 & 4.00 & 4.00 & 4.03 & 4.00 \\
\hline
\end{tabular}

late-magmatic biotites were distinctive in having higher $\mathrm{Ti}$ contents and $\mathrm{Mg} / \mathrm{Fe}$ ratios, indicative of higher temperatures of formation.

In both alkalic groups, minor olivine and plagioclase phenocrysts were the first phases to crystallize, although most units are aphyric in character. A broadly similar petrogenetic scheme can be considered common to both sills and lava flow interiors. The strongly zoned matrix feldspar has cores of similar composition to plagioclase phenocrysts and crystallized under nonequilibrium conditions that gradually became more sodic. Elongate prisms of titaniferous diopside crystallized at about the same time or slightly later, but did not undergo major internal fractionation (uniform $\mathrm{Fe} / \mathrm{Mg}$ ratios) due to the precipitation of abundant titanomagnetite that reduced the Fe and Ti content of the remaining melt. As anhydrous crystallization proceeded, the water content of the melt built up and $\mathrm{fO}_{2}$ increased, allowing the growth of minor brown amphibole ( with high $\mathrm{Fe}^{3+}$ ). Any melt remaining was now $\mathrm{K}$-rich and produced alkalic rims to the plagioclases and precipitated magmatic biotite that nucleated on the opaques. Na-rich fluids also developed alkali pyroxene rims to the diopsides in Site 801 basalt flow interiors. The final melt was quenched as glassy mesostasis within which alkali feldspar microlites rapidly grew. 
G. ROWBOTHAM, P. A. FLOYD

Table 9. Olivine analyses from tholeiitic basalts, Holes $801 \mathrm{C}$ and $802 \mathrm{~A}$.

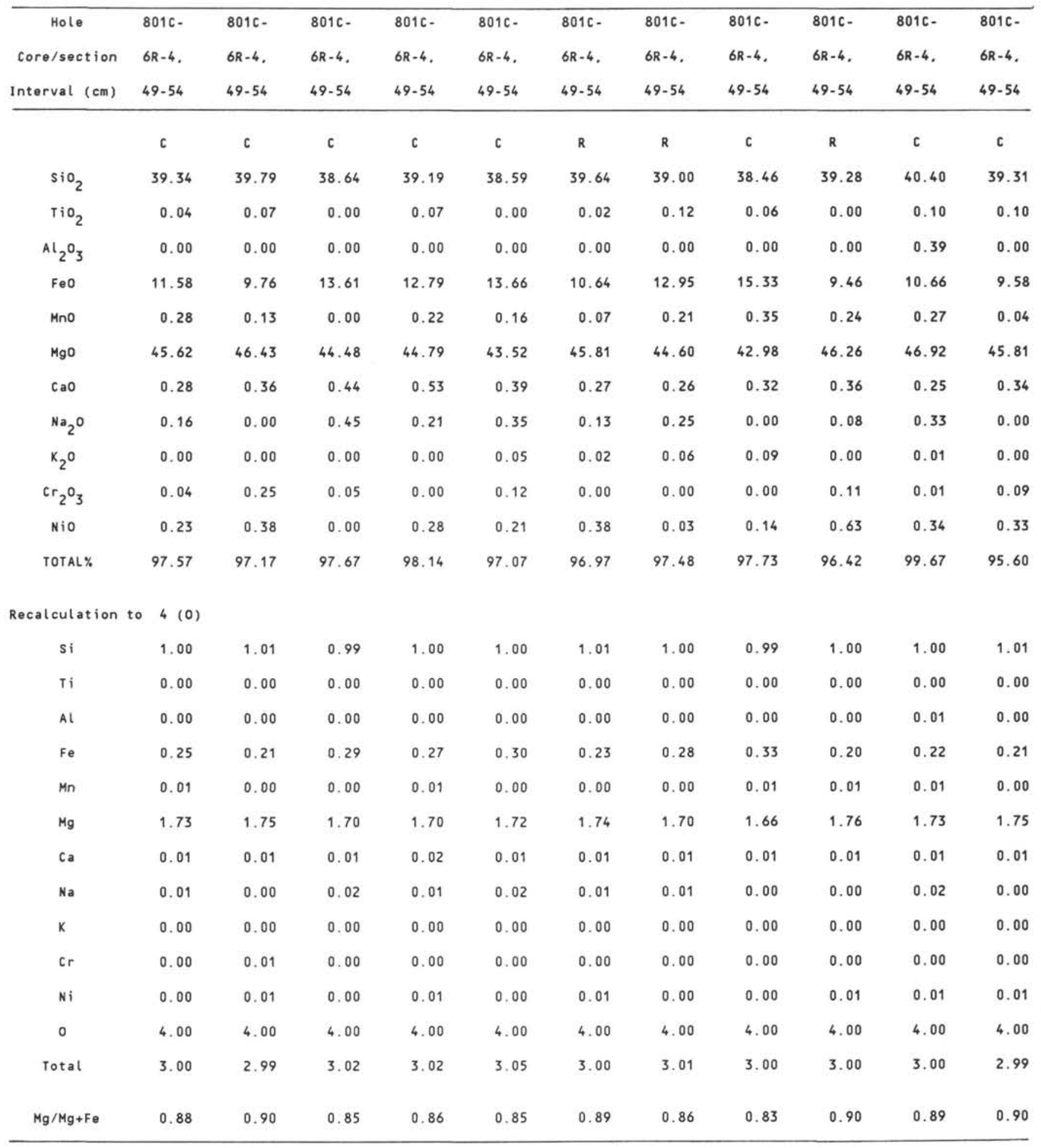

Footnote:

c $=$ Core

$R=R i m$ 
Table 9 (continued).

\begin{tabular}{|c|c|c|c|c|c|c|c|}
\hline $801 \mathrm{C}$ - & 802A- & 802A- & 802A- & $802 \mathrm{~A}-$ & $802 \mathrm{~A}-$ & 802A- & 802A- \\
\hline $6 R-4$. & $58 R-2$. & $58 R-2$, & $58 R-2$. & $58 R-2$. & $58 R-2$ & $58 R-2$. & $58 R-2$, \\
\hline $49-54$ & $99-102$ & $99-102$ & $99-102$ & $99-102$ & $99-102$ & $99-102$ & $99-102$ \\
\hline R & c & c & R & c & R & c & c \\
\hline 39.02 & 39.93 & 39.90 & 39.92 & 39.64 & 39.78 & 39.63 & 39.52 \\
\hline 0.00 & 0.00 & 0.00 & 0.00 & 0.00 & 0.00 & 0.05 & 0.08 \\
\hline 0.10 & 0.26 & 0.39 & 0.25 & 0.28 & 0.29 & 0.32 & 0.15 \\
\hline 14.19 & 18.12 & 19.13 & 18.46 & 18.38 & 18.71 & 18.79 & 18.10 \\
\hline 0.28 & 0.38 & 0.25 & 0.06 & 0.34 & 0.26 & 0.28 & 0.23 \\
\hline 44.01 & 42.14 & 42.25 & 42.03 & 42.04 & 42.33 & 42.48 & 41.35 \\
\hline 0.39 & 0.32 & 0.36 & 0.29 & 0.35 & 0.33 & 0.36 & 0.36 \\
\hline 0.20 & 0.16 & 0.14 & 0.36 & 0.17 & 0.24 & 0.43 & 0.00 \\
\hline 0.00 & 0.02 & 0.02 & 0.02 & 0.00 & 0.01 & 0.00 & 0.03 \\
\hline 0.10 & 0.00 & 0.03 & 0.00 & 0.00 & 0.07 & 0.00 & 0.13 \\
\hline 0.17 & 0.68 & 0.47 & 0.83 & 0.40 & 1.04 & 0.60 & 0.41 \\
\hline 98.52 & 101.99 & 102.92 & 102.22 & 101.59 & 103.05 & 102.94 & 100.35 \\
\hline 1.00 & 1.00 & 0.99 & 1.00 & 1.00 & 0.99 & 0.99 & 1.01 \\
\hline 0.00 & 0.00 & 0.00 & 0.00 & 0.00 & 0.00 & 0.00 & 0.00 \\
\hline 0.00 & 0.01 & 0.01 & 0.01 & 0.01 & 0.01 & 0.01 & 0.00 \\
\hline 0.30 & 0.38 & 0.40 & 0.39 & 0.39 & 0.39 & 0.39 & 0.39 \\
\hline 0.01 & 0.01 & 0.01 & 0.00 & 0.01 & 0.01 & 0.01 & 0.01 \\
\hline 1.67 & 1.57 & 1.57 & 1.57 & 1.58 & 1.57 & 1.58 & 1.57 \\
\hline 0.01 & 0.01 & 0.01 & 0.01 & 0.01 & 0.01 & 0.01 & 0.01 \\
\hline 0.01 & 0.01 & 0.01 & 0.02 & 0.01 & 0.01 & 0.02 & 0.00 \\
\hline 0.00 & 0.00 & 0.00 & 0.00 & 0.00 & 0.00 & 0.00 & 0.00 \\
\hline 0.00 & 0.00 & 0.00 & 0.00 & 0.00 & 0.00 & 0.00 & 0.00 \\
\hline 0.00 & 0.01 & 0.01 & 0.02 & 0.01 & 0.02 & 0.01 & 0.01 \\
\hline 4.00 & 4.00 & 4.00 & 4.00 & 4.00 & 4.00 & 4.00 & 4.00 \\
\hline 3.01 & 3.00 & 3.00 & 3.01 & 3.00 & 3.01 & 3.02 & 2.99 \\
\hline 0.85 & 0.81 & 0.80 & 0.80 & 0.80 & 0.80 & 0.80 & 0.80 \\
\hline
\end{tabular}

\section{Tholeiitic Basalts}

The relatively uniform chemical composition of the pillow lavas from Hole $802 \mathrm{~A}$ is reflected in the major silicate phases. Phenocrystic olivine $\left(\mathrm{Fo}_{80}\right)$ and low- $\mathrm{Ti}$ augite show no variation in $\mathrm{Fe} / \mathrm{Mg}$ ratios, although minor components can be highly variable (for example, $\mathrm{Cr}$, $\mathrm{Ti}$, and $\mathrm{Al}$ in clinopyroxene). Plagioclase megaphenocrysts do show some minor zoning $\left(\mathrm{An}_{86}\right.$ to $\left.\mathrm{An}_{50}\right)$, although there is general compositional overlap between microphenocrysts and quenched laths in the matrix.
More varied chemistry is exhibited by the major phases in Site 801 lavas, especially between mineral species that crystallized at different stages in the early glomerophyric groups, as microphenocrysts, and finally as quenched variolites. The primitive group of tholeiitic lavas is characterized by extreme compositions of the main phenocrystic phases: chrome spinels $(\mathrm{Cr} / \mathrm{Cr}+\mathrm{Al}=0.2-0.4)$, very Ni-rich olivines $\left(\mathrm{Fo}_{90}\right)$, and highly calcic plagioclase $\left(\mathrm{An}_{90}\right)$. The compositional range of the phenocrysts is typical of some types of MORB and, in particular, characteristic of very primitive melt compositions. Clinopyroxene is a minor 

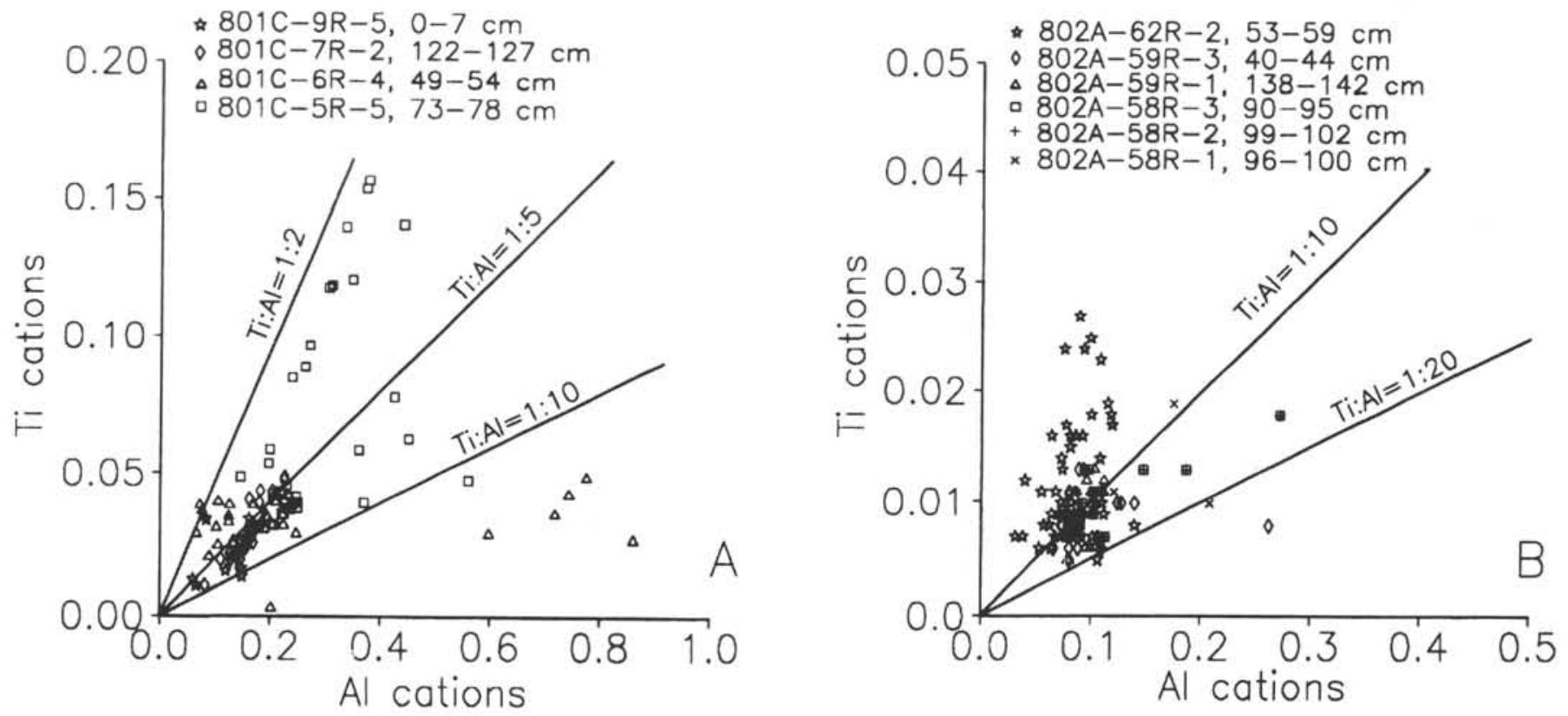

Figure 10. Minor components, $\mathrm{Ti}$ and $\mathrm{Al}$, in clinopyroxenes from tholeiitic basalt flows. A. Hole 80IC. B. Hole 802A.

phase in these basalts, but is present in the chemically evolved group as zoned diopside-augite microphenocrysts. Spinel occurs both as micro-phenocrysts (with high $\mathrm{Cr}$ ) and also as inclusions within olivine and plagioclase which display chemical variation depending on the precipitation of other major silicates. Compositional variation relative to crystallization history is exhibited by plagioclase: individual megacrysts and those in glomerophyric clumps are the most calcic $\left(\mathrm{An}_{90}\right.$; some zoned to $\mathrm{An}_{50}$ ) and microphenocrysts are marginally more sodic $\left(A n_{75-80}\right)$, whereas plagioclase microlites and serrated laths in variolites show the greatest variation $\left(\mathrm{An}_{65-24}\right)$.

Crystallization history for the primitive tholeiites starts with the growth of euhedral $\mathrm{Cr}$-rich spinel microphenocrysts, closely followed by olivine and then calcic plagioclase. However, during olivine precipitation further spinel growth occurred but with a lower $\mathrm{Cr}$ content (possibly due to an increase in $\mathrm{fO}_{2}$ ). Renewed spinel growth also occurred during plagioclase precipitation when competition for $\mathrm{Al}$ once again increased spinel $\mathrm{Cr} / \mathrm{Cr}+\mathrm{Al}$ ratios. A possible earlier phase of high-pressure

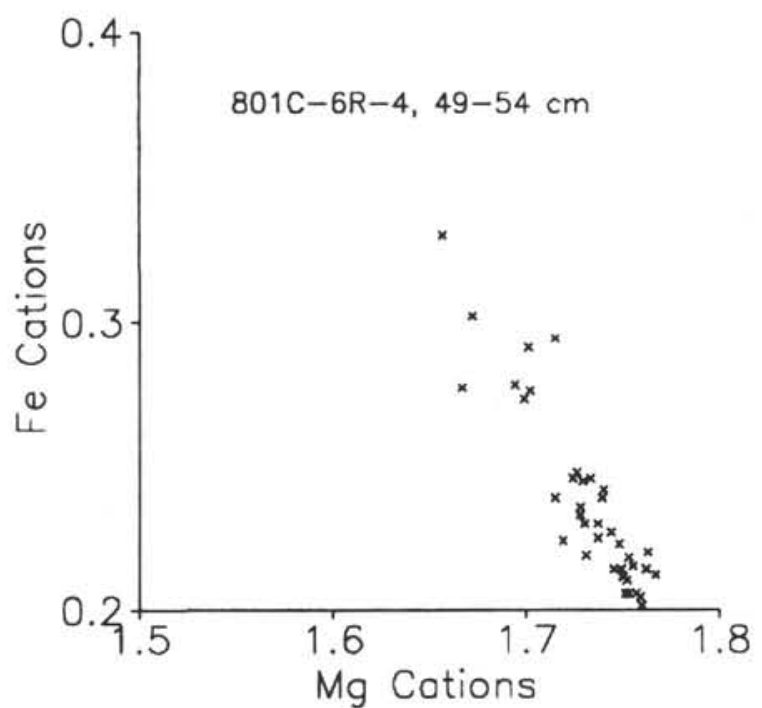

Figure 11. Compositional variation in olivines from Sample 129-801C-6R-4, $49-54 \mathrm{~cm}$, in terms of $\mathrm{Mg}$ and $\mathrm{Fe}$ components. crystallization may be recorded by high-Al pyroxene (fassaite)-bearing spheroids enclosed in the olivine microphenocrysts. After precipitation of the major primitive phenocryst compositions (in a magma chamber) further microphenocrystic growth in glomerophyric clumps may have taken place nearer the seafloor during upward transport. Most microphenocrysts of plagioclase and clinopyroxene are strongly zoned and indicative of rapidly changing melt conditions. Quenching of the remaining melt on extrusion produced clinopyroxene and plagioclase variolites with the most evolved chemical compositions.

It has been suggested that chemical subgroups within the evolved tholeiitic group of Hole 801 are a consequence of magma mixing between fractionated compositions and a series of more primitive melt inputs (Floyd and Castillo, this volume). The evolved tholeiites are generally aphyric or poorly olivine and plagioclase-clinopyroxene glomerophyric and apparently do not contain resorbed and strongly zoned megaphenocrysts (for example, plagioclase) of primitive composition similar to those in the primitive group tholeiites. Also, phenocryst phases have not been analyzed in detail to confirm if they have core compositions more primitive than expected for their host melt composition, although some matrix plagioclases in flow interiors are zoned with bytownite cores. At present, magma mixing is based largely on bulk rock-chemical features, rather than strong petrographic evidence and phase analysis.

Table 10. Glass analyses from tholeiitic basalt Sample 129-802A58R-2, 99-102 cm.

\begin{tabular}{lrrrrrr}
\hline \multicolumn{1}{c}{ Analysis } & \multicolumn{1}{c}{1} & \multicolumn{1}{c}{2} & \multicolumn{1}{c}{3} & \multicolumn{1}{c}{4} & \multicolumn{1}{c}{5} & 6 \\
\hline $\mathrm{SiO}_{2}$ & 50.88 & 51.39 & 51.77 & 51.62 & 51.65 & 51.62 \\
$\mathrm{TiO}_{2}$ & 1.16 & 1.17 & 1.22 & 1.21 & 1.22 & 1.19 \\
$\mathrm{Al}_{2} \mathrm{O}_{3}$ & 13.29 & 13.37 & 13.40 & 13.66 & 13.55 & 13.48 \\
$\mathrm{FeO}$ & 11.83 & 11.55 & 11.80 & 11.37 & 11.70 & 11.89 \\
$\mathrm{MnO}$ & 0.25 & 0.13 & 0.21 & 0.23 & 0.32 & 0.13 \\
$\mathrm{MgO}$ & 6.89 & 6.75 & 6.92 & 6.92 & 6.78 & 6.98 \\
$\mathrm{CaO}$ & 11.83 & 12.15 & 11.26 & 11.45 & 11.38 & 11.37 \\
$\mathrm{Na}$ & 2.24 & 2.21 & 2.20 & 2.35 & 2.23 & 2.16 \\
$\mathrm{~K}_{2} \mathrm{O}$ & 0.16 & 0.13 & 0.14 & 0.16 & 0.13 & 0.17 \\
$\mathrm{Total}$ & 98.53 & 98.85 & 98.92 & 98.97 & 98.96 & 98.99 \\
$\mathrm{MgO} / \mathrm{MgO}+\mathrm{FeO}$ & 0.37 & 0.37 & 0.37 & 0.38 & 0.37 & 0.37 \\
\hline
\end{tabular}



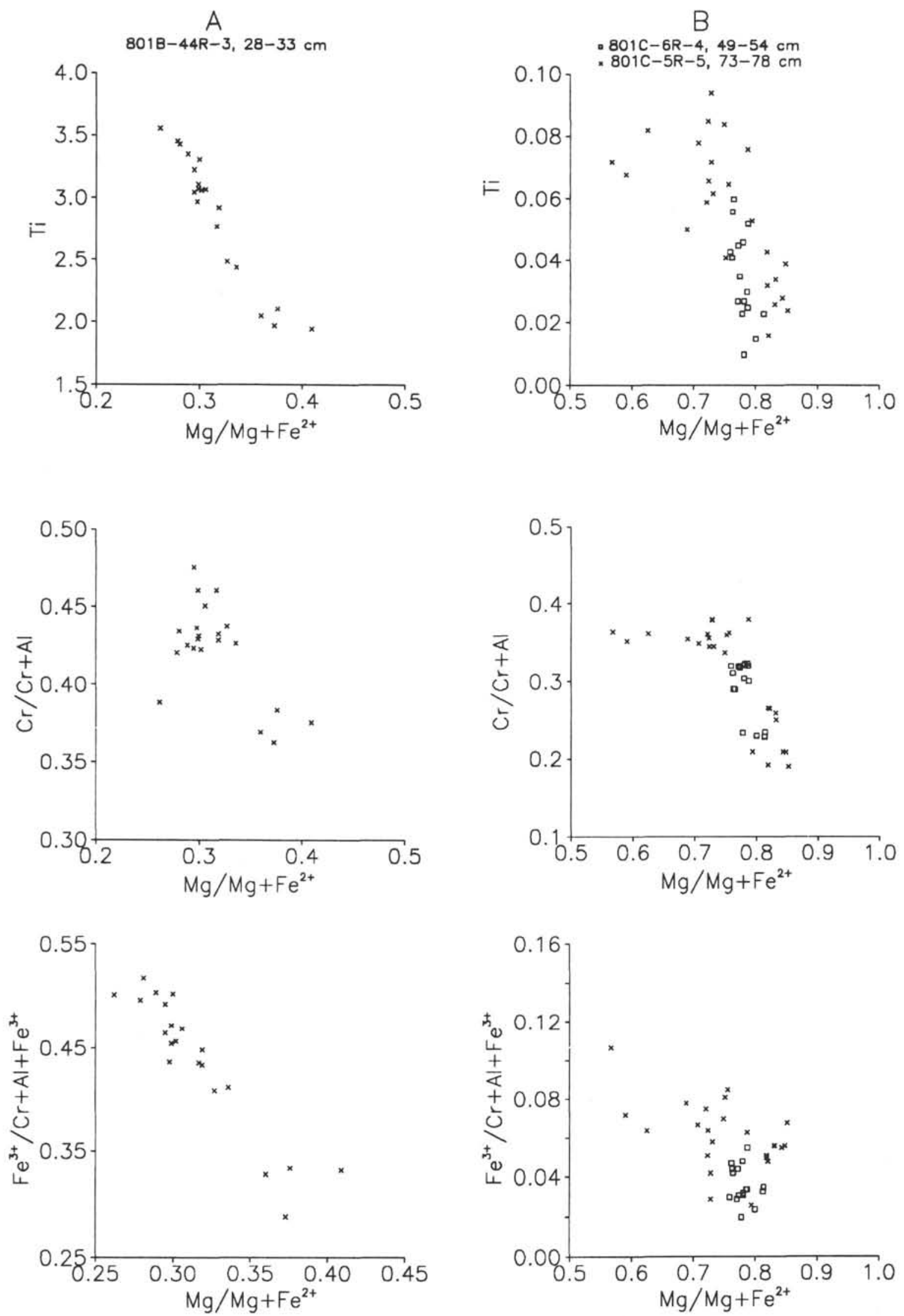

Figure 12. Composition of spinels from Holes $801 \mathrm{~B}$ and $801 \mathrm{C}$ in terms of $\mathrm{Ti}$ content, and $\mathrm{Cr} /(\mathrm{Cr}+\mathrm{Al})$ and $\mathrm{Fe}^{3+} /\left(\mathrm{Fe}^{3+}+\mathrm{Cr}+\mathrm{Al}\right)$ ratios. A. Titaniferous spinels from alkalic intrusive, Sample 129-801B-44R-3, 28-33 cm. B. Chrome spinels from primitive tholeiites, Samples $129-801 \mathrm{C}-5 \mathrm{R}-5,73-78 \mathrm{~cm}$ and $801 \mathrm{C}-6 \mathrm{R}-4,40-54 \mathrm{~cm}$. 
Table 11. Spinel analyses from tholeiitic basalts, Holes 801B and 801C.

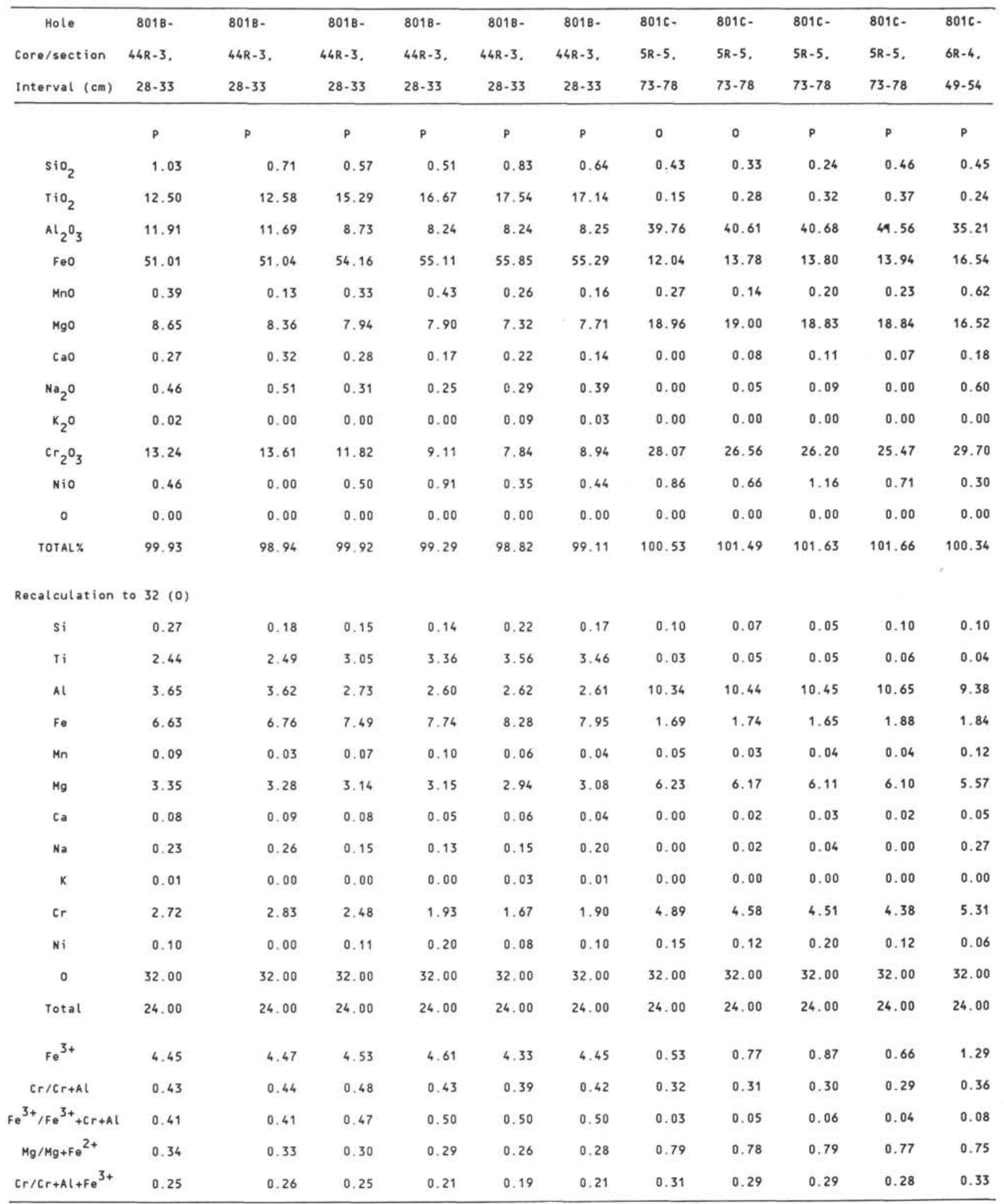

\section{Footnote :}

$P=$ Inclusion in plagioclase

$M=$ Microphenocryst

$0=$ Inclusion in olivine 
Table 11 (continued).

\begin{tabular}{|c|c|c|c|c|c|c|c|c|}
\hline $801 C$ - & $801 \mathrm{C}-$ & $801 \mathrm{C}-$ & $801 \mathrm{C}$ - & $801 C$ - & 8016 - & $801 \mathrm{C}$ - & $801 \mathrm{C}-$ & $801 \mathrm{C}$ - \\
\hline $6 R-4$. & $6 R-4$ & $6 R-4$. & $6 R-4$. & $6 R-4$ & $6 R-4$. & $6 R-4$ & $6 R-4$ & $6 R-4$. \\
\hline 49.54 & $49-54$ & $49-54$ & $49-54$ & $49-54$ & $49-54$ & $49-54$ & $49-54$ & 49.54 \\
\hline$P$ & 0 & M & $M$ & 0 & 0 & 0 & 0 & 0 \\
\hline 0.34 & 0.15 & 0.21 & 0.61 & 0.21 & 0.00 & 0.46 & 0.51 & 0.17 \\
\hline 0.38 & 0.16 & 0.42 & 0.50 & 0.32 & 0.17 & 0.21 & 0.20 & 0.09 \\
\hline 34.73 & 44.35 & 34.85 & 36.58 & 48.38 & 47.44 & 45.24 & 44.17 & 43.64 \\
\hline 16.85 & 12.00 & 14.81 & 15.96 & 11.46 & 11.43 & 12.24 & 12.33 & 11.79 \\
\hline 0.26 & 0.10 & 0.22 & 0.00 & 0.09 & 0.24 & 0.17 & 0.00 & 0.15 \\
\hline 16.63 & 19.40 & 16.97 & 17.14 & 19.87 & 19.67 & 19.97 & 19.67 & 19.32 \\
\hline 0.30 & 0.03 & 0.00 & 0.04 & 0.05 & 0.00 & 0.05 & 0.00 & 0.06 \\
\hline 0.50 & 0.51 & 0.02 & 0.40 & 0.05 & 0.45 & 0.51 & 0.54 & 0.33 \\
\hline 0.00 & 0.07 & 0.08 & 0.00 & 0.00 & 0.06 & 0.09 & 0.06 & 0.00 \\
\hline 29.59 & 23.28 & 31.84 & 30.30 & 19.16 & 18.80 & 22.75 & 23.99 & 23.60 \\
\hline 0.31 & 0.47 & 0.43 & 0.04 & 0.38 & 0.38 & 0.24 & 0.17 & 0.53 \\
\hline 0.00 & 0.00 & 0.00 & 0.00 & 0.00 & 0.00 & 0.00 & 0.00 & 0.00 \\
\hline 99.89 & 100.49 & 99.85 & 101.55 & 99.96 & 98.64 & 101.93 & 101.63 & 99.68 \\
\hline 0,08 & 0.03 & 0.05 & 0.14 & 0.05 & 0.00 & 0.10 & 0.11 & 0.04 \\
\hline 0.07 & 0.03 & 0.07 & 0.09 & 0.05 & 0.03 & 0.03 & 0.03 & 0.02 \\
\hline 9.28 & 11.26 & 9.38 & 9.60 & 12.18 & 12.06 & 11.30 & 11.10 & 11.20 \\
\hline 1.83 & 1.26 & 2.16 & 2.18 & 1.64 & 1.18 & 1.28 & 1.38 & 1.37 \\
\hline 0.05 & 0.02 & 0.04 & 0.00 & 0.02 & 0.04 & 0.03 & 0.00 & 0.03 \\
\hline 5.69 & 6.23 & 5.77 & 5.69 & 6.33 & 6.33 & 6.31 & 6.25 & 6.27 \\
\hline 0.07 & 0.01 & 0.01 & 0.01 & 0.01 & 0.00 & 0.01 & 0.00 & 0.01 \\
\hline 0.22 & 0.21 & 0.01 & 0.17 & 0,02 & 0.02 & 0.21 & 0.22 & 0.14 \\
\hline 0.00 & 0.02 & 0.02 & 0.00 & 0.00 & 0.02 & 0.02 & 0.02 & 0.00 \\
\hline 5.30 & 3.96 & 5.75 & 5.34 & 3.24 & 3.21 & 3.78 & 4.04 & 4.06 \\
\hline 0.06 & 0.08 & 0.08 & 0.01 & 0.07 & 0.07 & 0.04 & 0.03 & 0.09 \\
\hline 32.00 & 32.00 & 32.00 & 32.00 & 32.00 & 32.00 & 32.00 & 32.00 & 32.00 \\
\hline 24.00 & 24.00 & 24.00 & 24.00 & 24.00 & 24.00 & 24.00 & 24.00 & 24.00 \\
\hline 1.36 & 0.90 & 0.67 & 0.80 & 0.41 & 0.88 & 0.89 & 0.82 & 0.78 \\
\hline 0.36 & 0.26 & 0.38 & 0.36 & 0.21 & 0.21 & 0.25 & 0.27 & 0.27 \\
\hline 0.09 & 0.06 & 0.04 & 0.05 & 0.03 & 0.06 & 0.06 & 0.05 & 0.05 \\
\hline 0.76 & 0.83 & 0.73 & 0.72 & 0.79 & 0.84 & 0.83 & 0.82 & 0.82 \\
\hline 0.33 & 0.25 & 0.36 & 0.34 & 0.20 & 0.20 & 0.24 & 0.25 & 0.25 \\
\hline
\end{tabular}

\section{Secondary Phases and Alteration}

Phyllosilicates dominate secondary assemblages in both the alkalic and tholeiitic basalts and include various colored smectites ( $\mathrm{Fe}$-, $\mathrm{Mg}$-, and $\mathrm{Al}$-saponites), chlorite-smectite, and celadonite. Smectite compositions commonly reflect the nature of the replaced host, such that greenish $\mathrm{Mg}$-saponites replace primary mafic minerals, although olivine can also be pseudomorphed by either $\mathrm{Al}$-rich saponites with high $\mathrm{Fe} / \mathrm{Mg}$ ratios or $\mathrm{Al}$-rich chlorite-smectite. Interstitial areas, especially glassy mesostasis, and pillow rims are replaced by various brownish $\mathrm{Fe}$-saponites with variable $\mathrm{Fe} / \mathrm{Mg}$ ratios (and proportions of $\mathrm{Al}^{\mathrm{iv}}$ ), that may reflect the composition of early quenched (parental) 
G. ROWBOTHAM, P. A. FLOYD

Table 12. Smectite analyses from alkalic dolerites and basalts, Holes 800A, 801B, and 801C.

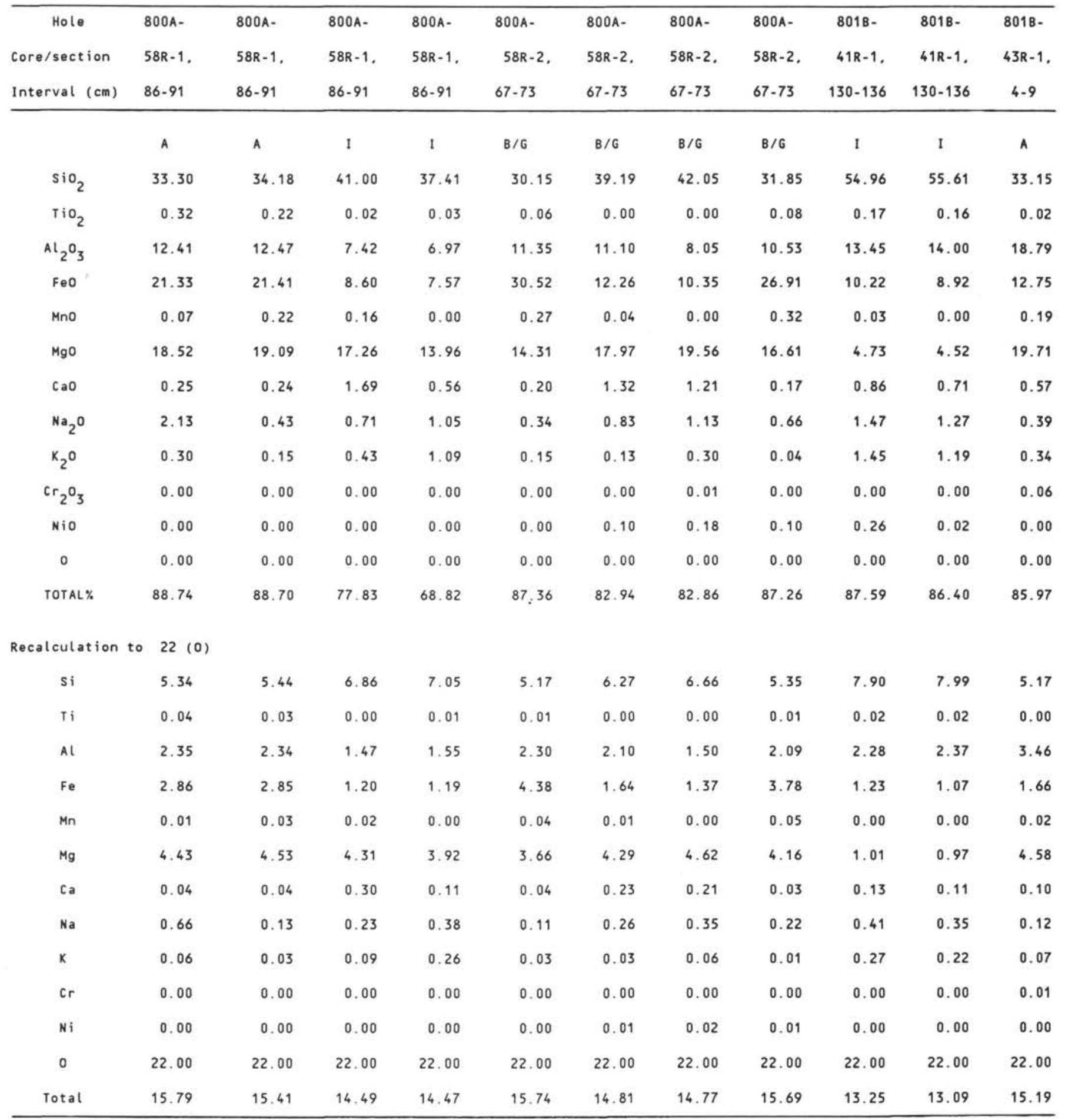

Footnote:

$\begin{array}{rlll}A & = & \text { Replacement of biotite } \\ B / G & = & \text { Bright green matrix replacement } \\ \text { I } & = & \text { Interstitial smectite } \\ 0 & = & \text { Replacement of olivine } \\ M & = & \text { Matrix smectite }\end{array}$


Table 12 (continued).

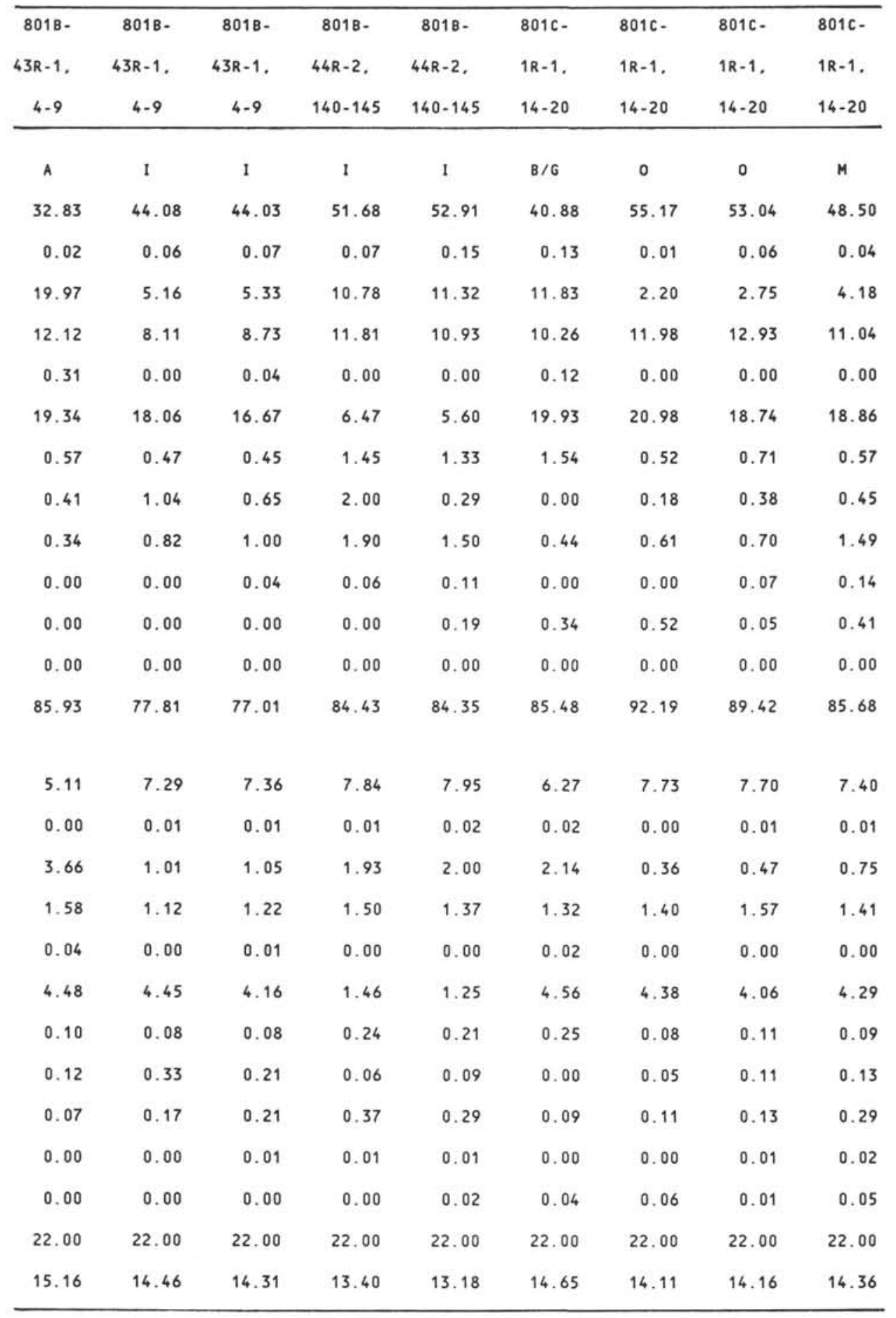

melts vs. late interstitial, Fe-rich melts. Very bright green clays replacing late magmatic biotite in the alkalic rocks are $\mathrm{Al}$ - and $\mathrm{Fe}$-rich chlorite-smectites. Celadonite may also be associated with altered interstitial areas and generally has higher and more varied $\mathrm{Fe} / \mathrm{Mg}$ ratios than the associated smectites.

Some carbonates infilling veins may show a zonation from finegrained granular ferroan dolomites to coarse rhombic calcite in the centers.

\section{ACKNOWLEDGMENTS}

The analyses were performed in the electron microprobe facility in the Geology Department, University of Manchester, United Kingdom, and we are grateful to Professor C. D. Curtis for permission to use the instruments. We thank Tim Hopkins, Dave Plant, and Dave Wright for keeping the microprobes in such good working order. Mike 


\section{G. ROWBOTHAM, P. A. FLOYD}

Table 13. Smectite analyses from tholeiitic basalts, Holes 801C and 802A.

\begin{tabular}{|c|c|c|c|c|c|c|c|c|c|c|c|}
\hline $\begin{array}{l}\text { Hole } \\
\text { Core/section } \\
\text { Interval }(\mathrm{cm})\end{array}$ & $\begin{array}{l}801 C- \\
5 R-5 . \\
73-78\end{array}$ & $\begin{array}{l}801 C- \\
5 R-5 \\
73-78\end{array}$ & $\begin{array}{l}801 C- \\
5 R-5 \\
73-78\end{array}$ & $\begin{array}{l}801 C- \\
6 R-4 . \\
49-54\end{array}$ & $\begin{array}{l}801 C- \\
6 R-4 . \\
49-54\end{array}$ & $\begin{array}{l}801 C- \\
6 R-4 . \\
49-54\end{array}$ & $\begin{array}{l}801 C- \\
6 R-4 . \\
49-54\end{array}$ & $\begin{array}{c}801 C- \\
7 R-2 \\
122-127\end{array}$ & $\begin{array}{c}801 C- \\
7 R-2 \\
122-127\end{array}$ & $\begin{array}{c}801 C- \\
7 R-2 \\
122-127\end{array}$ & $\begin{array}{l}801 C- \\
9 R-5 . \\
0-7\end{array}$ \\
\hline & P & P & 0 & H & M & $v$ & v & B & $Y / B$ & B & B \\
\hline $\mathrm{SiO}_{2}$ & 43.32 & 43.19 & 50.80 & 26.16 & 25.86 & 36.96 & 33.20 & 53.03 & 51.41 & 54.78 & 50.52 \\
\hline $\mathrm{TiO}_{2}$ & 0.06 & 0.05 & 0.02 & 0.43 & 0.41 & 0.00 & 0.00 & 0.29 & 0.07 & 0.54 & 0.30 \\
\hline $\mathrm{Al}_{2} \mathrm{O}_{3}$ & 6.41 & 6.52 & 1.32 & 4.69 & 4.05 & 3.48 & 2.68 & 6.28 & 4.62 & 8.49 & 2.92 \\
\hline $\mathrm{FeO}$ & 8.70 & 9.33 & 14.64 & 26.65 & 31.25 & 21.93 & 23.20 & 16.88 & 16.47 & 14.64 & 18.38 \\
\hline Mno & 0.00 & 0.07 & 0.13 & 0.16 & 0.07 & 0.18 & 0.38 & 0.01 & 0.00 & 0.03 & 0.08 \\
\hline $\mathrm{MgO}$ & 18.73 & 18.61 & 16.23 & 12.99 & 9.71 & 16.08 & 15.37 & 12.78 & 7.85 & 11.75 & 16.35 \\
\hline CaO & 1.03 & 1.09 & 0.66 & 1.09 & 2.06 & 0.82 & 0.70 & 1.65 & 1.39 & 1.92 & 0.89 \\
\hline $\mathrm{Na}_{2} \mathrm{O}$ & 0.47 & 0.49 & 0.35 & 0.10 & 1.41 & 0.48 & 0.40 & 0.56 & 0.64 & 0.36 & 0.64 \\
\hline $\mathrm{K}_{2} \mathrm{O}$ & 1.01 & 0.89 & 0.74 & 0.89 & 0.61 & 1.05 & 1.12 & 0.55 & 0.59 & 0.78 & 0.54 \\
\hline $\mathrm{Cr}_{2} \mathrm{O}_{3}$ & 0.05 & 0.00 & 0.05 & 0.00 & 0.00 & 0.00 & 0.00 & 0.07 & 0.03 & 0.00 & 0.00 \\
\hline Nio & 0.23 & 0.00 & 0.36 & 0.00 & 0.00 & 0.00 & 0.00 & 0.20 & 0.20 & 0.35 & 0.13 \\
\hline 0 & 0.00 & 0.00 & 0.00 & 0.00 & 0.00 & 0.00 & 0.00 & 0.00 & 0.00 & 0.00 & 0.00 \\
\hline TOTAL: & 80.01 & 80.24 & 85.32 & 73.15 & 75.43 & 80.98 & 77.04 & 92.29 & 83.25 & 93.64 & 90.75 \\
\hline
\end{tabular}

Recalculation to $22(0)$

\begin{tabular}{cccccccccccc} 
Si & 7.02 & 7.00 & 7.85 & 5.49 & 5.46 & 6.53 & 6.14 & 7.59 & 8.12 & 7.61 & 7.46 \\
$\mathrm{Ti}$ & 0.01 & 0.01 & 0.00 & 0.07 & 0.07 & 0.00 & 0.00 & 0.03 & 0.01 & 0.06 & 0.03 \\
$\mathrm{AL}$ & 1.22 & 1.24 & 0.24 & 1.16 & 1.01 & 0.72 & 0.60 & 1.06 & 0.86 & 1.39 & 0.51 \\
$\mathrm{Fe}$ & 1.18 & 1.26 & 1.89 & 4.67 & 5.52 & 3.24 & 3.69 & 2.02 & 2.18 & 1.70 & 2.28 \\
$\mathrm{Hn}$ & 0.00 & 0.01 & 0.02 & 0.03 & 0.01 & 0.03 & 0.06 & 0.00 & 0.00 & 0.00 & 0.01 \\
$\mathrm{Mg}$ & 4.53 & 4.49 & 3.74 & 4.06 & 3.06 & 4.23 & 4.36 & 2.73 & 1.85 & 2.43 & 3.61 \\
$\mathrm{Ca}$ & 0.18 & 0.19 & 0.11 & 0.24 & 0.47 & 0.16 & 0.14 & 0.25 & 0.24 & 0.29 & 0.14 \\
$\mathrm{Na}$ & 0.15 & 0.15 & 0.11 & 0.04 & 0.58 & 0.16 & 0.15 & 0.16 & 0.20 & 0.10 & 0.18 \\
$\mathrm{~K}$ & 0.21 & 0.18 & 0.15 & 0.24 & 0.17 & 0.24 & 0.27 & 0.10 & 0.12 & 0.14 & 0.10 \\
$\mathrm{Cr}$ & 0.01 & 0.00 & 0.01 & 0.00 & 0.00 & 0.00 & 0.00 & 0.01 & 0.00 & 0.00 & 0.00 \\
$\mathrm{Ni}$ & 0.03 & 0.00 & 0.05 & 0.00 & 0.00 & 0.00 & 0.00 & 0.02 & 0.03 & 0.04 & 0.02 \\
$\mathrm{O}$ & 22.00 & 22.00 & 22.00 & 22.00 & 22.00 & 22.00 & 22.00 & 22.00 & 22.00 & 22.00 & 22.00 \\
Total & 14.53 & 14.54 & 14.15 & 16.00 & 16.34 & 15.31 & 15.41 & 13.97 & 13.59 & 13.76 & 14.33 \\
\hline
\end{tabular}

\footnotetext{
$P=$ Plates of smectite

$0=$ Replacement of olivine

$M=$ Matrix smectite

$v=$ Smectite in vescicle

$B=$ Brown matrix smectite

$Y / B=$ Yellow-brown matrix smectite
} 
Table 13 (continued).

\begin{tabular}{lrrrrrrrr}
\hline $801 C-$ & $802 A-$ & $802 A-$ & $802 A-$ & $802 A-$ & $802 A-$ & $802 A-$ & $802 A-$ & $802 A-$ \\
$9 R-5$. & $58 R-2$. & $58 R-2$. & $58 R-2$. & $58 R-2$. & $59 R-1$. & $59 R-1$. & $59 R-3$. & $59 R-3$. \\
$0-7$ & $99-102$ & $99-102$ & $99-102$ & $99-102$ & $136-142$ & $136-142$ & $40-44$ & $40-44$ \\
\hline
\end{tabular}

$\begin{array}{lllllllll}\text { B } & 0 & 0 & 0 & 0 & 70 & 70 & \text { H } & \text { M } \\ 50.37 & 46.50 & 46.49 & 47.74 & 48.25 & 53.60 & 50.32 & 45.31 & 50.11 \\ 0.33 & 0.55 & 0.46 & 0.08 & 0.14 & 0.49 & 0.21 & 0.05 & 0.26 \\ 2.81 & 7.56 & 7.41 & 5.94 & 5.78 & 6.61 & 4.37 & 5.77 & 4.48 \\ 17.95 & 19.82 & 20.03 & 17.46 & 17.13 & 11.82 & 13.12 & 14.26 & 13.48 \\ 0.05 & 0.02 & 0.00 & 0.03 & 0.00 & 0.00 & 0.07 & 0.00 & 0.00 \\ 16.15 & 12.81 & 12.32 & 15.68 & 15.86 & 15.69 & 18.24 & 14.54 & 18.13 \\ 0.68 & 2.32 & 2.31 & 1.67 & 1.66 & 1.71 & 1.67 & 1.78 & 1.82 \\ 0.55 & 0.41 & 0.53 & 0.21 & 0.16 & 0.29 & 0.30 & 0.62 & 0.29 \\ 0.56 & 1.02 & 1.05 & 0.87 & 0.98 & 0.95 & 0.55 & 0.86 & 0.53 \\ 0.12 & 0.01 & 0.13 & 0.01 & 0.00 & 0.06 & 0.06 & 0.01 & 0.01 \\ 0.09 & 0.25 & 0.07 & 0.08 & 0.17 & 0.33 & 0.26 & 0.27 & 0.02 \\ 0.00 & 0.00 & 0.00 & 0.00 & 0.00 & 0.00 & 0.00 & 0.00 & 0.00 \\ 89.66 & 91.25 & 90.81 & 89.75 & 90.14 & 91.54 & 89.15 & 83.47 & 89.12\end{array}$

\begin{tabular}{rrrrrrrrr}
7.53 & 6.98 & 7.02 & 7.16 & 7.19 & 7.562 & 7.400 & 7.235 & 7.378 \\
0.04 & 0.06 & 0.05 & 0.01 & 0.02 & 0.052 & 0.023 & 0.006 & 0.028 \\
0.50 & 1.34 & 1.32 & 1.05 & 1.02 & 1.098 & 0.758 & 1.086 & 0.778 \\
2.24 & 2.49 & 2.53 & 2.19 & 2.14 & 1.395 & 1.613 & 1.904 & 1.659 \\
0.01 & 0.00 & 0.00 & 0.00 & 0.00 & 0.000 & 0.009 & 0.000 & 0.000 \\
3.60 & 2.87 & 2.77 & 3.51 & 3.53 & 3.299 & 3.999 & 3.461 & 3.980 \\
0.11 & 0.37 & 0.37 & 0.27 & 0.27 & 0.258 & 0.263 & 0.304 & 0.287 \\
0.16 & 0.12 & 0.15 & 0.06 & 0.05 & 0.080 & 0.085 & 0.191 & 0.083 \\
0.11 & 0.20 & 0.20 & 0.17 & 0.19 & 0.171 & 0.102 & 0.175 & 0.099 \\
0.01 & 0.00 & 0.02 & 0.00 & 0.00 & 0.006 & 0.006 & 0.001 & 0.001 \\
0.01 & 0.03 & 0.01 & 0.01 & 0.02 & 0.037 & 0.030 & 0.035 & 0.002 \\
22.00 & 22.00 & 22.00 & 22.000 & 22.000 & 22.000 & 22.000 & 22.000 & 22.000 \\
14.29 & 14.45 & 14.44 & 14.42 & 14.40 & 13.96 & 14.29 & 14.40 & 14.30 \\
\hline
\end{tabular}

Stead, David Emley, and David Kelsall, Geology Department, University of Keele, are gratefully acknowledged for their consummate skill in linking disparate computing systems and maintaining a sense of humor. Thanks are also due to the reviewers who tightened up the presentation of the analytical data.

\section{REFERENCES}

Adamson, A. C., 1981. Chemistry of alteration minerals from Deep Sea Drilling Project Sites 501, 504, and 505. In Cann, J. R., Langseth, M. G., Honnorez, J., Von Herzen, R. P., White, S. M., et al., Init. Repts. DSDP, 69: Washington (U.S. Govt. Printing Office), 551-563.
Allen, J. F., Sack, R. O., and Batiza, R., 1988. Cr-rich spinels as petrogenetic indicators: MORB-type lavas from the Lamont seamount chain, eastern Pacific. Am. Mineral., 73:741-753.

Alt, J. C., Laverne, C., and Muehlenbachs, K., 1985. Alteration of the upper oceanic crust: mineralogy and processes in DSDP Hole 504B, Leg 83. In Anderson, R. N., Honnorez, J., Becker, K., et al., Init. Repts. DSDP, 83: Washington (U.S. Govt. Printing Office), 217-247.

Bence, A. E., Papike, J. J., and Ayuso, R. A., 1975. Petrology of submarine basalts from the central Caribbean: DSDP Leg 15. J. Geophys. Res., 80:4775-4804.

Curtis, L. W., and Gittins, J., 1979. Aluminous and titaniferous clinopyroxenes from regionally metamorphosed agpaitic rocks in central Labrador. $J$. Petrol., 20:165-186. 
Deer, W. A., Howie, R. A., and Zussman, J., 1978. Rock Forming Minerals (Vol. 2A): Single Chain Silicates: London (Longman).

Droop, G.T.R., 1987. A general equation for estimating $\mathrm{Fe}^{3+}$ concentrations in ferromagnesian silicates and oxides from microprobe analysis, using stoichiometric criteria. Mineral. Mag., 51:431-437.

Ferguson, A. K., 1977. The natural occurrence of aegirine-neptunite solid solution. Contrib. Mineral. Petrol., 60:247-253.

Fisk, M. R., and Bence, A. E., 1980. Experimental crystallization of chrome spinel in FAMOUS basalt 527-1-1. Earth Planet. Sci. Lett., 48:111-123.

Flower, M.J.F., 1974. Phase relations of titanacmite in the system $\mathrm{Na}_{2} \mathrm{O}$ $\mathrm{Fe}_{2} \mathrm{O}_{3}-\mathrm{Al}_{2} \mathrm{O}_{3}-\mathrm{TiO}_{2}-\mathrm{SiO}_{2}$ at 1000 bars total water pressure. Am. Mineral., 59:536-548.

Floyd, P. A., 1989. Geochemical features of intraplate oceanic plateau basalts. In Saunders, A. D., and Norry, M. J. (Eds.), Magmatism in the Ocean Basins. Geol. Soc. Spec. Publ. London, 42:215-230.

Floyd, P. A., Castillo, P. R., and Pringle, M., 1991. Tholeiitic and alkali basalts of the oldest Pacific ocean crust. Terra Nova, 3:257-265.

Floyd, P. A., and Rowbotham, R., 1986. Chemistry of primary and secondary phases in intraplate basalts and volcaniclastic sediments, Deep Sea Drilling Project Leg 89. In Moberly, R., Schlanger, S. O., et al., Init. Repts. DSDP, 89: Washington (U.S. Govt. Printing Office), 459-470.

Furuta, T, and Tokuyama, H., 1983. Chromium spinels in Costa Rica basalts, Deep Sea Drilling Project Site 505-a preliminary interpretation of electron microprobe analyses. In Cann, J. R., Langseth, M. G., Honnorez, J., Von Herzen, R. P., White, S. M., et al., Init. Repts, DSDP, 69: Washington (U.S. Govt. Printing Office), 805-810.

Guven, N., 1988. Smectites. In Bailey, S. W. (Ed.), Hydrous Phyllosilicates. Mineral. Soc. Am., Rev. Mineral., 19:497-550.

Hawthorne, F. C., 1981. The crystal chemistry of amphiboles. In Veblen, D. R. and Ribbe, P. H. (Eds.), Amphiboles and Other Hydrous Pyriboles. Mineral. Soc. Am., Rev. Mineral., 9A:1-102.

Helz, R. T., 1973. Phase relations of basalts in their melting at $\mathrm{P}_{\mathrm{H}_{2} \mathrm{O}}=5 \mathrm{~Kb}$. Part II: Melt compositions. J. Petrol., 17:139-193.

, 1980. Crystallization history of Kilauea Iki lava lake as seen in drill core recovered in 1967-1979. Bull. Volcanol., 43:675-701.

Henderson, C.M.B., and Gibb, F.G.F., 1983. Felsic mineral crystallization trends in differentiating alkaline basic magmas. Contrib. Mineral. Petrol., $84: 355-364$

1988. The petrology of the Lugar Sill, S.W. Scotland. Trans. R. Soc. Edinburgh, 77:325-347.

Kempton, P. D., Autio, L. K., Rhodes, J. M., Holdaway, M. J., Dungan, M. A., and Johnson, P., 1985. Petrology of basalts from Hole 504B, Deep Sea Drilling Project, Leg 83. In Anderson, R. N., Honnorez, J., Becker, K., et al., Init. Repts. DSDP, 83: Washington (U.S. Govt. Printing Office), $165-183$.

Lancelot, Y., Larson, R. L., et al., 1990. Proc. ODP, Init. Repts., 129: College Station, TX (Ocean Drilling Program).
Laverne, C., 1987. Unusual occurrences of aegirine-augite, fassaite and melanite in oceanic basalts (DSDP 504B). Lithos, 20:135-151.

Leake, B. E., 1978. Nomenclature on amphiboles (for subcommittee on amphiboles, IMA). Mineral. Mag., 42:533-563.

Longhi, J., Walker, D., and Hayes, J. F., 1978. The distribution of Fe and Mg between olivine and lunar basaltic liquids. Geochim. Cosmochim. Acta, 42:1545-1558.

Lovering, J. F., and White, A.J.R., 1969. High-Al fassaites in eclogite inclusions of nephelinite pipes in New South Wales, Australia. Contrib. Mineral. Petrol., 21:9-52.

Matsuoka, A., 1990. The oldest (Middle Jurassic) radiolarians from the western Pacific. Proc. 3rd Int. Symp. "Shallow Tethys," Tokyo, Japan.

Morimoto, N., 1988. Nomenclature of pyroxenes (Commission on new minerals and mineral names, IMA). Am. Mineral., 73:1123-1133.

Roberts, J. L., 1976. Ti solubility in synthetic phlogopite solid solutions. Chem. Geol., 17:213-227.

Robinson, P., 1980. The composition space of terrestrial pyroxenes - internal and external limits. In Prewitt, C. T. (Ed.), Pyroxenes. Mineral. Soc. Am. Rev. Mineral., 7:419-494.

Roeder, P. L., 1974. Activity of iron and olivine solubility in basaltic liquids Earth Planet. Sci. Lett., 23:397-410.

Sack, R. O., and Carmichael, I.S.E., 1984. $\mathrm{Fe}=\mathrm{Mg}$ and $\mathrm{TiAl}_{2}=\mathrm{MgSi}_{2}$ exchange reactions between clinopyroxenes and silicate melts. Contrib. Mineral. Petrol., 85:103-115.

Schmincke, H. U., 1969. Ignimbritic sequences of Gran Canaria. Bull. Volcanol., 33:1199-1218.

Schumacher, J. C., 1991. Empirical ferric iron corrections: necessity assumptions, and effects on selected geothermobarometers. Mineral. Mag., $55: 3-18$.

Sigurdsson, H., and Schilling, J.-G., 1976. Spinels in Mid-Atlantic Ridge basalts: chemistry and occurrence. Earth Planet. Sci. Lett., 20:7-20.

Tracy, R. J., and Robinson, P., 1977. Zoned titanian augite in alkali olivine basalt from Tahiti and the nature of titanium substitution in augite. Am. Mineral. 62:634-645.

Upton, B.G.J., Stephenson, D., and Martin, A. R., 1985. The Tugtutoq older giant dyke complex: mineralogy and geochemistry of an alkali gabbro-augite syenite-foyaite association in the Gardar Province of south Greenland. Mineral. Mag., 49:623-642.

Velde, B., 1985. Clay Minerals: A Physico-chemical Explanation of Their Occurrence: Amsterdam (Elsevier), Dev. in Sedimentol. Ser., 40.

Wilkinson, J.F.G., 1956. Clinopyroxenes of alkali basalt magma. Am. Mineral. 41:724-743.

Date of initial receipt: 3 June 1991

Date of acceptance: 2 March 1992

Ms 129B-127 


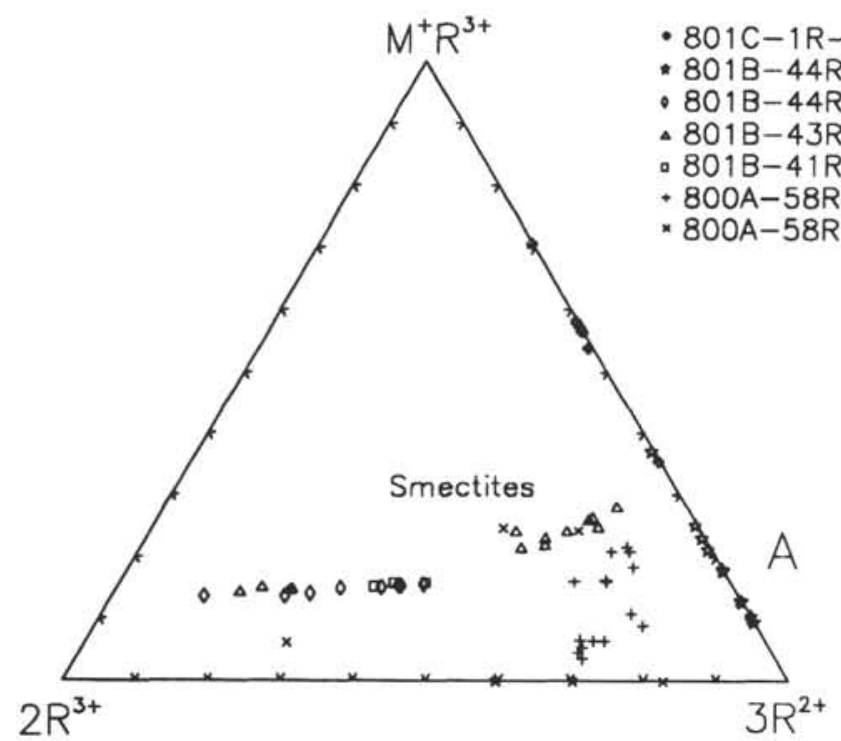

$14-20 \mathrm{~cm}$

$801 \mathrm{~B}-44 \mathrm{R}-3,28-33 \mathrm{~cm}$

- $801 \mathrm{~B}-44 \mathrm{R}-2,140-145 \mathrm{~cm}$

. $801 \mathrm{~B}-43 \mathrm{R}-1,4-9 \mathrm{~cm}$

$801 \mathrm{~B}-41 \mathrm{R}-1,130-136 \mathrm{~cm}$

(8) $-58 \mathrm{R}-2,67-73 \mathrm{~cm}$

$800 A-58 R-1,86-91 \mathrm{~cm}$
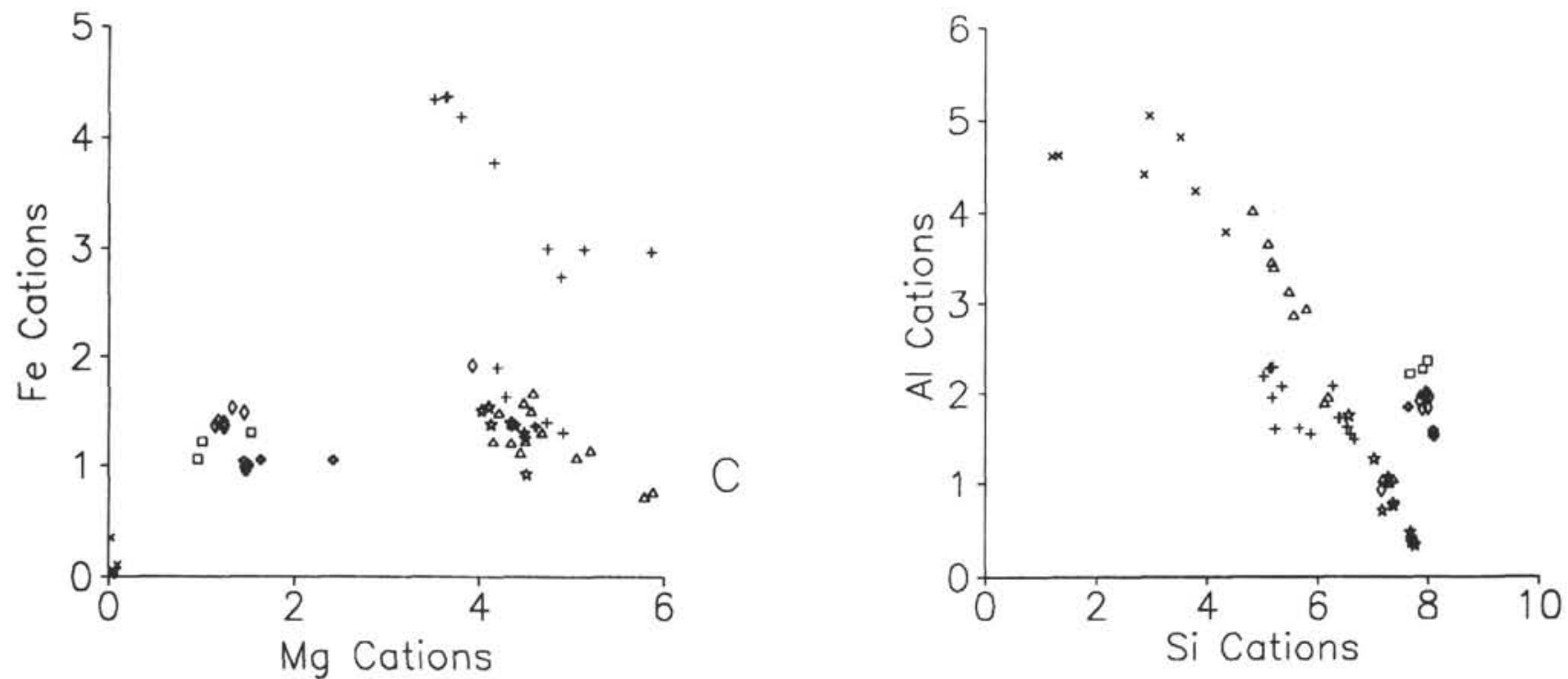

Figure 13. Composition of phyllosilicates from alkalic dolerite sills and basalt lava flows, Holes $800 \mathrm{~A}, 801 \mathrm{~B}$, and $801 \mathrm{C} . \mathrm{A} \cdot \mathrm{M}^{+} \mathrm{R}^{3+}-3 \mathrm{R}^{2+}-2 \mathrm{R}^{3+}$ components (modified after Velde, 1985). B. Interlayer cation variation. C. Fe vs. Mg contents. D. Total Al vs. Si contents. 
Table 14. Celadonite analyses from Leg 129 dolerites and basalts.

\begin{tabular}{|c|c|c|c|c|c|c|c|c|c|c|c|}
\hline Hole & $801 \mathrm{C}$ - & 801C - & $801 \mathrm{C}$ - & $801 C$ - & $801 C$ - & $801 \mathrm{C}-$ & $801 C-$ & $801 \mathrm{C}-$ & $801 C-$ & $801 \mathrm{C}-$ & $802 \mathrm{~A}-$ \\
\hline Core/section & $2 R-5$ & $2 R-5$ & $2 R-5$ & $2 R-5$ & $5 R-2$ & $5 R-2$ & $5 R-2$ & $5 R-2$ & $5 R-5$ & 5R-5. & $58 R-3$ \\
\hline Interval $(\mathrm{cm})$ & $101-106$ & $101-106$ & $101-106$ & $101-106$ & $123-130$ & $123-130$ & $123-130$ & $123-130$ & $73-78$ & $73-78$ & $90-95$ \\
\hline
\end{tabular}

\begin{tabular}{|c|c|c|c|c|c|c|c|c|c|c|c|}
\hline $\mathrm{SiO}_{2}$ & 58.01 & 57.78 & 58.32 & 57.51 & 57.11 & 56.76 & 55.42 & 56.53 & 57.61 & 55.53 & 53.05 \\
\hline $\mathrm{TiO}_{2}$ & 0.11 & 0.15 & 0.12 & 0.05 & 0.01 & 0.14 & 0.13 & 0.12 & 0.12 & 0.02 & 0.00 \\
\hline $\mathrm{Al}_{2} \mathrm{O}_{3}$ & 9.89 & 9.72 & 9.55 & 9.33 & 7.17 & 8.62 & 6.08 & 6.60 & 2.98 & 3.64 & 2.73 \\
\hline $\mathrm{FeO}$ & 7.97 & 8.21 & 8.74 & 8.53 & 16.65 & 14.81 & 17.95 & 18.06 & 19.15 & 19.81 & 16.92 \\
\hline Mno & 0.05 & 0.00 & 0.00 & 0.00 & 0.00 & 0.18 & 0.00 & 0.00 & 0.00 & 0.00 & 0.00 \\
\hline $\mathrm{MgO}$ & 7.03 & 7.12 & 7.28 & 7.14 & 5.58 & 5.99 & 5.22 & 4.98 & 5.07 & 5.21 & 9.06 \\
\hline $\mathrm{CaO}$ & 0.05 & 0.26 & 0.19 & 0.17 & 0.59 & 0.47 & 0.71 & 0.80 & 0.45 & 0.43 & 0.54 \\
\hline $\mathrm{Na}_{2} \mathrm{O}$ & 0.33 & 0.25 & 0.15 & 0.11 & 0.23 & 0.14 & 0.25 & 0.02 & 0.17 & 0.14 & 0.25 \\
\hline $\mathrm{K}_{2} \mathrm{O}$ & 0.10 & 9.74 & 9.91 & 9.79 & 7.66 & 8.38 & 7.30 & 6.86 & 7.59 & 8.07 & 7.41 \\
\hline $\mathrm{Cr}_{2} \mathrm{O}_{3}$ & 9.61 & 0.08 & 0.12 & 0.09 & 0.00 & 0.00 & 0.23 & 0.00 & 0.05 & 0.00 & 0.03 \\
\hline NiO & 0.00 & 0.00 & 0.51 & 0.40 & 0.26 & 0.22 & 0.40 & 0.00 & 0.25 & 0.43 & 0.00 \\
\hline 0 & 0.00 & 0.00 & 0.00 & 0.00 & 0.00 & 0.00 & 0.00 & 0.00 & 0.00 & 0.00 & 0.00 \\
\hline TOTAL \% & 93.09 & 93.30 & 94.87 & 93.12 & 95.30 & 95.70 & 93.68 & 93.97 & 93.45 & 93.27 & 89.98 \\
\hline
\end{tabular}

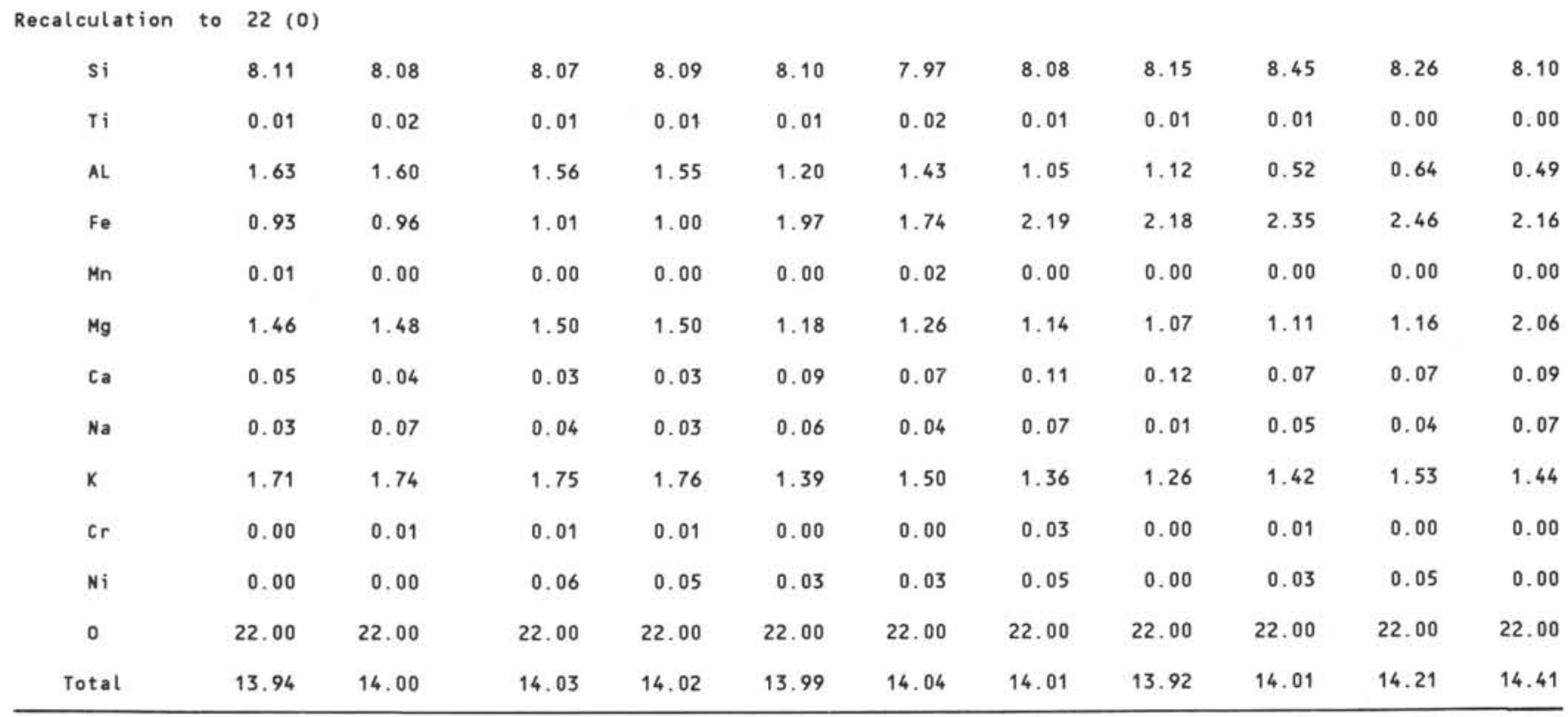

All celadonites are interstitial. 
Table 14 (continued).

\begin{tabular}{rrrrrrrrr}
\hline $802 A-$ & $802 A-$ & $802 A-$ & $802 A-$ & $802 A-$ & $802 A-$ & $802 A-$ & $802 A-$ & $802 A-$ \\
$58 R-3$. & $58 R-3$. & $58 R-3$. & $59 R-1$. & $59 R-1$. & $59 R-3$. & $59 R-3$. & $59 R-3$. & $59 R-3$. \\
$90-95$ & $90-95$ & $90-95$ & $136-142$ & $136-142$ & $40-44$ & $40-44$ & $40-44$ & $40-44$ \\
\hline & & & & & & & & \\
& & & & & & & & \\
51.85 & 53.33 & 54.31 & 51.79 & 51.78 & 52.18 & 52.15 & 50.33 & 51.37 \\
0.12 & 0.29 & 0.00 & 0.09 & 0.13 & 0.09 & 0.10 & 0.06 & 0.10 \\
1.13 & 2.44 & 2.25 & 2.46 & 2.70 & 3.26 & 2.32 & 2.08 & 1.82 \\
17.83 & 16.57 & 17.48 & 25.47 & 24.61 & 22.83 & 25.39 & 25.42 & 25.78 \\
0.00 & 0.00 & 0.05 & 0.03 & 0.00 & 0.01 & 0.00 & 0.00 & 0.00 \\
6.34 & 6.17 & 6.35 & 3.50 & 3.45 & 6.33 & 3.63 & 3.75 & 3.39 \\
3.99 & 0.00 & 0.00 & 0.64 & 0.58 & 0.89 & 0.52 & 0.40 & 0.56 \\
0.15 & 0.27 & 0.23 & 0.27 & 0.15 & 0.20 & 0.37 & 0.39 & 0.31 \\
9.47 & 9.87 & 10.13 & 7.06 & 6.96 & 5.88 & 7.27 & 7.12 & 7.52 \\
0.07 & 0.04 & 0.00 & 0.00 & 0.00 & 0.00 & 0.17 & 0.09 & 0.02 \\
0.00 & 0.00 & 0.00 & 0.70 & 0.20 & 0.00 & 0.00 & 0.00 & 0.00 \\
0.00 & 0.00 & 0.00 & 0.00 & 0.00 & 0.00 & 0.00 & 0.00 & 0.00 \\
90.93 & 88.99 & 90.80 & 92.00 & 90.55 & 91.66 & 91.94 & 89.65 & 90.87
\end{tabular}

\begin{tabular}{rrrrrrrrr}
8.10 & 8.32 & 8.33 & 8.10 & 8.16 & 7.99 & 8.14 & 8.10 & 8.16 \\
0.01 & 0.03 & 0.00 & 0.01 & 0.02 & 0.01 & 0.01 & 0.01 & 0.01 \\
0.21 & 0.45 & 0.41 & 0.45 & 0.50 & 0.59 & 0.43 & 0.39 & 0.34 \\
2.33 & 2.16 & 2.24 & 3.33 & 3.24 & 2.92 & 3.31 & 3.42 & 3.43 \\
0.00 & 0.00 & 0.01 & 0.00 & 0.00 & 0.00 & 0.00 & 0.00 & 0.00 \\
1.48 & 1.43 & 1.45 & 0.82 & 0.81 & 1.45 & 0.85 & 0.90 & 0.80 \\
0.67 & 0.00 & 0.00 & 0.11 & 0.10 & 0.15 & 0.09 & 0.07 & 0.10 \\
0.05 & 0.08 & 0.07 & 0.08 & 0.05 & 0.06 & 0.11 & 0.12 & 0.10 \\
1.89 & 1.96 & 1.98 & 1.41 & 1.40 & 1.15 & 1.45 & 1.46 & 1.53 \\
0.01 & 0.01 & 0.00 & 0.00 & 0.00 & 0.00 & 0.02 & 0.01 & 0.00 \\
0.00 & 0.00 & 0.00 & 0.09 & 0.03 & 0.00 & 0.00 & 0.00 & 0.00 \\
22.00 & 22.00 & 22.00 & 22.00 & 22.00 & 22.00 & 22.00 & 22.00 & 22.00 \\
14.74 & 14.45 & 14.49 & 14.41 & 14.30 & 14.31 & 14.41 & 14.48 & 14.46 \\
\hline
\end{tabular}



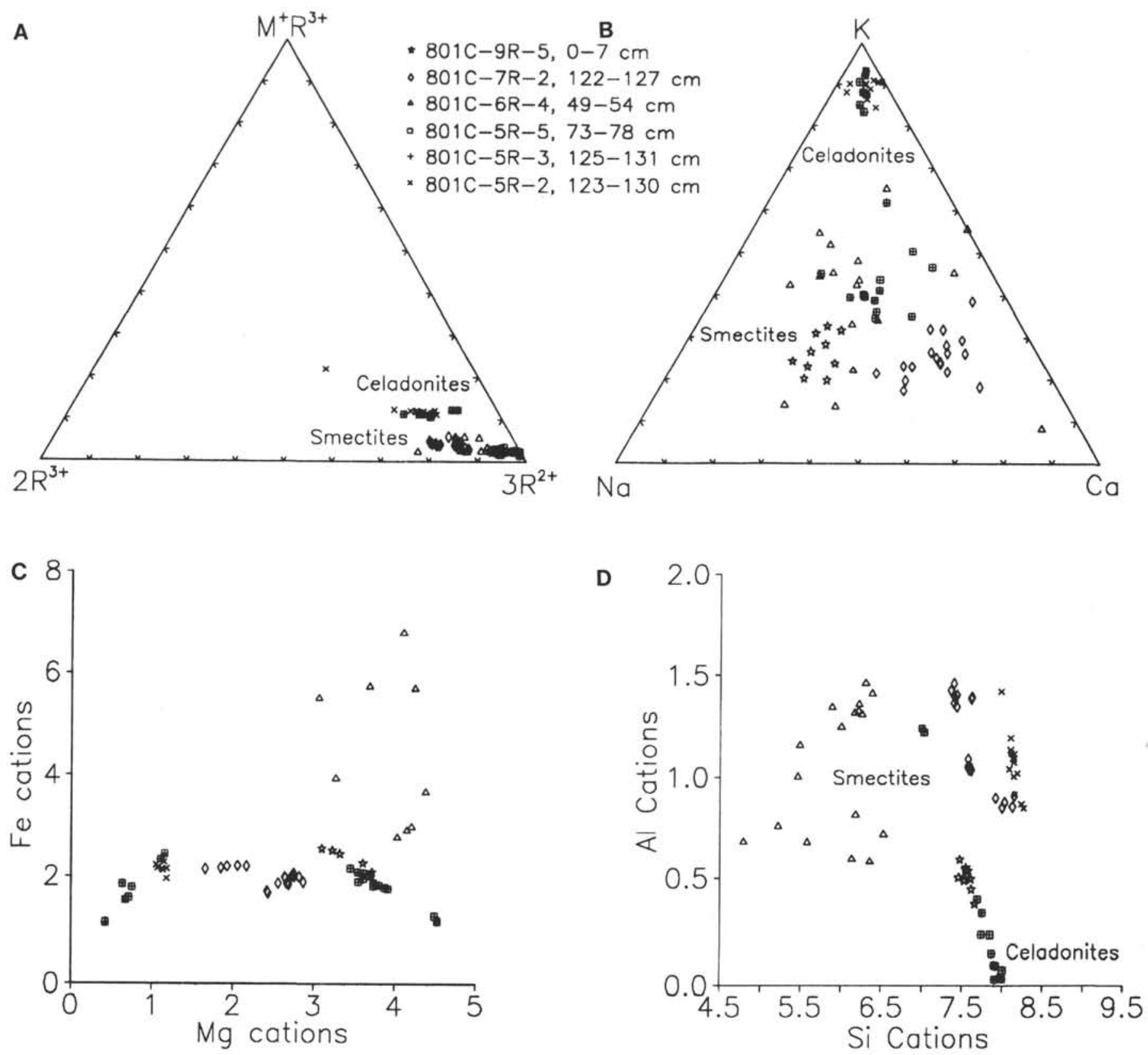

Figure 14. Composition of phyllosilicates from tholeiitic basalt flows, Hole $801 \mathrm{C} . \mathrm{A} . \mathrm{M}^{+} \mathrm{R}^{3+}-3 \mathrm{R}^{2+}-2 \mathrm{R}^{3+}$ components (modified after Velde, 1985). B. Interlayer cation variation. C. Fe vs. Mg contents. D. Total Al vs. Si contents.

Table 15. Analyses of carbonates from a traverse across the infill of a fracture in Sample 129-801C-5R-3, 125-131 cm.

\begin{tabular}{cccccc}
\hline Zone & Position & Ca carbonate & Mg carbonate & Fe carbonate & Mn carbonate \\
\hline 1 & \multirow{2}{*}{ Margin } & 94.6 & 1.8 & 2.8 & 0.9 \\
2 & & 17.5 & 21.7 & 52.7 & 8.0 \\
3 & & 60.4 & 21.8 & 16.4 & 1.4 \\
4 & Center & 95.2 & 1.6 & 2.4 & 0.7 \\
5 & & 18.1 & 19.2 & 55.8 & 7.0 \\
6 & Margin & 60.1 & 21.2 & 17.3 & 1.4 \\
\hline
\end{tabular}



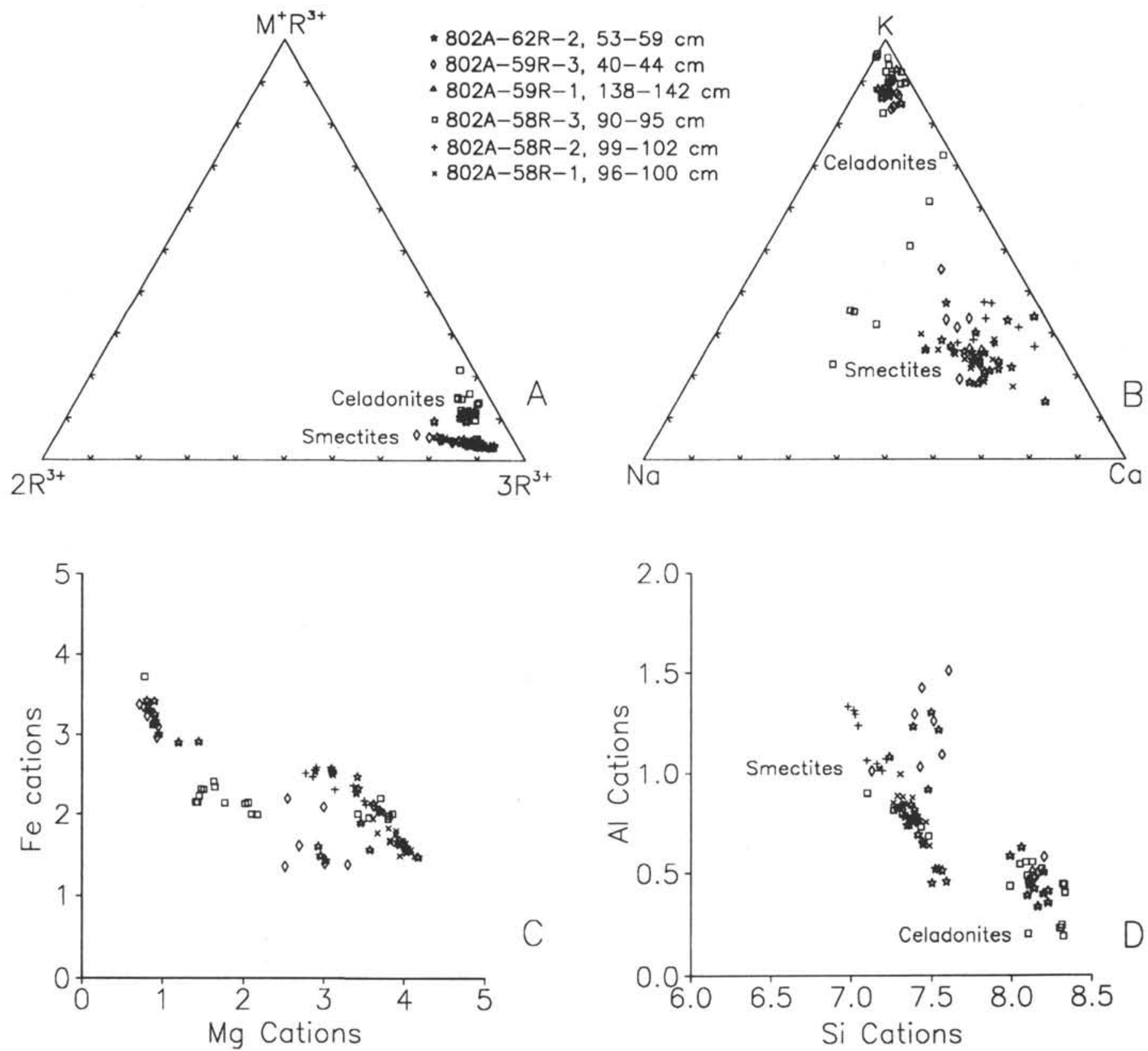

Figure 15. Composition of phyllosilicates from tholeiitic basalt flows, Hole 802A. A. $M^{+} R^{3+}-3 R^{2+}-2 R^{3+}$ components (modified after Velde, 1985). B. Interlayer cation variation. C. Fe vs. Mg contents. D. Total Al vs. Si contents. 